\title{
Boundary triplets, tensor products and point contacts to reservoirs
}

\author{
A.A. Boitsev J.F. Brasche M.M. Malamud \\ H. Neidhardt I.Yu. Popov
}

July 26, 2021

\begin{abstract}
We consider symmetric operators of the form $S:=A \otimes I_{\mathfrak{T}}+I_{\mathfrak{H}} \otimes T$ where $A$ is symmetric and $T=T^{*}$ is (in general) unbounded. Such operators naturally arise in problems of simulating point contacts to reservoirs. We construct a boundary triplet $\Pi_{S}$ for $S^{*}$ preserving the tensor structure. The corresponding $\gamma$-field and Weyl function are expressed by means of the $\gamma$-field and Weyl function corresponding to the boundary triplet $\Pi_{A}$ for $A^{*}$ and the spectral measure of $T$. Applications to 1-D Schrödinger and Dirac operators are given. A model of electron transport through a quantum dot assisted by cavity photons is proposed. In this model the boundary operator is chosen to be the well-known Jaynes-Cumming operator which is regarded as the Hamiltonian of the quantum dot.
\end{abstract}

Mathematics Subject Classification: 47A80, 47B25, 81Q05, 81Q37

Keywords: point contacts, reservoirs, symmetric operators, self-adjoint extensions, boundary triplet, Weyl function, quantum transport 


\section{Contents}

1 Introduction $\quad 2$

2 Preliminaries 7

2.1 Linear relations . . . . . . . . . . . . . . . . . . . . 7

2.2 Boundary triplets and proper extensions . . . . . . . . . . 7

$2.3 \gamma$-field and Weyl function . . . . . . . . . . . . . . 9

2.4 Krein-type formula for resolvents . . . . . . . . . . . . . . . 10

2.5 Normalized boundary triplets . . . . . . . . . . . . . . . 11

3 Operator-spectral integrals 14

4 Boundary triplets for tensor products 19

4.1 Bounded case . . . . . . . . . . . . . . . . 19

4.2 Unbounded case . . . . . . . . . . . . . . . . . . . . . 26

5 Sums of tensor products with non-negative summands 35

5.1 Boundary triplets in the case of non-negative operators $A$ and $T \quad 35$

5.2 Friedrichs and Krein extensions of $S:=A \otimes I_{\mathfrak{T}}+I_{\mathfrak{H}} \otimes T$. . . . 40

6 Examples 44

6.1 Schrödinger operators and bosons in $1 \mathrm{D} \ldots \ldots \ldots \ldots$

6.1.1 Schrödinger operators on half-lines . . . . . . . . . . . 44

6.1.2 Schrödinger operators on bounded intervals . . . . . . . . 46

6.2 Dirac operators and bosons in 1D . . . . . . . . . . . . 48

6.2.1 Dirac operators on half-lines . . . . . . . . . . . 48

6.2.2 Dirac operators on bounded intervals . . . . . . . . . 50

$7 \quad$ A model for electronic transport through a boson cavity 52

\section{Introduction}

In the following we are interested in the description of point contacts of quantum systems to quantum reservoirs. Let us recall the general philosophy of modeling of point contacts in quantum mechanics. Let $\left\{\mathfrak{K}, S_{0}\right\}$ be a quantum system where $S_{0}$ is a self-adjoint operator acting on the separable Hilbert space $\mathfrak{K}$. To describe point interactions one restricts the self-adjoint operator $S_{0}$ to a densely defined closed symmetric operator $S$ and extends it subsequently to another self-adjoint operator $S^{\prime}$. The new self-adjoint operator $S^{\prime}$ is regarded as the Hamiltonian taking into account point interactions. Which extension one has to choose depends on the physical problem. Typical examples for instance are $\delta$ and $\delta^{\prime}$-point interactions, cf. [5]. From the mathematical point of view it is interesting to note that the problem of describing point interactions fits into the framework of extension theory for symmetric operators. 
To describe point contacts of a quantum system with a reservoir one has to specify the approach. At first, one considers the compound system consisting of the quantum system $\left\{\mathfrak{H}, A_{0}\right\}$ and the reservoir $\{\mathfrak{T}, T\}$ where $A_{0}$ and $T$ are self-adjoint operators on the separable Hilbert spaces $\mathfrak{H}$ and $\mathfrak{T}$, respectively. Its Hamiltonian is given by the self-adjoint operator

$$
S_{0}:=A_{0} \otimes I_{\mathfrak{T}}+I_{\mathfrak{H}} \otimes T
$$

where $S_{0}$ acts in the Hilbert space $\mathfrak{K}:=\mathfrak{H} \otimes \mathfrak{T}$. To model a contact to the quantum reservoir, the Hamiltonian $S_{0}$ is usually additively perturbed in a suitable manner, cf. [22, 24, 23]. On the other hand, to model point contacts to reservoirs we use the restricting-extension procedure: We restrict the operator $A_{0}=A_{0}^{*}$ to a densely defined closed symmetric operator $A$ and consider the closed symmetric operator

$$
S:=A \otimes I_{\mathfrak{T}}+I_{\mathfrak{H}} \otimes T \subset S_{0} .
$$

From the physical point of view, the restriction of $A_{0}$ to $A$ and the subsequent extension to a self-adjoint operator $S^{\prime}$ can be regarded as the opening of the quantum system $\left\{\mathfrak{H}, A_{0}\right\}$ and the subsequent coupling of it to a reservoir. The self-adjoint extension $S^{\prime}$ should be different from $S_{0}$. However, self-adjoint extensions preserving tensor product form $\widetilde{S}=\widetilde{A} \otimes I_{\mathfrak{T}}+I_{\mathfrak{H}} \otimes T$ with $\widetilde{A}=\widetilde{A}^{*}$ being an extension of $A$, do not describe any interaction with the reservoir. From the physical point of view it is very important to describe all those extensions, which really describe point interactions with the reservoir.

In this paper we investigate operator (1.2) in the framework of boundary triplets and the corresponding Weyl functions. This is a new approach to the extension theory of symmetric operators that has been developed during the last three decades (see e.g. 25, 29, 28, 27, 32, 39, 58,).

A boundary triplet $\Pi_{A}=\left\{\mathcal{H}^{A}, \Gamma_{0}^{A}, \Gamma_{1}^{A}\right\}$ for the adjoint operator $A^{*}$ of a densely defined closed symmetric operator $A$ consists of an auxiliary Hilbert space $\mathcal{H}^{A}$ and linear mappings $\Gamma_{0}^{A}, \Gamma_{1}^{A}: \operatorname{dom}\left(A^{*}\right) \longrightarrow \mathcal{H}^{A}$ such that the abstract Green's identity

$$
\left(A^{*} f, g\right)-\left(f, A^{*} g\right)=\left(\Gamma_{1}^{A} f, \Gamma_{0}^{A} g\right)-\left(\Gamma_{0}^{A} f, \Gamma_{1}^{A} g\right), \quad f, g \in \operatorname{dom}\left(A^{*}\right),
$$

holds and the mapping

$$
\Gamma^{A}:=\left(\begin{array}{c}
\Gamma_{0}^{A} \\
\Gamma_{1}^{A}
\end{array}\right): \operatorname{dom}\left(A^{*}\right) \longrightarrow \stackrel{\mathcal{H}^{A}}{\oplus} \underset{\mathcal{H}^{A}}{\oplus}
$$

is surjective.

A boundary triplet for $A^{*}$ exists whenever $A$ has equal deficiency indices. It plays the role of a "coordinate system" for the quotient space $\operatorname{dom}\left(A^{*}\right) / \operatorname{dom}(A)$ and leads to a natural parametrization of the self-adjoint extensions of $A$ by means of self-adjoint linear relations (multi-valued operators) in $\mathcal{H}$, see 32 and 
58 for details. More precisely, any self-adjoint extension $\widetilde{A}$ of $A$ defines a selfadjoint relation $\Theta:=\Gamma^{A} \operatorname{dom}(\widetilde{A})$ in $\mathcal{H}^{A}$ and vice versa. We write $A_{\Theta}=\widetilde{A}$, i.e.

$$
\operatorname{dom}(\widetilde{A})=\operatorname{dom}\left(A_{\Theta}\right):=\left\{f \in \operatorname{dom}\left(A^{*}\right): \Gamma^{A} f \in \Theta\right\} .
$$

If $\Theta$ is an operator $\Theta=B$, this relation takes the form

$$
\operatorname{dom}(\widetilde{A})=\operatorname{dom}\left(A_{B}\right):=\left\{f \in \operatorname{dom}\left(A^{*}\right): \Gamma_{1}^{A} f=B \Gamma_{0}^{A} f\right\}
$$

and looks like an abstract boundary condition. Among all self-adjoint extensions there are two augmented ones: $A_{0}:=A^{*} \uparrow \operatorname{ker}\left(\Gamma_{0}^{A}\right)$ and $A_{1}:=A^{*} \uparrow \operatorname{ker}\left(\Gamma_{1}^{A}\right)$ which correspond to self-adjoint relations $\Theta_{0}:=(0, f)^{t}$ and $\Theta_{1}:=(f, 0)^{t}, f \in \mathcal{H}$, respectively. Clearly, $\Theta_{1}^{-1}=\Theta_{0}$.

The main analytical tool in this approach is the abstract Weyl function $M^{A}(\cdot)$ which was introduced and studied in 228,29 . This abstract Weyl function $M^{A}(\cdot)$ plays a similar role in the theory of boundary triplets as the classical Weyl-Titchmarsh function does it in the theory of Sturm-Liouville operators. In particular, it allows one to investigate spectral properties of extensions (see [15, 28, 47, 49]). The Weyl function is defined by

$$
M^{A}(z):=\Gamma_{1}^{A} \gamma^{A}(z), \quad z \in \rho\left(A_{0}\right)
$$

where

$$
\gamma^{A}(z):=\left(\Gamma_{0}\left\lceil\mathfrak{N}_{z}\right)^{-1} \quad, \quad \mathfrak{N}_{z}:=\operatorname{ker}\left(A^{*}-z\right), \quad z \in \rho\left(A_{0}\right) .\right.
$$

Here $\gamma^{A}(\cdot)$ is the so-called $\gamma$-field, the second important quantity related to a boundary triplet $\Pi_{A}$.

Emphasize that a boundary triplet for $A^{*}$ is not unique. Its role in extension theory is similar to that of a coordinate system in analytic geometry. The problem is to construct an adequate ("good") boundary triplet such that the corresponding Weyl function and the boundary operator corresponding to the extension of interest have "good" properties. To demonstrate the later point we mention boundary triplets for direct sum of maximal 1-D Schrödinger operators $-d^{2} / x^{2}+q$ in $L^{2}\left[x_{n-1}, x_{n}\right]$ constructed in [42, 43, 20, where the boundary operator corresponding to 1-D Schrödinger operator with infinitely many point interactions in $L^{2}\left(\mathbb{R}_{+}\right)=\bigoplus_{n=1}^{\infty} L^{2}\left[x_{n-1}, x_{n}\right]$ is a Jacobi matrix.

This approach has successfully been applied to the characterization of the absolutely continuous spectrum of self-adjoint realizations [15, 49, as well as to the investigation of the spectral properties of 1-D Schrödinger and 1-D Dirac operators with point interactions [30, 42, 40, 41, 43, 20, 3-D Schrödinger operators with point interactions [51, and elliptic boundary value problems in domains with compact boundaries [33, 34, 35], [16, 17, 48, 8, 9, to the scattering theory [10, 11], etc. Especially we mention the works [31, 32, 50] (see also the literature quoted therein) where the Sturm-Liouville operator with unbounded operator potential was treated as an operator admitting the tensor structure (1.2). 
Our goal in this paper is to apply the boundary triplet approach to the problem of coupling of a quantum system to a reservoir by point interactions. More precisely, the mathematically rigorous problem is: Given a boundary triplet $\Pi_{A}=\left\{\mathcal{H}^{A}, \Gamma_{0}^{A}, \Gamma_{1}^{A}\right\}$ for $A^{*}$ one should construct an adequate ("good") boundary triplet $\Pi_{S}$ for $S^{*}$ with $S$ given by (1.2) and such that

$$
S_{0}:=S^{*} \uparrow \operatorname{ker}\left(\Gamma_{0}^{S}\right)=A_{0} \otimes I_{\mathfrak{T}}+I_{\mathfrak{H}} \otimes T
$$

and compute the corresponding Weyl function and $\gamma$-field.

So starting with a given boundary triplet $\Pi_{A}$ for $A^{*}$ a "good" candidate for a boundary triplet $\Pi_{S}:=\left\{\mathcal{H}^{S}, \Gamma_{0}^{S}, \Gamma^{S}\right\}$ for $S^{*}$ would be

$$
\mathcal{H}^{S}=\mathcal{H}^{A} \otimes \mathfrak{T}, \quad \Gamma_{0}^{S}:=\Gamma_{0}^{A} \otimes I_{\mathfrak{T}} \quad \Gamma_{1}^{S}:=\Gamma_{1}^{A} \otimes I_{\mathfrak{T}} .
$$

This triplet feels the tensor structure of the problem. For instance, according to (1.3) and (1.4) an extension $S^{\prime}=A^{\prime} \otimes I_{\mathfrak{T}}+I_{\mathfrak{H}} \otimes T \in$ Ext $_{\mathrm{S}}$ admits a representation $S^{\prime}=S_{B_{S}^{\prime}}$ with the boundary operator $B_{S}^{\prime}=\left(B_{S}^{\prime}\right)^{*}$ having the tensor form, $B_{S}^{\prime}=B_{A}^{\prime} \otimes I_{\mathfrak{T}}$, where $B_{A}^{\prime}=\left(B_{A}^{\prime}\right)^{*}$ is the boundary operator of $A^{\prime}=A^{\prime *} \in \operatorname{Ext}_{\mathrm{A}}$ in the triplet $\Pi_{A}$, i.e. $A^{\prime}=A_{B_{A}^{\prime}}$. In particular, we have $S_{0}=A_{0} \otimes I_{\mathfrak{T}}+I_{\mathfrak{H}} \otimes T$. Hence any extension $S_{B^{\prime}}=S_{B^{\prime}}^{*} \in$ Ext $_{\mathrm{S}}$ with the boundary operator $B^{\prime}$ not admitting tensor structure, can be regarded as a Hamiltonian describing a point interaction with a reservoir.

It is shown in [14, 50] that the triplet (1.4) is a boundary triplet for $S^{*}$ whenever $T$ is bounded. However this fails for unbounded $T$, a case naturally arising in physical problems. This case requires new ideas and is much more technically involved.

Let $\Pi_{A}=\left\{\mathcal{H}^{A}, \Gamma_{0}^{A}, \Gamma_{1}^{A}\right\}$ be a boundary triplet for $A^{*}$ and let $M^{A}(\cdot)$ and $\gamma^{A}(\cdot)$ be the corresponding Weyl function and $\gamma$-field, respectively. Using the regularization procedure introduced and developed in [50, 42, 20] we construct a special boundary triplet $\Pi_{S}=\left\{\mathcal{H}^{S}, \Gamma_{0}^{S}, \Gamma_{1}^{S}\right\}$ for $S^{*}$ such that $S_{0}:=S^{*} \uparrow$ $\operatorname{ker}\left(\Gamma_{0}^{S}\right)=A_{0} \otimes I_{\mathfrak{T}}+I_{\mathfrak{H}} \otimes T$. Moreover, we show in Theorem 4.8 that the corresponding $\gamma$-field $\gamma^{S}(\cdot)$ and Weyl function $M^{S}(\cdot)$ are given by

$$
\gamma^{S}(z) f:=\int_{\mathbb{R}}\left(\gamma^{A}(z-\lambda) \frac{1}{\sqrt{\operatorname{Im}\left(M^{A}(i-\lambda)\right)}} \otimes I_{\mathfrak{T}}\right) \widehat{E}_{T}(d \lambda) f,
$$

and

$$
M^{S}(z) f:=\int_{\mathbb{R}}\left(L^{A}(z-\lambda, i-\lambda) \otimes I_{\mathfrak{T}}\right) \widehat{E}_{T}(d \lambda) f \quad z \in \mathbb{C}_{ \pm} .
$$

where

$$
L^{A}(z, \zeta):=\frac{1}{\sqrt{\operatorname{Im}\left(M^{A}(\zeta)\right)}}\left(M^{A}(z)-\operatorname{Re}\left(M^{A}(\zeta)\right)\right) \frac{1}{\sqrt{\operatorname{Im}\left(M^{A}(\zeta)\right)}},
$$

$z \in \mathbb{C}_{ \pm}, \zeta \in \mathbb{C}_{+}$, and $\widehat{E}_{T}(\cdot):=I_{\mathcal{H}^{A}} \otimes E_{T}(\cdot)$, where $E_{T}(\cdot)$ is the spectral measure of $T=T^{*}$. Here both improper integrals exist for every $f \in \mathcal{H}^{A} \otimes \mathfrak{T}$. 
We apply formula (1.6) for $M^{S}(\cdot)$ to show that for non-negative $A \geq 0$ and $T \geq 0$ the Friedrichs and Krein extensions of $S \geq 0$ are given by

$$
\widehat{S}_{F}=\widehat{A}_{F} \otimes I_{\mathfrak{T}}+I_{\mathfrak{H}} \otimes T, \quad \widehat{S}_{K}=\widehat{A}_{K} \otimes I_{\mathfrak{T}}+I_{\mathfrak{H}} \otimes T,
$$

where $\widehat{A}_{F}$ and $\widehat{A}_{K}$ are the Friedrichs and Krein extensions of $A$, respectively. In turn, we apply these formulas to show that if $T \in \mathcal{B}(\mathfrak{T})$, then the operator $S$ has LSB-property (each semibounded boundary operator $B$ defines a semibounded extension $S_{B}$ of $S$ ) if and only if the operator $A \geq 0$ has the LSB-property.

This approach can be used to propose a model describing rigorously the electron transport through a quantum dot assisted by photons, a topic which is of great interests for physicists, cf. [1, 36, 44, 57, etc. In this case we start from the operator (1.1) with $A_{0}$ being Sturm-Liouville operator on the line with piece-wise constant potential and unbounded $T$ given by $T=b^{*} b$, where $b^{*}$ and $b$ are the creation and annihilation operators, respectively. We define $A$ as a restriction of $A_{0}$ to the domain $\operatorname{dom}(A)=W_{0}^{2,2}\left(\mathbb{R}_{-}\right) \oplus W_{0}^{2,2}\left(\mathbb{R}_{+}\right)$and then define the operator $S$ by (1.2). We construct a boundary triplet for $S^{*}$ feeling the tensor structure (1.2), and compute the Weyl function (a special case of (1.6) ).

An interesting feature of our approach is to define Hamiltonian describing the point contact to the reservoir by means of a boundary operator. To this end we choose the boundary operator to be well-known Jaynes-Cumming operator (see [37])

$$
C_{J C}=B \otimes I_{\mathfrak{T}}+I_{\mathcal{H}^{A}} \otimes T+\tau V_{J C}, \quad \tau \in \mathbb{R},
$$

The operator $C_{J C}$ has a physical meaning and is regarded as the Hamiltonian of the quantum dot. From the mathematical point of view it is interesting to note that the Jaynes-Cumming Hamiltonian is in fact a Jacobi matrix.

In this connection we mention the papers by Pavlov [54, 55, 56] treating several solvable physical models in the framework of extension theory.

In a forthcoming paper we plan to express the scattering matrix for a naturally related scattering system by means of the Weyl function, using results from [10, 11. Explicit knowledge of the scattering matrix allows to calculate the current going through the quantum dot using the so-called Landauer-Büttiker formula invented in [19, 46, see also [6, 21] for a mathematically rigorous proof of this formula. Using this approach our final goal is to compute explicitly the electron and photon current going through the quantum dot of the proposed model.

The paper is organized as follows. In Section 2 we give a short introduction into the boundary triplet approach. In particular, we consider the case of a direct sum of symmetric operators. Section 3 is devoted to operator-spectral integrals needed in the following. In Section 4 we consider boundary triplets for tensor products. First we compute explicitly the Weyl function and $\gamma$-field for the triplet (1.4) with a bounded $T \in \mathcal{B}(\mathfrak{H})$ by using the functional calculus developed in Section 3. In Section 4.2 we construct an adequate boundary triplet for $S^{*}$ assuming $T$ to be unbounded and prove formulas (1.6) and (1.5). Section 5 is devoted to the case of non-negative operators $A$ and $T$, a situation typical in 
physics. In particular, formulas (1.7) are proved here. In Section 6 we illustrate the abstract results of Sections 4,5, by typical physical examples. In particular, we consider Schrödinger and Dirac operators and a reservoir of bosons on halfline and bounded intervals. Finally, in Section 7 we use the previous examples to propose a simple model describing a photon assisted electronic transport through a quantum dot.

Notation. Let $\mathfrak{H}_{1}, \mathfrak{H}_{2}$ be separable Hilbert spaces. By $\mathcal{C}\left(\mathfrak{H}_{1}, \mathfrak{H}_{2}\right)$ $\left(\mathcal{B}\left(\mathfrak{H}_{1}, \mathfrak{H}_{2}\right)\right)$ we denote the set of closed (bounded) linear operators from $\mathfrak{H}_{1}$ to $\mathfrak{H}_{2} ; \mathcal{C}(\mathfrak{H}):=\mathcal{C}(\mathfrak{H}, \mathfrak{H}), \mathcal{B}(\mathfrak{H}):=\mathcal{B}(\mathfrak{H}, \mathfrak{H})$. By $\mathfrak{S}_{p}(\mathfrak{H}), p \in(0, \infty]$, we denote the Schatten-Neumann ideals of order $p$ on $\mathfrak{H}$; in particular, $\mathfrak{S}_{\infty}(\mathfrak{H})$ is the ideal of compact operators on $\mathfrak{H}$. By $\operatorname{dom}(T), \operatorname{ran}(T), \rho(T)$ and $\sigma(T)$ we denote the domain, range resolvent set and spectrum of the operator $T$, respectively.

\section{Preliminaries}

\subsection{Linear relations}

A linear relation $\Theta$ in $\mathcal{H}$ is a closed linear subspace of $\mathcal{H} \oplus \mathcal{H}$. The set of all linear relations in $\mathcal{H}$ is denoted by $\widetilde{\mathcal{C}}(\mathcal{H})$. Denote also by $\mathcal{C}(\mathcal{H})$ the set of all closed linear (not necessarily densely defined) operators in $\mathcal{H}$. Identifying each operator $T \in \mathcal{C}(\mathcal{H})$ with its graph $\operatorname{gr}(T)$ we regard $\mathcal{C}(\mathcal{H})$ as a subset of $\widetilde{\mathcal{C}}(\mathcal{H})$.

The role of the set $\widetilde{\mathcal{C}}(\mathcal{H})$ in extension theory becomes clear from Proposition 2.3. However, it's role in the operator theory is substantially motivated by the following circumstances: in contrast to $\mathcal{C}(\mathcal{H})$, the set $\widetilde{\mathcal{C}}(\mathcal{H})$ is closed with respect to taking inverse and adjoint relations $\Theta^{-1}$ and $\Theta^{*}$. Here $\Theta^{-1}=\{\{g, f\}$ : $\{f, g\} \in \Theta\}$ and

$$
\Theta^{*}=\left\{\left(\begin{array}{c}
k \\
k^{\prime}
\end{array}\right):\left(h^{\prime}, k\right)=\left(h, k^{\prime}\right) \text { for all }\left(\begin{array}{c}
h \\
h^{\prime}
\end{array}\right) \in \Theta\right\} .
$$

A linear relation $\Theta$ is called symmetric if $\Theta \subset \Theta^{*}$ and self-adjoint if $\Theta=\Theta^{*}$.

\subsection{Boundary triplets and proper extensions}

Following [32] and [28] we briefly recall some basic facts on boundary triplets. Let $S$ be a densely defined closed symmetric operator in a separable Hilbert space $\mathfrak{H}$ and let $n_{ \pm}(S):=\operatorname{dim}\left(\mathfrak{N}_{ \pm \mathrm{i}}\right)$ be its deficiency indices, where $\mathfrak{N}_{z}:=$ $\operatorname{ker}\left(S^{*}-z\right), z \in \mathbb{C}_{ \pm}$, is the defect subspace of $S$.

Definition 2.1 A closed extension $\widetilde{S}$ of $S$ is called proper if $\operatorname{dom}(S) \varsubsetneqq$ $\operatorname{dom}(\widetilde{S}) \varsubsetneqq \operatorname{dom}\left(S^{*}\right)$.

We denote by Ext S $_{\mathrm{S}}$ the set of all proper extensions of $S$ completed by the nonproper extensions $S$ and $S^{*}$. It is known that any dissipative (accumulative), in particular symmetric, extension of $S$ is proper. 
Definition 2.2 (cf. [32]) A triplet $\Pi_{S}=\left\{\mathcal{H}^{S}, \Gamma_{0}^{S}, \Gamma_{1}^{S}\right\}$, where $\mathcal{H}^{S}$ is an auxiliary Hilbert space and $\Gamma_{0}^{S}, \Gamma_{1}^{S}: \operatorname{dom}\left(S^{*}\right) \rightarrow \mathcal{H}^{S}$ are linear mappings, is called a boundary triplet for $S^{*}$ if the 'abstract Green's identity'

$$
\left(S^{*} f, g\right)-\left(f, S^{*} g\right)=\left(\Gamma_{1}^{S} f, \Gamma_{0}^{S} g\right)-\left(\Gamma_{0}^{S} f, \Gamma_{1}^{S} g\right), \quad f, g \in \operatorname{dom}\left(S^{*}\right),
$$

holds and the mapping $\Gamma^{S}:=\left(\Gamma_{0}^{S}, \Gamma_{1}^{S}\right)^{t}: \operatorname{dom}\left(S^{*}\right) \rightarrow\left(\mathcal{H}^{S} \oplus \mathcal{H}^{S}\right)^{t}$ is surjective, i.e. $\operatorname{ran}\left(\Gamma^{S}\right)=\left(\mathcal{H}^{S} \oplus \mathcal{H}^{S}\right)^{t}$.

A boundary triplet $\Pi_{S}=\left\{\mathcal{H}^{S}, \Gamma_{0}^{S}, \Gamma_{1}^{S}\right\}$ for $S^{*}$ always exists whenever $n_{+}(S)=$ $n_{-}(S)$. Note also that $n_{ \pm}(S)=\operatorname{dim}\left(\mathcal{H}^{\mathrm{S}}\right)$ and $\operatorname{ker}\left(\Gamma_{0}^{S}\right) \cap \operatorname{ker}\left(\Gamma_{1}^{S}\right)=\operatorname{dom}(S)$.

The linear maps $\Gamma_{j}^{S}: \operatorname{dom}\left(S^{*}\right) \longrightarrow \mathcal{H}^{S}, j=0,1$, are neither bounded nor closable. However, equipping the domain $\operatorname{dom}\left(S^{*}\right)$ with the graph norm

$$
\|f\|_{S^{*}}^{2}:=\left\|S^{*} f\right\|^{2}+\|f\|^{2}, \quad f \in \operatorname{dom}\left(S^{*}\right),
$$

one obtains a Hilbert space $\mathfrak{H}_{+}\left(S^{*}\right)$. and regarding

It turns out that the mappings $\Gamma_{j}^{S}: \operatorname{dom}\left(S^{*}\right) \longrightarrow \mathcal{H}^{S}, j \in\{0,1\}$, as the mappings from $\mathfrak{H}_{+}\left(S^{*}\right)$ into $\mathcal{H}^{S}$ are already bounded.

In what follows we denote by $\widehat{\Gamma}_{j}^{S}$ the operator $\Gamma_{j}^{S}$ treated as the mapping $\widehat{\Gamma}_{j}^{S}: \mathfrak{H}_{+}\left(S^{*}\right) \longrightarrow \mathcal{H}^{S}, j \in\{0,1\}$. If $J_{S^{*}}: \mathfrak{H}_{+}\left(S^{*}\right) \longrightarrow \operatorname{dom}\left(S^{*}\right)$ denotes the embedding operator, then $\widehat{\Gamma}_{j}^{S}=\Gamma_{j}^{S} J_{S^{*}}, j \in\{0,1\}$. It follows from Definition 2.2 that $\operatorname{ran}\left(\widehat{\Gamma}^{S}\right)=\mathcal{H}^{S} \oplus \mathcal{H}^{S}$, where $\widehat{\Gamma}^{S}:=\left(\widehat{\Gamma}_{0}^{S}, \widehat{\Gamma}_{1}^{S}\right)^{t}$. Notice that the abstract Green's identity (2.1) can be written as

$$
\left(S^{*} J_{S^{*}} f, J_{S^{*}} g\right)-\left(J_{S^{*}} f, S^{*} J_{S^{*}} g\right)=\left(\widehat{\Gamma}_{1}^{S} f, \widehat{\Gamma}_{0}^{S} g\right)-\left(\widehat{\Gamma}_{0}^{S} f, \widehat{\Gamma}_{1}^{S} g\right),
$$

$f, g \in \mathfrak{H}_{+}\left(S^{*}\right)$. With any boundary triplet $\Pi_{S}$ one associates two canonical selfadjoint extensions $S_{j}:=S^{*} \uparrow \operatorname{ker}\left(\Gamma_{j}^{S}\right), j \in\{0,1\}$. Conversely, for any extension $S_{0}=S_{0}^{*} \in$ Ext $_{S}$ there exists a (non-unique) boundary triplet $\Pi_{S}=\left\{\mathcal{H}^{S}, \Gamma_{0}^{S}, \Gamma_{1}^{S}\right\}$ for $S^{*}$ such that $S_{0}:=S^{*} \uparrow \operatorname{ker}\left(\Gamma_{0}^{S}\right)$.

Using the concept of boundary triplets one can parametrize all proper extensions of $A$ in the following way.

Proposition 2.3 (cf. [28, 47]) Let $\Pi_{S}=\left\{\mathcal{H}^{S}, \Gamma_{0}^{S}, \Gamma_{1}^{S}\right\}$ be a boundary triplet for $S^{*}$. Then the mapping

$$
\operatorname{Ext}_{\mathrm{S}} \ni \widetilde{\mathrm{S}} \rightarrow \Gamma^{\mathrm{S}} \operatorname{dom}(\widetilde{\mathrm{S}})=\left\{\left(\Gamma_{0}^{\mathrm{S}} \mathrm{f}, \Gamma_{1}^{\mathrm{S}} \mathrm{f}\right)^{\mathrm{t}}: \mathrm{f} \in \operatorname{dom}(\widetilde{\mathrm{S}})\right\}=: \Theta \in \widetilde{\mathcal{C}}\left(\mathcal{H}^{\mathrm{S}}\right)
$$

establishes a bijective correspondence between the sets $\operatorname{Ext}_{S}$ and $\widetilde{\mathcal{C}}\left(\mathcal{H}^{S}\right)$. We write $\widetilde{S}=S_{\Theta}$ if $\widetilde{S}$ corresponds to $\Theta$ by (2.2). Moreover, the following holds:

(i) $S_{\Theta}^{*}=S_{\Theta^{*}}$, in particular, $S_{\Theta}^{*}=S_{\Theta}$ if and only if $\Theta^{*}=\Theta$.

(ii) $S_{\Theta}$ is symmetric (self-adjoint) if and only if so is $\Theta$. 
In particular, $S_{j}:=S^{*} \uparrow \operatorname{ker}\left(\Gamma_{j}^{S}\right)=S_{\Theta_{j}}, j \in\{0,1\}$, where $\Theta_{0}:=\left(\begin{array}{l}\{0\} \\ \mathcal{H}^{S}\end{array}\right)$ and $\Theta_{1}:=\left(\begin{array}{l}\mathcal{H}^{S} \\ \{0\}\end{array}\right)=\operatorname{gr}(\mathbb{O})$ where $\mathbb{O}$ denotes the zero operator in $\mathcal{H}^{S}$. Note also that the trivial linear relations $\{0\} \times\{0\}$ and $\mathcal{H}^{S} \times \mathcal{H}^{S} \in \widetilde{\mathcal{C}}\left(\mathcal{H}^{S}\right)$ parametrize the extensions $S$ and $S^{*}$, respectively, in any triplet $\Pi_{S}$.

\section{$2.3 \gamma$-field and Weyl function}

It is well known that the Weyl function is an important tool in the direct and inverse spectral theory of Sturm-Liouville operators. In [29, 28, the concept of Weyl function was generalized to the case of an arbitrary symmetric operator $S$ with $n_{+}(S)=n_{-}(S) \leq \infty$. Following [28, we briefly recall basic facts on Weyl functions and $\gamma$-fields, associated with a boundary triplet $\Pi$. For further properties and applications see [18, 28, 27], [58] (and references therein).

Definition 2.4 (cf. [29, 28]) Let $\Pi^{S}=\left\{\mathcal{H}^{S}, \Gamma_{0}^{S}, \Gamma_{1}^{S}\right\}$ be a boundary triplet for $S^{*}$ and $S_{0}=S^{*} \uparrow \operatorname{ker}\left(\Gamma_{0}^{S}\right)$. The operator valued functions $\gamma^{S}(\cdot): \rho\left(S_{0}\right) \rightarrow$ $\mathcal{B}\left(\mathcal{H}^{S}, \mathfrak{H}\right)$ and $M^{S}(\cdot): \rho\left(S_{0}\right) \rightarrow \mathcal{B}\left(\mathcal{H}^{S}\right)$ defined by

$$
\gamma^{S}(z):=\left(\Gamma_{0}^{S} \uparrow \mathfrak{N}_{z}\right)^{-1} \quad \text { and } \quad M^{S}(z):=\Gamma_{1}^{S} \gamma^{S}(z), \quad z \in \rho\left(S_{0}\right),
$$

are called the $\gamma$-field and the Weyl function, respectively, corresponding to the boundary triplet $\Pi_{S}$.

Clearly, the Weyl function can equivalently be defined by

$$
M^{S}(z) \Gamma_{0}^{S} f_{z}=\Gamma_{1}^{S} f_{z}, \quad f_{z} \in \mathfrak{N}_{z}, \quad z \in \rho\left(S_{0}\right) .
$$

The $\gamma$-field $\gamma^{S}(\cdot)$ and the Weyl function $M^{S}(\cdot)$ in (2.3) are well defined. Moreover, both $\gamma^{S}(\cdot)$ and $M^{S}(\cdot)$ are holomorphic on $\rho\left(S_{0}\right)$ and satisfy the following relations

$$
\gamma^{S}(z)=\left(I+(z-\zeta)\left(S_{0}-z\right)^{-1}\right) \gamma^{S}(\zeta), \quad z, \zeta \in \rho\left(S_{0}\right),
$$

and

$$
M^{S}(z)-M^{S}(\zeta)^{*}=(z-\bar{\zeta}) \gamma^{S}(\zeta)^{*} \gamma^{S}(z), \quad z, \zeta \in \rho\left(S_{0}\right),
$$

hold. Identity (2.5) yields that $M^{S}(\cdot)$ is an $\mathcal{B}\left(\mathcal{H}^{S}\right)$-valued Nevanlinna function $\left(M^{S}(\cdot) \in R\left[\mathcal{H}^{S}\right]\right)$, i.e. $M^{S}(\cdot)$ is an $\mathcal{B}\left(\mathcal{H}^{S}\right)$-valued holomorphic function on $\mathbb{C}_{ \pm}$ satisfying

$$
M^{S}(z)=M^{S}(\bar{z})^{*} \quad \text { and } \quad \frac{\operatorname{Im}\left(M^{S}(z)\right)}{\operatorname{Im}(z)} \geq 0, \quad z \in \mathbb{C}_{ \pm} .
$$

It follows also from (2.5) that $0 \in \rho\left(\operatorname{Im}\left(M^{S}(z)\right)\right)$ for all $z \in \mathbb{C}_{ \pm}$.

Being an $R\left[\mathcal{H}^{S}\right]$-function the Weyl function $M^{S}(\cdot)$ admits an integral representation

$$
M^{S}(z)=C_{0}+\int_{\mathbb{R}}\left(\frac{1}{t-z}-\frac{t}{1+t^{2}}\right) d \Sigma_{S}(t), \quad \int_{\mathbb{R}} \frac{d \Sigma_{S}(t)}{1+t^{2}} \in \mathcal{B}\left(\mathcal{H}^{S}\right),
$$


where $C_{0}=C_{0}^{*}$ and $\Sigma_{S}(\cdot)$ is a left continuous $\left(\Sigma_{S}(t)=\Sigma_{S}(t-0)\right)$ monotone operator-valued function. Emphasize that the linear term $C_{1} z$ is missing in (2.6) because the operator $A$ is densely defined (see [28]).

A Weyl function $M^{S}(\cdot)$ is said to be of scalar type if there exists a scalar Nevanlinna function $m^{S}(\cdot)$ such that the representation

$$
M^{S}(z)=m^{S}(z) I_{\mathcal{H}^{S}}, \quad z \in \mathbb{C}_{ \pm}
$$

holds where $I_{\mathcal{H}^{S}}$ is the identity operator in $\mathcal{H}^{S}$, see [4]. Obviously, $M^{S}(\cdot)$ is of scalar type if $n_{ \pm}(A)=1$.

Next we extract from (2.6) lower and upper bounds for $\operatorname{Im}\left(M^{S}(i-\lambda)\right)$ which will be useful in the sequel. It follows from (2.6) that

$$
\operatorname{Im}\left(M^{S}(i-\lambda)\right)=\int_{\mathbb{R}} \frac{d \Sigma_{S}(t)}{(t-\lambda)^{2}+1}, \quad \lambda \in \mathbb{R}
$$

Note that with certain positive constants $C_{1}, C_{2}>0$ the following estimate holds

$$
\frac{C_{1}}{1+|\lambda|^{2}} \leq \frac{1+t^{2}}{(t-\lambda)^{2}+1} \leq C_{2}\left(1+|\lambda|^{2}\right), \quad \lambda \in \mathbb{R}
$$

Combining these estimates with the identity $\operatorname{Im} M(i)=\int_{\mathbb{R}}\left(1+t^{2}\right)^{-1} d \Sigma_{S}(t)$ one derives from (2.7) that

$$
C_{1}\left(1+|\lambda|^{2}\right)^{-1} \operatorname{Im} M(i) \leq \operatorname{Im} M^{S}(i-\lambda) \leq C_{2}\left(1+|\lambda|^{2}\right) \operatorname{Im} M(i), \quad \lambda \in \mathbb{R}
$$

Emphasize that since the proof of estimates (2.8) is based only on integral representation (2.6), these estimates are valid for any $R\left[\mathcal{H}^{S}\right]$-function not necessarily being a Weyl function.

\subsection{Krein-type formula for resolvents}

Let $\Pi_{S}=\left\{\mathcal{H}^{S}, \Gamma_{0}^{S}, \Gamma_{1}^{S}\right\}$ be a boundary triplet for $S^{*}$, and $M^{S}(\cdot)$ and $\gamma^{S}(\cdot)$ the corresponding Weyl function and $\gamma$-field, respectively. For any proper (not necessarily self-adjoint) extension $\widetilde{S}_{\Theta} \in \operatorname{Ext}_{S}$ with non-empty resolvent set $\rho\left(\widetilde{S}_{\Theta}\right)$ the following Krein-type formula holds (cf. [29, 28, 27])

$$
\left(S_{\Theta}-z\right)^{-1}-\left(S_{0}-z\right)^{-1}=\gamma^{S}(z)\left(\Theta-M^{S}(z)\right)^{-1}\left(\gamma^{S}(\bar{z})\right)^{*},
$$

$z \in \rho\left(S_{0}\right) \cap \rho\left(S_{\Theta}\right)$. Formula (2.9) extends the known Krein formula for canonical resolvents to the case of any $S_{\Theta} \in \operatorname{Ext}_{\mathrm{S}}$ with $\rho\left(S_{\Theta}\right) \neq \emptyset$. Moreover, due to relations (2.2) and (2.3) all objects in formula (2.9) are expressed by means of the boundary triplet $\Pi_{S}$. We emphasize, that this connection makes it possible to apply the Krein-type formula (2.9) to boundary value problems. 


\subsection{Normalized boundary triplets}

Let $S_{n}$ be a densely defined closed symmetric operator in $\mathfrak{H}_{n}, n \in \mathbb{Z}$, and let $S:=\bigoplus_{n \in \mathbb{Z}} S_{n}$. Clearly,

$$
\begin{aligned}
S^{*} & =\bigoplus_{n \in \mathbb{Z}} S_{n}^{*}, \\
\operatorname{dom}\left(S^{*}\right) & =\left\{f=\bigoplus_{\mathbb{Z}} f_{n} \in \mathfrak{H}: f_{n} \in \operatorname{dom}\left(S_{n}^{*}\right), \quad \sum_{n=1}^{\infty}\left\|S_{n}^{*} f_{n}\right\|^{2}<\infty\right\} .
\end{aligned}
$$

Let $\Pi_{S_{n}}=\left\{\mathcal{H}^{S_{n}}, \Gamma_{0}^{S_{n}}, \Gamma_{1}^{S_{n}}\right\}$ be a boundary triplet for $S_{n}^{*}, n \in \mathbb{Z}$. Define mappings $\Gamma_{j}^{S}, j \in\{0,1\}$, by setting

$$
\begin{aligned}
& \Gamma_{j}^{S}:=\bigoplus_{n \in \mathbb{Z}} \Gamma_{j}^{S_{n}}, \\
& \operatorname{dom}\left(\Gamma_{j}^{S}\right):=\left\{\bigoplus_{n \in \mathbb{Z}} f_{n} \in \operatorname{dom}\left(S^{*}\right): \sum_{n \in \mathbb{Z}}\left\|\Gamma_{j}^{S_{n}} f_{n}\right\|^{2}<\infty\right\} .
\end{aligned}
$$

Definition 2.5 Let $\Gamma_{j}^{S}$ be given by (2.10) and $\mathcal{H}^{S}:=\bigoplus_{n \in \mathbb{Z}} \mathcal{H}^{S_{n}}$. A collection $\Pi_{S}=\left\{\mathcal{H}^{S}, \Gamma_{0}^{S}, \Gamma_{1}^{S}\right\}$ is called a direct sum of boundary triplets and is assigned as $\Pi_{S}=\bigoplus_{n \in \mathbb{Z}} \Pi_{S_{n}}$.

It was first discovered by $A$. Kochubei 39] that the direct sum $\bigoplus \Pi_{n}$ of boundary triplets $\Pi_{n}$ is not a boundary triplet in general. Later on simple examples were constructed in [50], 42], 20]. Moreover, it was shown in [42, Theorem 3.2] that $\Pi_{S}$ is only a generalized boundary triplet (a boundary relation in the sense of [26]). Moreover, according to [25] $\Pi_{S}$ is a so called ES-generalized boundary triplet for $S^{*}$, since the operator $S_{0}:=S^{*} \uparrow \operatorname{ker}\left(\Gamma_{0}^{S}\right)$ is essentially selfadjoint.

The reason is that the domain $\operatorname{dom}\left(\Gamma_{j}^{S}\right), j \in\{0,1\}$, might be narrower than $\operatorname{dom}\left(S^{*}\right)$ and the range of the mapping $\Gamma^{S}:=\left(\Gamma_{0}^{S}, \Gamma_{1}^{S}\right)^{t}: \operatorname{dom}\left(S^{*}\right) \rightarrow$ $\left(\mathcal{H}^{S} \oplus \mathcal{H}^{S}\right)^{t}$ might be a proper subset of $\left(\mathcal{H}^{S} \oplus \mathcal{H}^{S}\right)^{t}$. Nevertheless, $\operatorname{dom}\left(\Gamma_{j}^{S}\right)$, $j \in\{0,1\}$, is always dense in $\mathfrak{H}_{+}\left(S^{*}\right)$ and its range ran $\left(\Gamma^{S}\right)$ is dense in $\left(\mathcal{H}^{S} \oplus\right.$ $\left.\mathcal{H}^{S}\right)^{t}$. Moreover, by 26. Proposition 5.3], $\Pi_{S}$ is a boundary triplet whenever $\operatorname{ran}\left(\Gamma^{S}\right)=\left(\mathcal{H}^{S} \oplus \mathcal{H}^{S}\right)^{t}$. Besides, in accordance with [42, Proposition 3.8] the conditions

$$
\sum_{n \in \mathbb{Z}}\left\|\Gamma_{j}^{S_{n}} f_{n}\right\|^{2}<\infty, \quad f=\bigoplus_{n \in \mathbb{Z}} f_{n} \in \operatorname{dom}\left(S^{*}\right), \quad j \in\{0,1\},
$$

imply that $\Pi_{S}=\bigoplus_{n \in \mathbb{Z}} \Pi_{S_{n}}$ is an ordinary boundary triplet, while the sole first condition in (2.11) (with $j=0$ ) ensures only that $\Pi_{S}$ is a $B$-generalized boundary triplet in the sense of [27], 25]. Moreover, according to [25] $\Pi_{S}$ is a so called ES-generalized boundary triplet for $S^{*}$, since the operator $S_{0}:=S^{*} \uparrow$ $\operatorname{ker}\left(\Gamma_{0}^{S}\right)$ is essentially self-adjoint. 
A regularization procedure described below was first proposed in [50] and has been applied to construct a boundary triplet for Sturm-Liouville operators

$$
-d^{2} / d x^{2} \otimes I_{\mathfrak{T}}+I_{\mathfrak{H}} \otimes T, \quad \mathfrak{H}=L^{2}\left(\mathbb{R}_{+} ; \mathfrak{T}\right)=L^{2}\left(\mathbb{R}_{+}\right) \otimes \mathfrak{T},
$$

with unbounded potential $T=T^{*} \in \mathcal{C}(\mathfrak{T})$. Further generalizations of regularization procedures as well as applications to Schrödinger and Dirac operators with $\delta$-interactions were obtained in [42] and [20, respectively.

Let $\Pi_{S}=\left\{\mathcal{H}^{S}, \Gamma_{0}^{S}, \Gamma_{1}^{S}\right\}$ be a boundary triplet for $S^{*}$ with Weyl function $M^{S}(\cdot)$. We call $\Pi_{S}$ a normalized boundary triplet for $S^{*}$ if the condition $M^{S}(i)=i I_{\mathcal{H}^{S}}$ is satisfied.

Lemma 2.6 ([50]) Let $\Pi_{S}=\left\{\mathcal{H}^{S}, \Gamma_{0}^{S}, \Gamma_{1}^{S}\right\}$ be a boundary triplet for $S^{*}$, let $\gamma^{S}(\cdot)$ and $M^{S}(\cdot)$ be the $\gamma(\cdot)$-field and Weyl function, respectively. Let $R_{S}:=$ $\sqrt{\operatorname{Im}\left(M^{S}(i)\right)}$ and $Q_{S}:=\operatorname{Re}\left(M^{S}(i)\right)$. Then $\widetilde{\Pi}_{S}=\left\{\widetilde{\mathcal{H}}^{S}, \widetilde{\Gamma}_{0}^{S}, \widetilde{\Gamma}_{1}^{S}\right\}$, where

$$
\widetilde{\mathcal{H}}^{S}:=\mathcal{H}^{S}, \quad \widetilde{\Gamma}_{0}^{S}:=R_{S} \Gamma_{0}^{S} \quad \text { and } \quad \widetilde{\Gamma}_{1}^{S}:=R_{S}^{-1}\left(\Gamma_{1}^{S}-Q_{S} \Gamma_{0}^{S}\right),
$$

is a normalized boundary triplet for $S^{*}$ such that

$$
S_{0}:=S^{*} \uparrow \operatorname{ker}\left(\Gamma_{0}^{S}\right)=S^{*} \uparrow \operatorname{ker}\left(\widetilde{\Gamma}_{0}^{S}\right) .
$$

The $\gamma$-field $\widetilde{\gamma}^{S}(\cdot)$ and Weyl function $\widetilde{M}^{S}(\cdot)$ corresponding to the triplet $\widetilde{\Pi}_{S}$ are given by

$$
\widetilde{\gamma}^{S}(z)=\gamma^{S}(z) R_{S}^{-1} \text { and } \widetilde{M}^{S}(z)=R_{S}^{-1}\left(M^{S}(z)-Q_{S}\right) R_{S}^{-1}, \quad z \in \mathbb{C}_{ \pm} .
$$

Lemma 2.6] shows that with any boundary triplet one can associate a normalized boundary triplet such that $S_{0}$ remains unchanged. The following theorem presents a regularization procedure for direct sums $\Pi_{S}=\bigoplus_{n \in \mathbb{Z}} \Pi_{S_{n}}$ to define an ordinary boundary triplet.

Theorem 2.7 (Theorem 3.3, [50]) Let $S_{n}$ be a densely defined closed symmetric operator in $\mathfrak{H}_{n}, n \in \mathbb{Z}$, and $S:=\oplus_{n \in \mathbb{Z}} S_{n}$. Let $\Pi_{S_{n}}=\left\{\mathcal{H}^{s_{n}}, \Gamma_{0}^{S_{n}}, \Gamma_{1}^{S_{n}}\right\}$ be a boundary triplet for $S_{n}^{*}, S_{0 n}:=S_{n}^{*} \uparrow \operatorname{ker}\left(\Gamma_{0}^{S_{n}}\right), n \in \mathbb{Z}$, and let $\gamma^{S_{n}}(\cdot)$ and $M^{S_{n}}(\cdot)$ be the corresponding $\gamma$-field and Weyl function, respectively. Finally, let $R_{S_{n}}:=\sqrt{\operatorname{Im}\left(M^{S_{n}}(i)\right)}$ and $Q_{S_{n}}:=\operatorname{Re}\left(M^{S_{n}}(i)\right), n \in \mathbb{Z}$. Then the triplet $\widetilde{\Pi}_{S}=\left\{\widetilde{\mathcal{H}}^{S}, \widetilde{\Gamma}_{0}^{S}, \widetilde{\Gamma}_{1}^{S}\right\}$ with

$$
\begin{aligned}
\widetilde{\mathcal{H}}^{S} & :=\bigoplus_{n \in \mathbb{N}} \mathcal{H}^{S_{n}}, \quad \widetilde{\Gamma}_{0}^{S}:=\bigoplus_{n \in \mathbb{Z}} R_{S_{n}} \Gamma_{0}^{S_{n}}, \\
\widetilde{\Gamma}_{1}^{S} & :=\bigoplus_{n \in \mathbb{Z}} R_{S_{n}}^{-1}\left(\Gamma_{1}^{S_{n}}-Q_{S_{n}} \Gamma_{0}^{S_{n}}\right),
\end{aligned}
$$

is a (normalized) boundary triplet for $S^{*}$ satisfying

$$
\widetilde{S}_{0}=S^{*} \uparrow \operatorname{ker}\left(\widetilde{\Gamma}_{0}^{S}\right)=\bigoplus_{n \in \mathbb{Z}} \widetilde{S}_{0 n}=\bigoplus_{n \in \mathbb{Z}} S_{0 n}, \quad \widetilde{S}_{0 n}=S_{n}^{*}\left\lceil\operatorname{ker}\left(\widetilde{\Gamma}_{0}^{S_{n}}\right) .\right.
$$


Moreover, the $\gamma$-field $\widetilde{\gamma}^{S}(\cdot)$ and Weyl function $\widetilde{M}^{S}(\cdot)$ corresponding to $\widetilde{\Pi}_{S}$ are given by

$$
\begin{aligned}
\widetilde{\gamma}^{S}(z) & =\bigoplus_{n \in \mathbb{Z}} \gamma^{S_{n}}(z) R_{S_{n}}^{-1} \quad \text { and } \\
\widetilde{M}^{S}(z) & =\bigoplus_{n \in \mathbb{Z}} R_{S_{n}}^{-1}\left(M^{S_{n}}(z)-Q_{S_{n}}\right) R_{S_{n}}^{-1}, \quad z \in \mathbb{C}_{ \pm} .
\end{aligned}
$$

Next we assume that the operator $S=\bigoplus_{n=1}^{\infty} S_{n}$ has a regular real point, i.e., there exists $a=\bar{a} \in \hat{\rho}(A)$. The latter is equivalent to the existence of $\varepsilon>0$ such that

$$
(a-\varepsilon, a+\varepsilon) \subset \cap_{n=1}^{\infty} \widehat{\rho}\left(S_{n}\right) .
$$

Emphasize that condition $a \in \cap_{n=1}^{\infty} \widehat{\rho}\left(S_{n}\right)$ is not sufficient for the inclusion $a \in \widehat{\rho}(A)$.

It is known (see e.g. [45, 2]) that under condition (2.15) for every $k \in \mathbb{N}$ there exists a self-adjoint extension $\widetilde{S}_{k}=\widetilde{S}_{k}^{*}$ of $S_{k}$ preserving the gap $(a-\varepsilon, a+\varepsilon)$. The latter amounts to saying that the Weyl function of the pair $\left\{S_{k}, \widetilde{S}_{k}\right\}$ is regular within the gap $(a-\varepsilon, a+\varepsilon)$.

For operators $S=\bigoplus_{n=1}^{\infty} S_{n}$ satisfying (2.15) we complete Theorem 2.7 by presenting a regularization procedure for $\Pi=\bigoplus_{n=1}^{\infty} \Pi_{n}$ leading to a boundary triplet (cf. [42, Theorem 3.13], [20, Theorem 2.12 and Corollary 2.13]). In applications to symmetric operators with a gap this regularization is more appropriate and simpler than the one described in Theorem 2.7.

Proposition $2.8([42,20])$ Let $\left\{S_{n}\right\}_{n=1}^{\infty}$ be a sequence of symmetric operators satisfying (2.15). Let also $\Pi_{n}=\left\{\mathcal{H}_{n}, \Gamma_{0}^{(n)}, \Gamma_{1}^{(n)}\right\}$ be a boundary triplet for $S_{n}^{*}$ such that $(a-\varepsilon, a+\varepsilon) \subset \rho\left(S_{0 n}\right), S_{0 n}=S_{n}^{*}\left\lceil\operatorname{ker}\left(\Gamma_{0}^{(n)}\right)\right.$. Let also $\gamma^{S_{n}}(\cdot)$ and $M_{n}(\cdot):=M^{S_{n}}(\cdot)$ be the corresponding $\gamma$-field and Weyl function, respectively. Assume also that for some operators $R_{n}$ such that $R_{n}, R_{n}^{-1} \in \mathcal{B}\left(\mathcal{H}_{n}\right)$, the following conditions are satisfied

$$
\begin{aligned}
& \sup _{n}\left\|R_{n}^{-1}\left(M_{n}^{\prime}(a)\right)\left(R_{n}^{-1}\right)^{*}\right\|_{\mathcal{H}_{n}}<\infty \quad \text { and } \\
& \sup _{n}\left\|R_{n}^{*}\left(M_{n}^{\prime}(a)\right)^{-1} R_{n}\right\|_{\mathcal{H}_{n}}<\infty, \quad n \in \mathbb{N} .
\end{aligned}
$$

Then the direct sum $\widetilde{\Pi}_{S}=\bigoplus_{n=1}^{\infty} \widetilde{\Pi}_{n}$ of boundary triplets where

$$
\begin{aligned}
\widetilde{\Pi}_{n} & =\left\{\mathcal{H}_{n}, \widetilde{\Gamma}_{0}^{(n)}, \widetilde{\Gamma}_{1}^{(n)}\right\}, \\
\widetilde{\Gamma}_{0}^{(n)} & :=R_{n} \Gamma_{0}^{(n)} \text { and } \widetilde{\Gamma}_{1}^{(n)}:=\left(R_{n}^{-1}\right)^{*}\left(\Gamma_{1}^{(n)}-M_{n}(a) \Gamma_{0}^{(n)}\right),
\end{aligned}
$$

forms a boundary triplet for $S^{*}=\bigoplus_{n=1}^{\infty} S_{n}^{*}$. by

Moreover, the corresponding $\gamma$-field $\widetilde{\gamma}^{S}(\cdot)$ and Weyl function $\widetilde{M}^{S}(\cdot)$ are given

$$
\begin{aligned}
\widetilde{\gamma}^{S}(z) & =\bigoplus_{n \in \mathbb{Z}} \gamma^{S_{n}}(z) R_{n}^{-1} \quad \text { and } \\
\widetilde{M}^{S}(z) & =\bigoplus_{n \in \mathbb{Z}}\left(R_{n}^{-1}\right)^{*}\left(M_{n}(z)-M_{n}(a)\right) R_{n}^{-1}, \quad z \in \mathbb{C}_{ \pm} .
\end{aligned}
$$


In particular one can set $R_{n}=\sqrt{M_{n}^{\prime}(a)}, n \in \mathbb{N}$.

Emphasize that $M_{n}^{\prime}(a)$ is a positive definite operator whenever $a \in \rho\left(S_{0 n}\right)$.

\section{Operator-spectral integrals}

Let $F(\cdot)$ be an orthogonal operator measure with compact support supp $(F) \subseteq$ $\Delta:=[a, b),-\infty<a<b<\infty$, and with values in $\mathcal{B}(\mathcal{H})$. Further, let $\Omega(\cdot)$ : $[a, b) \longrightarrow \mathcal{B}\left(\mathcal{H}, \mathcal{H}_{1}\right)$ be an operator-valued function. We consider partitions $\mathcal{Z}$ of $[a, b)$ of the form $[a, b)=\left[\lambda_{0}, \lambda_{1}\right) \cup\left[\lambda_{1}, \lambda_{2}\right) \cup \ldots \cup\left[\lambda_{n-1}, \lambda_{n}\right), \lambda_{0}=a, \lambda_{n}=b$ and set $\Delta_{m}:=\left[\lambda_{m-1}, \lambda_{m}\right), m=1,2, \ldots, n$. Thus $[a, b)=\bigcup_{m=1}^{n} \Delta_{m}$ and the $\Delta_{m}$ are pairwise disjoint. Let $|\mathcal{Z}|:=\max _{m=1,2, \ldots, n}\left|\Delta_{m}\right|$, where $\left|\Delta_{m}\right|:=\lambda_{m}-\lambda_{m-1}$. We define the operator $\Sigma_{\mathcal{Z}} \Omega$ by

$$
\Sigma_{\mathcal{Z}} \Omega=\sum_{m=1}^{n} \Omega\left(x_{m}\right) F\left(\Delta_{m}\right), \quad x_{m} \in \Delta_{m}
$$

The sum $\Sigma_{\mathcal{Z}} \Omega$ is called the Riemann-Stieltjes sum of $\Omega(\cdot)$ with respect to the operator measure $F(\cdot)$. If there is an operator $\Sigma_{0} \in \mathcal{B}\left(\mathcal{H}, \mathcal{H}_{1}\right)$ such that $\lim _{|\mathcal{Z}| \rightarrow 0}\left\|\Sigma_{\mathcal{Z}} \Omega-\Sigma_{0}\right\|=0$ independent of the special choice of $\mathcal{Z}$ and $\left\{x_{m}\right\}_{m=1}^{n}$, then $\Sigma_{0}$ is called the operator spectral integral of $\Omega(\cdot)$ with respect to $F(\cdot)$ and is denoted by

$$
\Sigma_{0}=: \int_{\Delta} \Omega(\lambda) F(d \lambda)
$$

Obviously, in a similar way one can define for operator-valued functions $\Omega$ : $\Delta \longrightarrow \mathcal{B}\left(\mathcal{H}_{1}, \mathcal{H}\right)$ the operator spectral integral $\int_{\Delta} F(d \lambda) \Omega(\lambda)$ as the limit of the Riemann-Stieltjes sums $\sum_{m} F\left(\Delta_{m}\right) \Omega\left(x_{m}\right)$. It is clear that the operator spectral integral is linear with respect to $\Omega(\cdot)$. If $B$ is a bounded operator, then

$$
B \int_{\Delta} \Omega(\lambda) F(d \lambda)=\int_{\Delta} B \Omega(\lambda) F(d \lambda)
$$

Definition 3.1 The operator-valued mapping $\Omega:[a, b) \longrightarrow \mathcal{B}(\mathcal{H})$ will be called $F$-admissible, if the integral $\int_{\Delta} \Omega(\lambda) F(d \lambda)$ exists and

$$
\Omega(\lambda) F(\delta)=F(\delta) \Omega(\lambda) F(\delta), \quad \delta \in \mathcal{B}([a, b)), \quad \lambda \in \Delta .
$$

Proposition 3.2 Let $\Omega:[a, b) \longrightarrow \mathcal{B}(\mathcal{H})$ be F-admissible, $\Omega_{1}:[a, b) \longrightarrow$ $\mathcal{B}\left(\mathcal{H}, \mathcal{H}_{1}\right)$, and assume that $\int_{\Delta} \Omega_{1}(\lambda) F(d \lambda)$ exists. Then $\int_{\Delta} \Omega_{1}(\lambda) \Omega(\lambda) F(d \lambda)$ exists and

$$
\int_{\Delta} \Omega_{1}(\lambda) \Omega(\lambda) F(d \lambda)=\int_{\Delta} \Omega_{1}(\delta) F(d \delta) \int_{\Delta} \Omega(\mu) F(d \mu) .
$$


Proof. It is easily seen that

$$
\Sigma_{\mathcal{Z}} \Omega_{1} \Sigma_{\mathcal{Z}} \Omega \longrightarrow \int_{\Delta} \Omega_{1}(\lambda) F(d \lambda) \int_{\Delta} \Omega(\mu) F(d \mu) \quad \text { as } \quad|\mathcal{Z}| \longrightarrow 0 .
$$

On the other hand, since the measure $F(\cdot)$ is orthogonal, $F\left(\Delta_{j}\right) F\left(\Delta_{k}\right)=$ $F\left(\Delta_{j}\right) \delta_{j k}, j, k \in\{1, \ldots, m\}$. Combining these relations with the $F$-admissibility of $\Omega$ yields

$$
\begin{aligned}
\Sigma_{\mathcal{Z}} \Omega_{1} \Sigma_{\mathcal{Z}} \Omega & =\sum_{m, m^{\prime}=1}^{n} \Omega_{1}\left(x_{m}\right) F\left(\Delta_{m}\right) \Omega\left(x_{m^{\prime}}\right) F\left(\Delta_{m^{\prime}}\right) \\
& =\sum_{m, m^{\prime}=1}^{n} \Omega_{1}\left(x_{m}\right) F\left(\Delta_{m}\right) F\left(\Delta_{m^{\prime}}\right) \Omega\left(x_{m^{\prime}}\right) F\left(\Delta_{m^{\prime}}\right) \\
& =\sum_{m=1}^{n} \Omega_{1}\left(x_{m}\right) F\left(\Delta_{m}\right) \Omega\left(x_{m}\right) F\left(\Delta_{m}\right) \\
& =\sum_{m=1}^{n} \Omega_{1}\left(x_{m}\right) \Omega\left(x_{m}\right) F\left(\Delta_{m}\right) \longrightarrow \int_{\Delta} \Omega_{1}(\lambda) \Omega(\lambda) F(d \lambda)
\end{aligned}
$$

as $|\mathcal{Z}| \longrightarrow 0$. Combining both relations completes the proof.

In what follows we assume that $\mathcal{H}=\mathcal{H}_{1}$.

Proposition 3.3 Let $X:[a, b) \longrightarrow \mathcal{B}(\mathcal{H})$ be an $F$-admissible function, and assume, in addition, that there exist real numbers $c_{1}, c_{2}$, such that $X(\lambda)$ is selfadjoint and $c_{1} \leq X(\lambda) \leq c_{2}, \lambda \in \Delta$. Let $\varphi \in C\left[c_{1}, c_{2}\right]$. Then the following holds:

(i) The operator $\widehat{X}:=\int_{\Delta} X(\lambda) F(d \lambda)$ is self-adjoint and satisfies $c_{1} \leq \widehat{X} \leq c_{2}$;

(ii) The following estimate holds $\|\varphi(\widehat{X})\| \leq\|\varphi\|_{\infty}$

(iii) The operator-valued function $\varphi(X(\cdot))$ is $F$-admissible and

$$
\int_{\Delta} \varphi(X(\lambda)) F(d \lambda)=\varphi(\widehat{X})
$$

Proof. (i) Let $\mathcal{Z}$ be any partition as above. Then for any $h \in \mathcal{H}$ one gets

$$
\left\langle\Sigma_{\mathcal{Z}} X h, h\right\rangle=\sum_{m=1}^{n}\left\langle F\left(\Delta_{m}\right) X\left(x_{m}\right) F\left(\Delta_{m}\right) h, h\right\rangle \geq \sum_{m=1}^{n} c_{1}\left\|F\left(\Delta_{m}\right) h\right\|^{2} .
$$

Thus $\left\langle\Sigma_{\mathcal{Z}} h, h\right\rangle \in \mathbb{R}$ and $\left\langle\Sigma_{\mathcal{Z}} h, h\right\rangle \geq c_{1}\|h\|^{2}$. In the same way one shows that $\left\langle\Sigma_{\mathcal{Z}} h, h\right\rangle \leq c_{2}\|h\|^{2}$. By passing to the limit, as $|\mathcal{Z}| \longrightarrow 0$, we get that $\langle\hat{X} h, h\rangle \in\left[c_{1}\|h\|^{2}, c_{2}\|h\|^{2}\right]$ for every $h \in \mathcal{H}$, and (i) is proved.

(ii) By the functional calculus, both inequalities $\|\varphi(\widehat{X})\| \leq\|\varphi\|_{\infty}$ and $\|\varphi(X(\lambda))\| \leq\|\varphi\|_{\infty}$ hold for every $\lambda \in \Delta$ and each continuous function $\varphi \in C\left[c_{1}, c_{2}\right]$. 
(iii) First we prove, by induction, the assertion (iii) in the special case, when $\varphi(\lambda)=\lambda^{n}$. By the assumption, the assertion is true for $n=1$. Suppose that it is true for $n=k$. Let us prove it for $n=k+1$. One has

$$
\begin{aligned}
& X^{k+1}(\lambda) F(\delta)=X(\lambda) F(\delta) X^{k}(\lambda) F(\delta) \\
& \quad=F(\delta) X(\lambda) F(\delta) \cdot F(\delta) X^{k}(\lambda) F(\delta)=F(\delta) X^{k+1}(\lambda) F(\delta),
\end{aligned}
$$

$\lambda \in \Delta, \delta \in \mathcal{B}(\Delta)$. Therefore Proposition 3.2 ensures that the integral $\int_{\Delta} X^{k+1}(\lambda) F(d \lambda)$ exists and

$$
\int_{\Delta} X^{k+1}(\lambda) F(d \lambda)=\widehat{X}^{k+1}
$$

By linearity, these equalities are easily extended for polynomials in $\lambda$.

Let $\varphi$ be a continuous function, $\varphi \in C\left[c_{1}, c_{2}\right]$. By the Weierstrass Theorem, there exists a sequence $\left\{p_{k}\right\}_{1}^{\infty}$ of polynomials approaching $\varphi$ in $C\left[c_{1}, c_{2}\right]$. In accordance with the functional calculus for self-adjoint operators,

$$
\left\|\varphi(\widehat{X})-p_{k}(\widehat{X})\right\| \leq\left\|\varphi-p_{k}\right\|_{\infty} \longrightarrow 0 \quad \text { as } \quad k \rightarrow \infty
$$

and

$$
\left\|\varphi(X(\lambda))-p_{k}(X(\lambda))\right\| \leq\left\|\varphi-p_{k}\right\|_{\infty} \longrightarrow 0 \quad \text { as } \quad k \rightarrow \infty, \quad \lambda \in \Delta .
$$

Combining this relation with equalities (3.4) for polynomials we obtain that for every $\lambda \in \Delta$ and any Borel subset $\delta \subset \Delta$ the following holds

$$
\begin{aligned}
\varphi(X(\lambda)) F(\delta) & =\lim _{k \longrightarrow \infty} p_{k}(X(\lambda)) F(\delta) \\
& =\lim _{k \longrightarrow \infty} F(\delta) p_{k}(X(\lambda)) F(\delta)=F(\delta) \varphi(X(\lambda)) F(\delta), \quad \lambda \in \Delta .
\end{aligned}
$$

This relation means that the function $\varphi(X(\cdot))$ satisfies commutation relation (3.2). To prove its $F$-admissibility it remains to prove the existence of the integral $\int_{\Delta} \varphi(X(\lambda)) F(d \lambda)$. We prove it together with relation (3.3). To this end for each partition $\mathcal{Z}$ of $[a, b]$ we prove the following estimate

$$
\left\|\Sigma_{\mathcal{Z}}\left(\varphi(X)-p_{k}(X)\right)\right\| \leq\left\|\varphi-p_{k}\right\|_{\infty} .
$$

Since the measure $F$ is orthogonal, one gets

$$
\begin{aligned}
\left\|\Sigma_{\mathcal{Z}} \varphi(X) f-\Sigma_{\mathcal{Z}} p_{k}(X) f\right\|^{2}=\left\|\sum_{m=1}^{n}\left(\varphi-p_{k}\right)(X)\left(x_{m}\right) F\left(\Delta_{m}\right) f\right\|^{2} \\
\quad=\sum_{m=1}^{n}\left\|\left(\varphi-p_{k}\right)(X)\left(x_{m}\right) F\left(\Delta_{m}\right) f\right\|^{2} \\
\leq \sum_{m=1}^{n}\left\|\varphi-p_{k}\right\|_{\infty}^{2}\left\|F\left(\Delta_{m}\right) f\right\|^{2}=\left\|\varphi-p_{k}\right\|_{\infty}^{2}\|f\|^{2} .
\end{aligned}
$$


Let $\left\{\mathcal{Z}_{j}\right\}$ be a sequence of partitions satisfying $\left|\mathcal{Z}_{j}\right| \longrightarrow 0$ and let $\varepsilon>0$. Choose $k$ such that $\left\|\varphi-p_{k}\right\|_{\infty}<\varepsilon$ and $j_{0}$ such that

$$
\left\|\Sigma_{\mathcal{Z}_{j}} p_{k}(X)-\Sigma_{\mathcal{Z}_{j^{\prime}}} p_{k}(X)\right\|<\varepsilon, \quad j, j^{\prime} \geq j_{0},
$$

and hence $\left\|\Sigma_{\mathcal{Z}_{j}} \varphi(X)-\Sigma_{\mathcal{Z}_{j^{\prime}}} \varphi(X)\right\|<3 \varepsilon$ for all $j, j^{\prime} \geq j_{0}$. Thus the limit $\lim _{j \longrightarrow \infty} \Sigma_{\mathcal{Z}_{j}} \varphi(X)$ exists, and

$$
\begin{aligned}
& \left\|\lim _{j \longrightarrow \infty} \Sigma_{\mathcal{Z}_{j}} \varphi(X)-\varphi(\widehat{X})\right\| \\
& \quad \leq\left\|\lim _{j \rightarrow \infty} \Sigma_{\mathcal{Z}_{j}}\left(\varphi(X)-p_{k}(X)\right)\right\|+\left\|p_{k}(\widehat{X})-\varphi(\widehat{X})\right\|<2 \varepsilon .
\end{aligned}
$$

Since $\varepsilon>0$ is arbitrary, this inequality ensures the existence of the integral $\int_{\Delta} \varphi(X(\lambda)) F(d \lambda)$, thus proves $F$-admissibility of $\varphi(X(\cdot))$. Moreover, estimate (3.5) proves equality (3.3).

Denote by $d m$ the Lebesgue measure on $\mathbb{R}$.

Corollary 3.4 Assume that $\Omega(\cdot)=\Omega(\cdot)^{*}$ is a self-adjoint $\mathcal{B}(\mathcal{H})$-valued Lipschitz function in $\Delta=[a, b)$ and $c_{1} \leq \Omega(\cdot) \leq c_{2}$. Assume also that $F(\cdot)$ is a spectral measure in $\mathcal{H}$ with compact support, $\operatorname{supp}(F) \subseteq \Delta:=[a, b)$, and $\varphi \in C\left[c_{1}, c_{2}\right]$. If in addition, commutation relation (3.2) holds, then the operator-valued function $\varphi(\Omega(\cdot))$ is $F$-admissible and

$$
\int_{\Delta} \varphi(\Omega(\lambda)) F(d \lambda)=\varphi(\widehat{X}) .
$$

Proof. It is shown in [3, Lemma 7.2] that the integral (3.1) exists whenever $\Omega(\cdot)$ is Lipschitz function. By Proposition 3.3(iii) $\varphi(\Omega(\cdot))$ is $F$-admissible and equality (3.6) holds.

Corollary 3.5 Let $\Omega(\cdot)=\Omega(\cdot)^{*}$ be differentiable with respect to the operator norm $m$-almost everywhere in $\Delta=[a, b), c_{1} \leq \Omega(\cdot) \leq c_{2}$, and let $\Omega(\cdot)$ be expressed by means of its derivative $\Omega^{\prime}(\cdot)$ via the Bochner integral on $[a, b)$, i.e.

$$
\Omega(\lambda)=\Omega(a)+\int_{a}^{\lambda} \Omega^{\prime}(x) d x, \quad \lambda \in[a, b) .
$$

Assume also that $F(\cdot)$ is a spectral measure in $\mathcal{H}$ with compact support, $\operatorname{supp}(F) \subseteq \Delta:=[a, b)$, and $\varphi \in C\left[c_{1}, c_{2}\right]$. Assume also that commutation relation (3.2) holds. Then the operator-valued function $\varphi(\Omega(\cdot))$ is $F$-admissible and

$$
\int_{\Delta} \varphi(\Omega(\lambda)) F(d \lambda)=\varphi(\widehat{X}) .
$$

Proof. It is known (see [7, Proposition 5.1.4]) that the integral (3.1) exists whenever $\Omega(\cdot)$ admits representation (3.7). By Proposition 3.3(iii) $\varphi(X(\cdot))$ is $F$-admissible and equality (3.8) holds. 
Remark 3.6 Absolute continuity of $\Omega(\cdot)$ (and even its Lipschitz property) does not ensure representation (3.7) (see [60, Chapter 5]). Thus, the conditions in both corollaries are different.

If $F(\cdot)$ is a spectral measure on $\mathbb{R}$ with non-compact support, then we define improper operator spectral integrals by

$$
\begin{gathered}
\int_{\mathbb{R}} \Omega(\lambda) F(d \lambda):=\mathrm{s}-\lim _{\substack{b \rightarrow+\infty \\
a \rightarrow-\infty}} \int_{\Delta} \Omega(\lambda) F(d \lambda), \\
\int_{\mathbb{R}} F(d \lambda) \Omega(\lambda):=\mathrm{s}-\lim _{\substack{b \rightarrow+\infty \\
a \rightarrow-\infty}} \int_{\Delta} F(d \lambda) \Omega(\lambda) .
\end{gathered}
$$

Obviously, the improper operator spectral integral $\int_{\mathbb{R}} \Omega(\lambda) F(d \lambda)$ exists if and only if the following conditions

$$
\text { s- } \lim _{b \rightarrow \infty} \int_{b}^{b+\varepsilon} \Omega(\lambda) F(d \lambda)=0 \quad \text { and } \quad \text { s- } \lim _{a \rightarrow-\infty} \int_{a-\varepsilon}^{a} \Omega(\lambda) F(d \lambda)=0,
$$

are satisfied for any $\varepsilon>0$. Similar results hold true for $\int_{\mathbb{R}} F(d \lambda) \Omega(\lambda)$.

Proposition 3.7 Let $\Omega: \mathbb{R} \longrightarrow \mathcal{B}(\mathcal{H})$. Assume that $\Omega \uparrow \Delta$ is $F$-admissible for every compact interval $\Delta$ and

$$
\|\Omega(\lambda)\| \leq C_{0}(1+|\lambda|)^{\alpha}, \quad \lambda \in \mathbb{R}
$$

for some constants $\alpha \geq 0, C_{0}>0$. Then the improper spectral integral $\int_{\mathbb{R}} \Omega(\lambda) F(d \lambda) f$ exists for any $f \in \mathcal{H}$ satisfying

$$
\int_{\mathbb{R}}|\lambda|^{2 \alpha} d\|F(\lambda) f\|^{2}<\infty
$$

Proof. Let $b, c>0$. Let $n \in \mathbb{N}$. Put $x_{m}:=b+\frac{m-1}{n} c, \Delta_{m}:=\left[x_{m}, x_{m}+\frac{c}{n}\right)$, $\mathcal{Z}:=\bigcup_{m=1}^{n} \Delta_{m}$. Then

$$
\begin{aligned}
\left\|\Sigma_{\mathcal{Z}} \Omega f\right\|^{2} & =\Sigma_{m=1}^{n}\left\|\Omega\left(x_{m}\right) F\left(\Delta_{m}\right) f\right\|^{2} \\
& \leq C_{0}^{2} \Sigma_{m=1}^{n}\left(1+x_{m}\right)^{2 \alpha}\left\|F\left(\Delta_{m}\right) f\right\|^{2} \\
& \leq C_{0}^{2} \int_{[b, b+c)}(1+\lambda)^{2 \alpha} d\|F(\lambda) f\|^{2} .
\end{aligned}
$$

Passing to the limit, as $n$ tends to infinity, we get that

$$
\left\|\int_{[b, b+c)} \Omega(\lambda) F(d \lambda) f\right\|^{2} \leq C_{0}^{2} \int_{[b, b+c)}(1+\lambda)^{2 \alpha} d\|F(\lambda) f\|^{2} .
$$

The integral on the right hand side tends to zero, as $b$ tends to infinity, provided (3.9) holds. The case $a \longrightarrow-\infty$ is treated similarly. 


\section{Boundary triplets for tensor products}

\subsection{Bounded case}

Let $A$ be a densely defined symmetric operator with equal deficiency indices acting in the separable Hilbert space $\mathfrak{H}$ and let $T$ be a bounded self-adjoint operator acting on the separable Hilbert space $\mathfrak{T}$. Let us consider the closed symmetric operator $S:=A \otimes I_{\mathfrak{T}}+I_{\mathfrak{H}} \otimes T$ in $\mathfrak{H}_{S}:=\mathfrak{H} \otimes \mathfrak{T}$. We recall that the operator $S$ is defined as the closure of $S_{\odot}:=A \odot I_{\mathfrak{T}}+I_{\mathfrak{H}} \odot T$,

$$
\operatorname{dom}\left(A \odot I_{\mathfrak{T}}+I_{\mathfrak{H}} \odot T\right):=\left\{f=\sum_{k=1}^{n} g_{k} \otimes h_{k}: g_{k} \in \operatorname{dom}(A), h_{k} \in \mathfrak{T}\right\}
$$

and

$$
S_{\odot} f:=\sum_{k=1}^{n}\left(A g_{k} \otimes h_{k}+g_{k} \otimes T h_{k}\right), \quad f \in \operatorname{dom}\left(A \odot I_{\mathfrak{T}}+I_{\mathfrak{H}} \odot T\right) .
$$

Obviously, the operator $S_{\odot}$ is densely defined and symmetric.

Let $\Pi_{A}=\left\{\mathcal{H}^{A}, \Gamma_{0}^{A}, \Gamma_{1}^{A}\right\}$ be a boundary triplet for $A^{*}$ with $\gamma$-field $\gamma^{A}(\cdot)$ and Weyl function $M^{A}(\cdot)$. Let $J_{A^{*}}$ be the embedding operator $J_{A^{*}}: \mathfrak{H}_{+}\left(A^{*}\right) \longrightarrow$ $\operatorname{dom}\left(A^{*}\right)$. Obviously, $\operatorname{ran}\left(J_{A^{*}}\right)=\operatorname{dom}\left(A^{*}\right)$ and $\operatorname{ker}\left(J_{A^{*}}\right)=\{0\}$ as well as $\Gamma_{j}^{A}=\widehat{\Gamma}_{j}^{A} J_{A^{*}}^{-1}, j=0,1$. Notice that $\mathfrak{H}_{+}\left(\left(A \otimes I_{\mathfrak{T}}\right)^{*}\right)=\mathfrak{H}_{+}\left(A^{*} \otimes I_{\mathfrak{T}}\right)=\mathfrak{H}_{+}\left(A^{*}\right) \otimes \mathfrak{T}$ and $J_{\left(A \otimes I_{\mathfrak{T}}\right)^{*}}=J_{A^{*} \otimes I_{\mathfrak{T}}}$. Moreover, one has

$$
\operatorname{ran}\left(J_{\left(A \otimes I_{\mathfrak{T}}\right)^{*}}\right)=\operatorname{dom}\left(\left(A \otimes I_{\mathfrak{T}}\right)^{*}\right)=\operatorname{dom}\left(A^{*} \otimes I_{\mathfrak{T}}\right) .
$$

We set

$$
\left(\Gamma_{j}^{A} \widehat{\otimes} I_{\mathfrak{T}}\right) f:=\left(\widehat{\Gamma}_{j}^{A} \otimes I_{\mathfrak{T}}\right) J_{\left(A \otimes I_{\mathfrak{T}}\right)^{*}}^{-1}, \quad j \in\{0,1\}, \quad f \in \operatorname{dom}\left(A^{*} \otimes I_{\mathfrak{T}}\right) .
$$

It turns out that $\Pi_{A} \widehat{\otimes} I_{\mathfrak{T}}:=\left\{\mathcal{H}^{A} \otimes \mathfrak{T}, \Gamma_{0}^{A} \widehat{\otimes} I_{\mathfrak{T}}, \Gamma_{1}^{A} \widehat{\otimes} I_{\mathfrak{T}}\right\}$ is a boundary triplet for $\left(A \otimes I_{\mathfrak{T}}\right)^{*}=A^{*} \otimes I_{\mathfrak{T}}$.

Theorem 4.1 Let $\Pi_{A}=\left\{\mathcal{H}^{A}, \Gamma_{0}^{A}, \Gamma_{1}^{A}\right\}$ be a boundary triplet for $A^{*}$ with $\gamma$ field $\gamma^{A}(\cdot)$ and Weyl function $M^{A}(\cdot)$. Let also $T=T^{*} \in \mathcal{B}(\mathfrak{T})$, and let $\Delta$ be the smallest closed interval containing the spectrum $\sigma(T)$. Finally, let $\widehat{E}_{T}(\delta):=$ $I_{\mathcal{H}^{A}} \otimes E_{T}(\delta), \delta \in \mathcal{B}(\mathbb{R})$, where $E_{T}(\cdot)$ is the spectral measure of $T$. Then:

(i) $\Pi_{S}=\left\{\mathcal{H}^{S}, \Gamma_{0}^{S}, \Gamma_{1}^{S}\right\}:=\Pi_{A} \widehat{\otimes} I_{\mathfrak{T}}$ is a boundary triplet for $S^{*}$ such that $S_{0}:=S^{*} \uparrow \operatorname{ker}\left(\Gamma_{0}^{S}\right)=A_{0} \otimes I_{\mathfrak{T}}+I_{\mathfrak{H}} \otimes T$.

(ii) The $\gamma$-field $\gamma^{S}(\cdot)$ and the Weyl function $M^{S}(\cdot)$ of $\Pi_{S}$ admit the following representations

$$
\gamma^{S}(z)=\int_{\Delta}\left(\gamma^{A}(z-\lambda) \otimes I_{\mathfrak{T}}\right) \widehat{E}_{T}(d \lambda), \quad z \in \mathbb{C}_{ \pm}
$$


and

$$
\begin{aligned}
M^{S}(z) & =\int_{\Delta} \widehat{E}_{T}(d \lambda)\left(M^{A}(z-\lambda) \otimes I_{\mathfrak{T}}\right) \\
& =\int_{\Delta}\left(M^{A}(z-\lambda) \otimes I_{\mathfrak{T}}\right) \widehat{E}_{T}(d \lambda), \quad z \in \mathbb{C}_{ \pm} .
\end{aligned}
$$

In particular,

$$
\operatorname{ran}\left(\int_{\Delta}\left(\gamma^{A}(z-\lambda) \otimes I_{\mathfrak{T}}\right) \widehat{E}_{T}(d \lambda)\right)=\mathfrak{N}_{z}\left(S^{*}\right)=\operatorname{ker}\left(S^{*}-z\right) .
$$

(iii) If the Weyl function $M^{A}(\cdot)$ is of scalar type, $M^{A}(\cdot)=m^{A}(\cdot) I_{\mathcal{H}^{A}}$, then

$$
M^{S}(z)=I_{\mathcal{H}^{A}} \otimes m^{A}(z-T), \quad z \in \mathbb{C}_{ \pm} .
$$

In particular, the latter holds whenever $n_{ \pm}(A)=1$.

Note that the integrals (4.2) and (4.1) exist due to Corollary 3.4 since both the Weyl function $M^{S}(z-\cdot)$ and $\gamma$-field $\gamma^{S}(z-\cdot)$ are holomorphic in $\lambda$, hence Lipschitz functions.

Proof. (i) The proof is straightforward.

(ii) In accordance with [3, Lemma 7.2] both integrals (4.1) and (4.2) exist since $\gamma^{A}(\cdot)$ and $M^{A}(\cdot)$ are Lipschitz. Let $\pi=\left\{a=\lambda_{0}<\lambda_{1}<\lambda_{2}<\ldots<\lambda_{n}=\right.$ $b\}$ be a partition of $\Delta=[a, b], \Delta_{k}:=\left[\lambda_{k-1}, \lambda_{k}\right)$, and let

$$
\begin{aligned}
T_{k} & :=\lambda_{k} E\left(\Delta_{k}\right), \quad T_{\pi}:=\bigoplus_{k=1}^{n} T_{k}=\sum_{k=1}^{n} \lambda_{k} E_{T}\left(\Delta_{k}\right), \\
S_{\pi} & :=A \otimes I_{\mathfrak{T}}+I_{\mathfrak{H}} \otimes T_{\pi},
\end{aligned}
$$

and $\mathfrak{T}_{k}:=\operatorname{ran} E\left(\Delta_{k}\right) . T_{k}$ is regarded as an operator in $\mathfrak{T}_{k}$. It is easily seen that $\mathfrak{T}=\bigoplus_{1}^{n} \mathfrak{T}_{k}$ and

$$
S_{\pi}=\bigoplus_{k=1}^{n} S_{k}, \quad S_{k}:=A \otimes I_{\mathfrak{T}_{k}}+I_{\mathfrak{H}} \otimes T_{k} \in \mathcal{C}\left(\mathfrak{H} \otimes \mathfrak{T}_{k}\right)
$$

Clearly, $S_{k}^{*}:=A^{*} \otimes I_{\mathfrak{T}_{k}}+I_{\mathfrak{H}} \otimes T_{k}$. Moreover for every $k$ such that $\mathfrak{T}_{k} \neq\{0\}$ we have $\sigma\left(T_{k}\right)=\left\{\lambda_{k}\right\}$ and hence $\sigma\left(S_{k}^{*}\right)=\sigma\left(A^{*} \otimes I_{\mathfrak{T}_{k}}\right)+\lambda_{k}$ and $\mathfrak{N}_{z}\left(S_{k}\right)=$ $\mathfrak{N}_{z-\lambda_{k}}(A) \otimes \mathfrak{T}_{k}$. Clearly,

$$
\begin{aligned}
S_{\pi}^{*} & =A^{*} \otimes\left(\bigoplus_{k=1}^{n} E_{T}\left(\Delta_{k}\right)\right)+I_{\mathfrak{H}} \otimes\left(\bigoplus_{k=1}^{n} \lambda_{k} E_{T}\left(\Delta_{k}\right)\right) \\
& =\bigoplus_{k=1}^{n}\left(A^{*}+\lambda_{k} I_{\mathfrak{H}}\right) \otimes E_{T}\left(\Delta_{k}\right) .
\end{aligned}
$$

Hence $\mathfrak{N}_{z}\left(S_{\pi}\right)=\operatorname{ker}\left(S_{\pi}^{*}-z I_{\mathfrak{H}}\right)=\operatorname{ran}\left(\sum_{k=1}^{n} \gamma^{A}\left(z-\lambda_{k}\right) \otimes E_{T}\left(\Delta_{k}\right)\right)$ 
Noting that $\Gamma_{0}^{S_{\pi}}=\Gamma_{0}^{S}=\Gamma_{0}^{A} \otimes I_{\mathfrak{T}}$ and using definition (2.3) one gets

$$
\begin{aligned}
\Gamma_{0}^{S_{\pi}} & \left(\sum_{k=1}^{n} \gamma^{A}(z-\lambda) \otimes E_{T}\left(\Delta_{k}\right)\right)=\sum_{k=1}^{n} \Gamma_{0}^{A} \gamma^{A}\left(z-\lambda_{k}\right) \otimes E_{T}\left(\Delta_{k}\right) \\
= & \sum_{k=1}^{n} I_{\mathcal{H}} \otimes E_{T}\left(\Delta_{k}\right)=I_{\mathcal{H}} \otimes I_{\mathfrak{T}}=I_{\mathcal{H} \otimes \mathfrak{T}}
\end{aligned}
$$

Combining this relation with definition (2.3) of the $\gamma$-field one derives

$$
\gamma^{S_{\pi}}(z)=\left(\Gamma_{0}^{S_{\pi}}\left\lceil\mathfrak{N}_{z}\left(S_{\pi}\right)\right)^{-1}=\sum_{k=1}^{n} \gamma^{A}\left(z-\lambda_{k}\right) \otimes E_{T}\left(\Delta_{k}\right) .\right.
$$

Applying operator $\Gamma_{1}$ to this equality and using Definition 2.4 we arrive at the Weyl function $M^{S_{\pi}}(\cdot)$ corresponding to the triplet $\Pi^{S_{\pi}}$ of $S_{\pi}^{*}$,

$$
\begin{aligned}
M^{S_{\pi}}(z) & =\Gamma_{1}^{S_{\pi}} \gamma^{S_{\pi}}(z)=\sum_{k=1}^{n} \Gamma_{1}^{A} \gamma^{A}\left(z-\lambda_{k}\right) \otimes E_{T}\left(\Delta_{k}\right) \\
& =\sum_{k=1}^{n} M^{A}\left(z-\lambda_{k}\right) \otimes E_{T}\left(\Delta_{k}\right) .
\end{aligned}
$$

Since the integrals (5.1) and (5.2) exist, the following uniform convergence holds

$$
\gamma^{S_{\pi}}(z) \rightarrow \int_{\Delta}\left(\gamma^{A}(z-\lambda) \otimes I_{\mathfrak{T}}\right) \widehat{E}_{T}(d \lambda)=: \widetilde{\gamma}^{S}(z) \quad \text { as } \quad|\pi| \rightarrow 0,
$$

and

$$
M^{S_{\pi}}(z) \rightarrow \int_{\Delta}\left(M^{A}(z-\lambda) \otimes I_{\mathfrak{T}}\right) \widehat{E}_{T}(d \lambda)=: \widetilde{M}^{S}(z) \quad \text { as } \quad|\pi| \rightarrow 0,
$$

where as usual $|\pi|=\max _{k=1,2, \ldots, n}\left|\Delta_{k}\right|$.

Next we show that $\widetilde{\gamma}^{S}(z)=\gamma^{S}(z)$ and $\widetilde{M}^{S}(z)=M^{S}(z)$ for $z \in \mathbb{C}_{ \pm}$. One gets

$$
\begin{aligned}
& \left(\left(A^{*}-z\right) \otimes I_{\mathfrak{T}}\right) \gamma^{S_{\pi}}(z) g=\sum_{k=1}^{n}\left(A^{*}-z\right) \gamma^{A}\left(z-\lambda_{k}\right) \otimes E_{T}\left(\Delta_{k}\right) g \\
& \quad=-\sum_{k=1}^{n} \lambda_{k} \gamma^{A}\left(z-\lambda_{k}\right) \otimes E_{T}\left(\Delta_{k}\right) g \rightarrow-\int_{\Delta}\left(\lambda \gamma^{A}(z-\lambda) \otimes I_{\mathfrak{T}}\right) \widehat{E}_{T}(d \lambda) g
\end{aligned}
$$

as $|\pi| \rightarrow 0$. Since $A^{*}$ is closed, one gets by combining this relation with (4.5) that $\int_{\Delta}\left(\lambda \gamma^{A}(z-\lambda) \otimes I_{\mathfrak{T}}\right) \widehat{E}_{T}(d \lambda) g \in \operatorname{dom}\left(A^{*} \otimes I_{\mathfrak{T}}\right)$ for each $g \in \mathcal{H} \otimes \mathfrak{T}$ and

$$
\begin{aligned}
\left(\left(A^{*}-z\right) \otimes I_{\mathfrak{T}}\right) & \int_{\Delta}\left(\gamma^{A}(z-\lambda) \otimes I_{\mathfrak{T}}\right) \widehat{E}_{T}(d \lambda) \\
& =-\int_{\Delta}\left(\lambda \gamma^{A}(z-\lambda) \otimes I_{\mathfrak{T}}\right) \widehat{E}_{T}(d \lambda) .
\end{aligned}
$$


In turn, using this relation and applying Proposition 3.2 we derive

$$
\begin{aligned}
& \left(S^{*}-z\right) \int_{\Delta}\left(\gamma^{A}(z-\lambda) \otimes I_{\mathfrak{T}}\right) \widehat{E}_{T}(d \lambda) \\
& =\left(\left(A^{*}-z\right) \otimes I\right) \int_{\Delta}\left(\gamma^{A}(z-\lambda) \otimes I_{\mathfrak{T}}\right) \widehat{E}_{T}(d \lambda) \\
& \quad+\int_{\Delta} \lambda \widehat{E}_{T}(d \lambda) \cdot \int_{\Delta}\left(\gamma^{A}(z-\lambda) \otimes I_{\mathfrak{T}}\right) \widehat{E}_{T}(d \lambda) \\
& =-\int_{\Delta}\left(\lambda \gamma^{A}(z-\lambda) \otimes I_{\mathfrak{T}}\right) \widehat{E}_{T}(d \lambda)+\int_{\Delta}\left(\lambda \gamma^{A}(z-\lambda) \otimes I_{\mathfrak{T}}\right) \widehat{E}_{T}(d \lambda)=0 .
\end{aligned}
$$

It follows that $\operatorname{ran}\left(\int_{\Delta}\left(\gamma^{A}(z-\lambda) \otimes I_{\mathfrak{T}}\right) \widehat{E}_{T}(d \lambda)\right) \subset \mathfrak{N}_{z}\left(S^{*}\right)=\operatorname{ker}\left(S^{*}-z\right)$.

Let us show that the convergence in (4.5) holds in $\mathfrak{H}_{+}(S)$, i.e. in the graph norm.

Choose a sequence $\left\{\pi_{n}\right\}_{1}^{\infty}$ of partitions of $[a, b]$ such that $\lim _{n \rightarrow \infty}\left|\pi_{n}\right|=0$. Since the convergence in (4.5) is uniform, there exists a constant $C(z)>0$ depending on $z$ and not depending on $n$ and such that $\left\|\gamma^{S_{\pi_{n}}}(z)\right\| \leq C(z)$ for all $n$. Besides, for any $\varepsilon>0$ there exists $N=N(\varepsilon) \in \mathbb{N}$ such that $\left\|T_{\pi_{n}}-T\right\| \leq \varepsilon$ for $n \geq N$. Taking these relations into account one gets

$$
\begin{aligned}
& \left\|\left(S^{*}-z\right) \gamma^{S_{\pi}}(z) g\right\|=\left\|\left(S^{*}-z\right) \gamma^{S_{\pi}}(z) g-\left(S_{\pi}^{*}-z\right) \gamma^{S_{\pi}}(z) g\right\| \\
& \quad=\left\|\left(I \otimes\left(T-T_{\pi}\right)\right) \gamma^{S_{\pi}}(z) g\right\| \leq \varepsilon\left\|\gamma^{S_{\pi}}(z)\right\| \cdot\|g\| \leq \varepsilon C(z)\|g\|
\end{aligned}
$$

for any $\pi \in\left\{\pi_{n}\right\}_{N}^{\infty}$, hence $\left\|\lim _{n \rightarrow \infty}\left(S^{*}-z\right) \gamma^{S_{\pi_{n}}}(z) g\right\|=0$ for any $g \in \mathcal{H} \otimes \mathfrak{T}$. In turn, combining this relation with (4.5) yields

$$
\lim _{n \rightarrow \infty}\left\|\gamma^{S_{\pi_{n}}}(z)-\int_{\Delta}\left(\gamma^{A}(z-\lambda) \otimes I_{\mathfrak{T}}\right) \widehat{E}_{T}(d \lambda)\right\|_{\mathfrak{H}_{+}(S)}=0 .
$$

It follows from (4.4) that $\Gamma_{0}^{S} \gamma^{S_{\pi}}(z)=\Gamma_{0}^{S_{\pi}} \gamma^{S_{\pi}}(z)=I_{\mathcal{H}} \otimes I_{\mathfrak{T}} \rightarrow I_{\mathcal{H}} \otimes I_{\mathfrak{T}}$ as $|\pi| \rightarrow 0$. Therefore relation (4.7) implies

$$
\Gamma_{0}^{S} \widetilde{\gamma}^{S}(z)=I_{\mathcal{H}} \otimes I_{\mathfrak{T}},
$$

i.e. $\widetilde{\gamma}^{S}(z)=\gamma^{S}(z)$. This proves (4.1). In turn, (4.1) implies (4.3).

Further, combining just established relation $\widetilde{\gamma}^{S}(\cdot)=\gamma^{S}(\cdot)$ with relation (4.7) and using the boundedness of the operator $\Gamma_{1}^{S} \in\left[\mathfrak{H}_{+}(S), \mathcal{H}\right]$ we obtain

$$
\begin{aligned}
\lim _{n \rightarrow \infty} M^{S_{\pi_{n}}}(z) & =\lim _{n \rightarrow \infty} \Gamma_{1}^{S_{\pi_{n}}} \gamma^{S_{\pi_{n}}}(z) \\
& =\lim _{n \rightarrow \infty} \Gamma_{1}^{S} \gamma^{S_{\pi_{n}}}(z)=\Gamma_{1}^{S} \gamma^{S}(z)=M^{S}(z), \quad z \in \mathbb{C}_{ \pm}
\end{aligned}
$$

where the convergence is uniform. In turn, combining this relation with (4.6) yields (4.2).

Remark 4.2 Another proof of Theorem4.1 can be found in [14, cf. Proposition 3.1 and 3.2 . 
Example 4.3 Let us illustrate the theorem above. To this end we consider the case that $A$ is a closed symmetric operator with deficiency indices $n_{ \pm}=2$. In particular, let $\Pi_{A}=\left\{\mathcal{H}^{A}, \Gamma_{0}^{A}, \Gamma_{1}^{A}\right\}$ where $\mathcal{H}^{A}=\left(\mathcal{H}_{1}^{A} \oplus \mathcal{H}_{2}^{A}\right)^{t}, \mathcal{H}_{j}^{A}=\mathbb{C}, j=1,2$. We use the representation

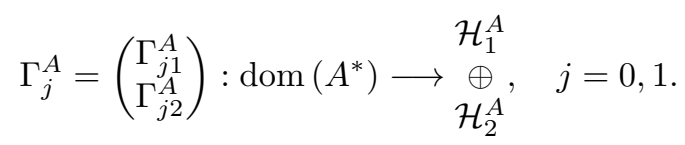

For the Gamma field $\gamma^{A}(\cdot)$ we use the representation $\gamma^{A}(z)=\left(\gamma_{1}^{A}(z), \gamma_{2}^{A}(z)\right)$, $\gamma_{j}^{A}(z): \mathcal{H}_{j}^{A} \longrightarrow \mathfrak{H}, j=1,2, z \in \mathbb{C}_{ \pm}$. The Weyl function $M^{A}(\cdot)$ admits the representation

$$
M^{A}(z)=\left(\begin{array}{ll}
m_{11}^{A}(z) & m_{12}^{A}(z) \\
m_{21}^{A}(z) & m_{22}^{A}(z)
\end{array}\right), \quad z \in \mathbb{C}_{ \pm}
$$

where $m_{i j}^{A}(\cdot)$ are holomorphic functions in $\mathbb{C}_{ \pm}$.

We consider the closed symmetric operator $S=A \otimes I_{\mathfrak{T}}+I_{\mathfrak{H}} \otimes T$, where $T$ is bounded and self-adjoint. Let $\Pi_{S}=\Pi_{A} \widehat{\otimes} I_{\mathfrak{T}}$, cf. Theorem 4.1](i). Obviously, the boundary value space $\mathcal{H}^{S}=\mathcal{H}^{A} \otimes \mathfrak{T}$ can be decomposed by $\mathcal{H}^{S}=\left(\mathcal{H}_{1}^{S} \oplus\right.$ $\left.\mathcal{H}_{2}^{S}\right)^{t}, \mathcal{H}_{j}^{S}:=\mathfrak{T}, j=1,2$. The boundary value maps $\Gamma_{0}^{S}=\Gamma_{0}^{A} \widehat{\otimes} I_{\mathfrak{T}}$ and $\Gamma_{1}^{S}=$ $\Gamma_{1}^{A} \widehat{\otimes} I_{\mathfrak{T}}$ will be represented by

$$
\Gamma_{0}^{S}=\left(\begin{array}{c}
\Gamma_{01}^{S} \\
\Gamma_{02}^{S}
\end{array}\right): \operatorname{dom}\left(S^{*}\right) \longrightarrow \underset{\mathcal{H}_{2}^{S}}{\stackrel{\mathcal{H}_{1}^{S}}{\oplus}} \text { and } \Gamma_{1}^{S}=\left(\begin{array}{c}
\Gamma_{11}^{S} \\
\Gamma_{12}^{S}
\end{array}\right): \operatorname{dom}\left(S^{*}\right) \longrightarrow \begin{gathered}
\mathcal{H}_{1}^{S} \\
\oplus \\
\mathcal{H}_{2}^{S}
\end{gathered}
$$

where $\Gamma_{0 j}^{S}:=\Gamma_{0 j}^{A} \widehat{\otimes} I_{\mathfrak{T}}$ and $\Gamma_{1 j}^{S}:=\Gamma_{0}^{A} \widehat{\otimes} I_{\mathfrak{T}}, j=0,1$. From (4.1) we get the representation $\gamma^{S}(z)=\left(\gamma_{1}^{S}(z), \gamma_{2}^{S}(z)\right), z \in \mathbb{C}_{ \pm}$, where $\gamma_{j}^{S}(z): \mathcal{H}_{j}^{S} \longrightarrow \mathfrak{H}$,

$$
\gamma_{j}^{S}(z):=\int_{\Delta} \gamma_{j}^{A}(z-\lambda) \widehat{E}_{T}(d \lambda), \quad j=1,2 .
$$

The Weyl function $M^{S}(\cdot)$ admits the representation

$$
M^{S}(z)=\left(\begin{array}{cc}
m_{11}^{A}(z-T) & m_{12}^{A}(z-T) \\
m_{21}^{A}(z-T) & m_{22}^{A}(z-T)
\end{array}\right): \underset{\mathcal{H}_{2}^{S}}{\stackrel{\mathcal{H}^{S}}{\oplus}} \rightarrow \underset{\mathcal{H}_{2}^{S}}{\oplus}, \quad z \in \mathbb{C}_{ \pm} .
$$

The representation of the Weyl function becomes very simple if $M^{A}(\cdot)$ is diagonal. In this case we have $M^{S}(z)=\operatorname{diag}\left(m_{11}^{A}(z-T), m_{22}^{A}(z-T)\right), z \in \mathbb{C}_{ \pm}$.

Let us compute the normalized boundary triplet $\widetilde{\Pi}_{S}$ associated with $\Pi_{S}$ in accordance with Lemma 2.6 .

Proposition 4.4 Let $\Pi_{A}=\left\{\mathcal{H}^{A}, \Gamma_{0}^{A}, \Gamma_{1}^{A}\right\}$ be a boundary triplet for $A^{*}$ with the $\gamma$-field $\gamma^{A}(\cdot)$ and Weyl function $M^{A}(\cdot)$. Let also $A_{0}:=A^{*} \uparrow \operatorname{ker}\left(\Gamma_{0}^{A}\right)$, $T=T^{*} \in \mathcal{B}(\mathfrak{T})$, and let $\Delta$ be the smallest closed interval containing the spectrum $\sigma(T)$, and let $\Pi_{S}=\Pi_{A} \widehat{\otimes} I_{\mathfrak{T}}$. Finally, let $\widehat{E}_{T}(\delta):=I_{\mathcal{H}^{A}} \otimes E_{T}(\delta), \delta \in \mathcal{B}(\mathbb{R})$, where $E_{T}(\cdot)$ is the spectral measure of $T$. Then: 
(i) The triplet $\widetilde{\Pi}_{S}=\left\{\widetilde{\mathcal{H}}^{S}, \widetilde{\Gamma}_{0}^{S}, \widetilde{\Gamma}_{1}^{S}\right\}$ with $\widetilde{\mathcal{H}}^{S}:=\mathcal{H}^{A} \otimes \mathfrak{T}$ and

$$
\begin{aligned}
\widetilde{\Gamma}_{0}^{S}:= & \left(\int_{\Delta} \widehat{E}_{T}(d \lambda) \sqrt{\operatorname{Im}\left(M^{A}(i-\lambda)\right)} \otimes I_{\mathfrak{T}}\right) \cdot\left(\Gamma_{0}^{A} \widehat{\otimes} I_{\mathfrak{T}}\right) \\
\widetilde{\Gamma}_{1}^{S}:= & \left(\int_{\Delta} \widehat{E}_{T}(d \lambda) \frac{1}{\sqrt{\operatorname{Im}\left(M^{A}(i-\lambda)\right)}} \otimes I_{\mathfrak{T}}\right) \cdot \\
& \cdot\left(\Gamma_{1}^{A} \widehat{\otimes} I_{\mathfrak{T}}-\left(\int_{\Delta} \widehat{E}_{T}(d \lambda) \operatorname{Re}\left(M^{A}(i-\lambda)\right) \otimes I_{\mathfrak{T}}\right) \cdot\left(\Gamma_{0}^{A} \widehat{\otimes} I_{\mathfrak{T}}\right)\right)
\end{aligned}
$$

forms a normalized boundary triplet for $S^{*}$ such that

$$
\widetilde{S}_{0}:=S^{*} \uparrow \operatorname{ker}\left(\widetilde{\Gamma}_{0}^{S}\right)=S_{0}=A_{0} \otimes I_{\mathfrak{T}}+I_{\mathfrak{H}} \otimes T .
$$

(ii) The $\gamma$-field $\widetilde{\gamma}^{S}(\cdot)$ and Weyl function $\widetilde{M}^{S}(\cdot)$ corresponding to the normalized boundary triplet $\widetilde{\Pi}_{S}$ admit the following representations

$$
\widetilde{\gamma}^{S}(z)=\int_{\Delta}\left(\gamma^{A}(z-\lambda) \frac{1}{\sqrt{\operatorname{Im}\left(M^{A}(i-\lambda)\right)}} \otimes I_{\mathfrak{T}}\right) \widehat{E}_{T}(d \lambda), \quad z \in \mathbb{C}_{ \pm},
$$

and

$$
\begin{aligned}
\widetilde{M}^{S}(z) & =\int_{\Delta}\left(L^{A}(z-\lambda, i-\lambda) \otimes I_{\mathfrak{T}}\right) \widehat{E}_{T}(d \lambda) \\
& =\int_{\Delta} \widehat{E}_{T}(d \lambda)\left(L^{A}(z-\lambda, i-\lambda) \otimes I_{\mathfrak{T}}\right), \quad z \in \mathbb{C}_{ \pm},
\end{aligned}
$$

where

$$
L^{A}(z, \zeta):=\frac{1}{\sqrt{\operatorname{Im}\left(M^{A}(\zeta)\right)}}\left(M^{A}(z)-\operatorname{Re}\left(M^{A}(\zeta)\right)\right) \frac{1}{\sqrt{\operatorname{Im}\left(M^{A}(\zeta)\right)}}
$$

for $z \in \mathbb{C}_{ \pm}, \zeta \in \mathbb{C}_{+}$.

(iii) If the Weyl function $M^{A}(\cdot)$ is of scalar type, $M^{A}(\cdot)=m^{A}(\cdot) I_{\mathcal{H}^{A}}, m^{A}(\cdot) \in$ $R[\mathbb{C}]$, then

$$
\widetilde{M}^{S}(z)=I_{\mathcal{H}^{A}} \otimes \frac{m^{A}(z-T)-\operatorname{Re}\left(m^{A}(i-T)\right)}{\operatorname{Im}\left(m^{A}(i-T)\right)}, \quad z \in \mathbb{C}_{ \pm} .
$$

In particular, the latter happen whenever $n_{ \pm}(A)=1$.

Proof. (i) By Theorem 4.1, $M^{S}(z)=\int_{\Delta} M^{A}(z-\lambda) \otimes I_{\mathcal{T}} \widehat{E}_{T}(d \lambda)$, and hence for each $z \in \mathbb{C}_{+}$

$$
\begin{aligned}
& \operatorname{Im}\left(M^{S}(z)\right)=\int_{\Delta}\left(\operatorname{Im}\left(M^{A}(z-\lambda)\right) \otimes I_{\mathcal{T}}\right) \widehat{E}_{T}(d \lambda) \quad \text { and } \\
& \operatorname{Re}\left(M^{S}(z)\right)=\int_{\Delta}\left(\operatorname{Re}\left(M^{A}(z-\lambda)\right) \otimes I_{\mathcal{T}}\right) \widehat{E}_{T}(d \lambda) .
\end{aligned}
$$


First we note that both integrals in (4.13) exist since the operator-valued functions $\operatorname{Im}\left(M^{A}(z-\cdot)\right)$ and $\operatorname{Re}\left(M^{A}(z-\cdot)\right.$ are Lipschitz (see [3]). Moreover, since the spectral measure $\widehat{E}_{T}=I_{\mathfrak{H}} \otimes E_{T}$ commutes with $M^{A}(z-\lambda) \otimes I_{\mathcal{T}}$, both functions $\operatorname{Im}\left(M^{A}(i-\cdot)\right) \otimes I_{\mathcal{T}}$ and $\operatorname{Re}\left(M^{A}(i-\cdot)\right) \otimes I_{\mathcal{T}}$ are $\widehat{E}_{T^{-}}$admissible. Noting that $M^{A}(\cdot)$ is holomorphic on $\mathbb{C}_{+}$and $0 \in \rho(\operatorname{Im} M(z))$ for $z \in \mathbb{C}_{+}$, one easily concludes that the operator-valued functions $\operatorname{Im}\left(M^{A}(i-\cdot)\right) \otimes I_{\mathcal{T}}, \operatorname{Re}\left(M^{A}(i-\cdot)\right) \otimes I_{\mathcal{T}}$, and $\left(\operatorname{Im}\left(M^{A}(i-\cdot)\right)^{-1} \otimes I_{\mathcal{T}}\right.$ are bounded on the compact set $\Delta$ and with some constants $c_{1}, c_{2}>0$ the following estimates hold

$$
\begin{aligned}
& 0<c_{1} \leq \operatorname{Im}\left(M^{A}(i-\lambda)\right) \otimes I_{\mathcal{T}} \leq c_{2} \quad \text { and } \\
& c_{2}^{-1} \leq\left(\operatorname{Im}\left(M^{A}(i-\lambda)\right)^{-1} \otimes I_{\mathcal{T}} \leq c_{1}^{-1}, \quad \lambda \in \Delta .\right.
\end{aligned}
$$

Since the function $\varphi(\cdot)=\sqrt{ }$ is continuous on $\mathbb{R}_{+}$, then in accordance with Proposition 3.3(iii) the compositions $\left(\operatorname{Im}\left(M^{A}(i-\lambda)\right)\right)^{1 / 2} \otimes I_{\mathcal{T}}$ and $\left(\operatorname{Im}\left(M^{A}(i-\lambda)\right)\right)^{-1 / 2} \otimes I_{\mathcal{T}}$ are $\widehat{E}_{T}$-admissible and

$$
\begin{aligned}
R & :=\sqrt{\operatorname{Im}\left(M^{S}(i)\right)}=\int_{\Delta}\left(\sqrt{\operatorname{Im}\left(M^{A}(i-\lambda)\right)} \otimes I_{\mathfrak{T}}\right) \widehat{E}_{T}(d \lambda), \\
R^{-1} & =\frac{1}{\sqrt{\operatorname{Im}\left(M^{S}(i)\right)}}=\int_{\Delta}\left(\frac{1}{\sqrt{\operatorname{Im}\left(M^{A}(i-\lambda)\right)}} \otimes I_{\mathfrak{T}}\right) \widehat{E}_{T}(d \lambda) .
\end{aligned}
$$

Combining the second formula in (4.14) with formula (4.2) and applying Proposition 3.2 one arrives at

$$
\begin{aligned}
R^{-1} Q & :=R^{-1} \operatorname{Re}\left(M^{S}(i)\right) \\
& =\int_{\Delta}\left(\frac{1}{\sqrt{\operatorname{Im}\left(M^{A}(i-\lambda)\right)}} \operatorname{Re}\left(M^{A}(i-\lambda)\right) \otimes I_{\mathfrak{T}}\right) \widehat{E}_{T}(d \lambda) .
\end{aligned}
$$

Now it follows from Lemma 2.6 (see formula (2.12) ) that a triplet $\widetilde{\Pi}_{S}=$ $\left\{\mathcal{H}_{S}, \widetilde{\Gamma}_{0}^{S}, \widetilde{\Gamma}_{1}^{S}\right\}$, where

$$
\widetilde{\Gamma}_{0}^{S}=\sqrt{\operatorname{Im}\left(M^{S}(i)\right)} \Gamma_{0}^{S} \quad \text { and } \quad \widetilde{\Gamma}_{1}^{S}=\frac{1}{\sqrt{\operatorname{Im}\left(M^{S}(i)\right)}}\left(\Gamma_{1}^{S}-\operatorname{Re}\left(M^{S}(i)\right) \Gamma_{0}^{S}\right),
$$

is a (normalized) boundary triplet for $S^{*}$. Combining these formulas with formulas (4.14) yields (4.8).

(ii) Combining (4.1) with the second identity in (4.14) and applying Proposition 3.2 we arrive at

$$
\begin{aligned}
& \widetilde{\gamma}^{S}(z)=\gamma^{S}(z) R^{-1} \\
& =\int_{\Delta}\left(\gamma^{A}(z-\lambda) \otimes I_{\mathfrak{T}}\right) \widehat{E}_{T}(d \lambda) \cdot \int_{\Delta}\left(\frac{1}{\sqrt{\operatorname{Im}\left(M^{A}(i-\mu)\right)}} \otimes I_{\mathfrak{T}}\right) \widehat{E}_{T}(d \mu) \\
& =\int_{\Delta}\left(\gamma^{A}(z-\lambda) \frac{1}{\sqrt{\operatorname{Im}\left(M^{A}(i-\lambda)\right)}} \otimes I_{\mathfrak{T}}\right) \widehat{E}_{T}(d \lambda), \quad z \in \mathbb{C}_{ \pm},
\end{aligned}
$$


which proves (4.9).

Similarly, combining formula (4.2) with the third formula in (4.14) and applying Proposition 3.2 implies

$$
\begin{aligned}
& \frac{1}{\sqrt{\operatorname{Im}\left(M^{S}(i)\right)}}\left(M^{S}(z)-\operatorname{Re}\left(M^{S}(i)\right)\right) \frac{1}{\sqrt{\operatorname{Im}\left(M^{S}(i)\right)}} \\
& =\int_{\Delta} \frac{1}{\sqrt{\operatorname{Im}\left(M^{A}(i-\lambda)\right)}} \times \\
& \quad \times\left(M^{A}(z-\lambda)-\operatorname{Re}\left(M^{A}(i-\lambda)\right) \frac{1}{\sqrt{\operatorname{Im}\left(M^{A}(i-\lambda)\right)}} \widehat{E}_{T}(d \lambda),\right.
\end{aligned}
$$

$z \in \mathbb{C}_{ \pm}$. This proves (4.10). Moreover, inserting in (4.10) $z=i$ one easily gets the equality $\widetilde{M}^{S}(i)=i\left(I_{\mathcal{H}^{A}} \otimes I_{\mathfrak{T}}\right)=i I_{\mathcal{H}^{S}}$ meaning that the triplet $\widetilde{\Pi}_{S}$ is normalized.

(iii) Representation (4.12) is immediate from (4.10).

\subsection{Unbounded case}

Let $A$ be a closed densely defined symmetric operator with equal deficiency indices in $\mathfrak{H}$ and let $T$ be an unbounded self-adjoint operator in $\mathfrak{T}$. First we introduce an operator $S^{\prime}:=A \odot I_{\mathfrak{T}}+I_{\mathfrak{H}} \odot T$ by setting (cf. [58, Chapter 7.5.2])

$$
\begin{aligned}
S^{\prime} f & :=A \odot I_{\mathfrak{T}} f+I_{\mathfrak{H}} \odot T f:=\sum_{k=1}^{l}\left(A g_{k} \otimes h_{k}\right)+\sum_{k=1}^{l}\left(g_{k} \otimes T h_{k}\right), \\
f & =\sum_{k=1}^{l} g_{k} \otimes h_{k} \in \operatorname{dom}\left(S^{\prime}\right), \\
\operatorname{dom}\left(S^{\prime}\right): & :=\left\{f=\sum_{k=1}^{l} g_{k} \otimes h_{k}: g_{k} \in \operatorname{dom}(A), \quad h_{k} \in \operatorname{dom}(T), l \in \mathbb{N}\right\} .
\end{aligned}
$$

Clearly, $S^{\prime}$ is a densely defined symmetric operator. Further, we define the operator $S:=A \otimes I_{\mathfrak{T}}+I_{\mathfrak{H}_{A}} \otimes T$ on $\mathfrak{K}:=\mathfrak{H} \otimes \mathfrak{T}$ as the closure of $S^{\prime}$, i.e.

$$
S:=\overline{S^{\prime}}:=\overline{A \odot I_{\mathfrak{T}}+I_{\mathfrak{H}} \odot T} .
$$

Denote by $\mathfrak{H}_{+}(A)$ the Hilbert space obtained by equipping the domain $\operatorname{dom}(A)$ with the graph norm. Let $J_{A}: \mathfrak{H}_{+}(A) \longrightarrow \mathfrak{H}$ be the embedding operator. Then $\operatorname{dom}\left(A \otimes I_{T}\right)=\left(J_{A} \otimes I_{\mathfrak{T}}\right)\left(\mathfrak{H}_{+}(A) \otimes \mathfrak{T}\right)$. By [58, Proposition 7.26], $\left(A \otimes I_{T}\right)^{*}=A^{*} \otimes I_{T}$ and

$$
\operatorname{dom}\left(A^{*} \otimes I_{T}\right)=\left(J_{A^{*}} \otimes I_{\mathfrak{T}}\right)\left(\mathfrak{H}_{+}\left(A^{*}\right) \otimes \mathfrak{T}\right) .
$$

The operator $I_{\mathfrak{H}} \otimes T=\overline{I_{\mathfrak{H}} \odot T}$ is unbounded and self-adjoint. Moreover. one has

$$
S=\overline{A \otimes I_{\mathfrak{T}}+I_{\mathfrak{H}} \otimes T} \quad \text { and } \quad \operatorname{dom}(S) \supseteq \mathcal{D}:=\operatorname{dom}\left(A \otimes I_{\mathfrak{T}}\right) \cap \operatorname{dom}\left(I_{\mathfrak{H}} \otimes T\right) .
$$


Clearly, $\mathcal{D}$ is a core for $S$, i.e. $S=\overline{S\lceil\mathcal{D}}$.

Further, setting $T_{n}:=E_{T}((n, n+1]) T$ and $\mathfrak{T}_{n}:=E_{T}((n, n+1]) \mathfrak{T}, n \in \mathbb{Z}$, one arrives at the orthogonal decomposition

$$
T=\bigoplus_{n \in \mathbb{Z}} T_{n}, \quad \mathfrak{T}:=\bigoplus_{n \in \mathbb{Z}} \mathfrak{T}_{n}
$$

where $T_{n}=T_{n}^{*} \in \mathcal{B}\left(\mathfrak{T}_{n}\right)$. Let $\mathfrak{K}_{n}:=\mathfrak{H} \otimes \mathfrak{T}_{n}, n \in \mathbb{Z}$. Clearly, $\mathfrak{K}:=\mathfrak{H} \otimes \mathfrak{T}=$ $\bigoplus_{n \in \mathbb{Z}} \mathfrak{K}_{n}$. We set $S_{n}:=A \otimes I_{\mathfrak{T}_{n}}+I_{\mathfrak{H}} \otimes T_{n}, n \in \mathbb{Z}$. For each $n \in \mathbb{Z}$ the operator $S_{n}$ is a well-defined closed symmetric operator in $\mathfrak{H}_{n}$.

Lemma 4.5 Let $A$ and $T$ be as above. Let $T=\bigoplus_{n \in \mathbb{Z}} T_{n}$ be an orthogonal decomposition of $T$ where $T_{n}=T_{n}^{*} \in \mathcal{B}\left(\mathfrak{T}_{n}\right)$. Then

$$
S=\bigoplus_{n \in \mathbb{Z}} S_{n}, \quad S_{n}:=A \otimes I_{\mathfrak{T}}+I_{\mathfrak{H}_{n}} \otimes T_{n} .
$$

In particular, if $T$ has a pure point spectrum, then $S=\bigoplus_{n \in \mathbb{Z}} S_{n}$ where $S_{n}=$ $A \otimes I_{\mathfrak{T}_{n}}+\lambda_{n} I_{\mathfrak{H}_{n}},\left\{\lambda_{n}\right\}_{n \in \mathbb{Z}}$ is the sequence of eigenvalues of $T$, and $\mathfrak{H}_{n}:=\mathfrak{H} \otimes \mathfrak{T}_{n}$ with $\mathfrak{T}_{n}=E_{T}\left(\left\{\lambda_{n}\right\}\right) \mathfrak{T}$.

Proof. The proof is obvious.

In general, for any self-adjoint extension $S_{0}$ of $S$ there is a boundary triplet $\Pi_{S}=\left\{\mathcal{H}^{S}, \widetilde{\Gamma}_{0}^{S}, \widetilde{\Gamma}_{1}^{S}\right\}$ such that $S_{0}=S^{*} \uparrow \operatorname{ker}\left(\Gamma_{0}^{S}\right)$. Moreover, in accordance with Lemma 2.6 it is always possible starting with a $\Pi_{S}$ to define a normalized boundary triplet $\widetilde{\Pi}_{S}$. In particular, we can find a boundary triplet $\Pi_{S}$ for $S^{*}$, $S=A \otimes I_{\mathfrak{T}}+I_{\mathfrak{H}} \otimes T$, such that $S_{0}:=A_{0} \otimes I_{\mathfrak{T}}+I_{\mathfrak{H}} \otimes T$. However, in applications we need a special boundary triplet feeling a tensor structure of the operators $S$ and $S^{*}$ and leading to simple forms of the corresponding Weyl function and $\gamma$-field.

Therefore in what follows we choose another strategy. Let $\Pi_{A}$ be a boundary triplet for $A^{*}$ with the corresponding $\gamma$-field $\gamma^{A}(\cdot)$ and Weyl function $M^{A}(\cdot)$. Starting with this boundary triplet for $A^{*}$ we construct a normalized boundary triplet $\Pi_{S}=\left\{\mathcal{H}^{S}, \Gamma_{0}^{S}, \Gamma_{1}^{S}\right\}$ for $S^{*}$ such that $S_{0}=S^{*}\left\lceil\operatorname{ker}\left(\Gamma_{0}^{S}\right)\right.$ and the corresponding $\gamma$-field $\gamma^{S}(\cdot)$ and Weyl function $M^{S}(\cdot)$ can be explicitly computed by means of $\gamma^{A}(\cdot)$ and $M^{A}(\cdot)$ (cf. the proof of Theorem 4.8).

Lemma 4.6 Let $A$ be a densely defined closed symmetric operator in $\mathfrak{H}$. Let also $\Pi_{A}=\left\{\mathcal{H}^{A}, \Gamma_{0}^{A}, \Gamma_{1}^{A}\right\}$ be a boundary triplet for $A^{*}$ and let $M^{A}(\cdot)$ and $\gamma(\cdot)$ be the corresponding Weyl function and $\gamma$-field, respectively. Further, let $T$ be a self-adjoint operator on $\mathfrak{T}$ with spectral measure $E_{T}(\cdot)$ and let $\widehat{E}_{T}(\cdot):=$ 
$I_{\mathcal{H}^{A}} \otimes E_{T}(\cdot)$. Then the following improper spectral integrals

$$
\begin{aligned}
G_{0} f & :=\int_{\mathbb{R}} \widehat{E}_{T}(d \lambda)\left(\sqrt{\operatorname{Im}\left(M^{A}(i-\lambda)\right)} \otimes I_{\mathfrak{T}}\right) f \\
& =\int_{\mathbb{R}}\left(\sqrt{\operatorname{Im}\left(M^{A}(i-\lambda)\right)} \otimes I_{\mathfrak{T}}\right) \widehat{E}_{T}(d \lambda) f \\
G_{1} f & :=\int_{\mathbb{R}} \widehat{E}_{T}(d \lambda)\left(\frac{1}{\sqrt{\operatorname{Im}\left(M^{A}(i-\lambda)\right)}} \otimes I_{\mathfrak{T}}\right) f \\
& =\int_{\mathbb{R}}\left(\frac{1}{\sqrt{\operatorname{Im}\left(M^{A}(i-\lambda)\right)} \otimes I_{\mathfrak{T}}}\right) \widehat{E}_{T}(d \lambda) f, \\
G_{2} f & :=\int_{\mathbb{R}} \widehat{E}_{T}(d \lambda)\left(\frac{1}{\sqrt{\operatorname{Im}\left(M^{A}(i-\lambda)\right)}} \operatorname{Re}\left(M^{A}(i-\lambda)\right) \otimes I_{\mathfrak{T}}\right) f \\
& =\int_{\mathbb{R}}\left(\frac{1}{\sqrt{\operatorname{Im}\left(M^{A}(i-\lambda)\right)}} \operatorname{Re}\left(M^{A}(i-\lambda)\right) \otimes I_{\mathfrak{T}}\right) \widehat{E}_{T}(d \lambda) f
\end{aligned}
$$

exist for each $f \in \operatorname{dom}\left(I_{\mathcal{H}^{A}} \otimes T\right)$. Moreover, the following improper spectral integrals

$$
G(z) f:=\int_{\mathbb{R}}\left(\gamma^{A}(z-\lambda) \frac{1}{\sqrt{\operatorname{Im}\left(M^{A}(i-\lambda)\right)}} \otimes I_{\mathfrak{T}}\right) \widehat{E}_{T}(d \lambda) f,
$$

and

$$
\begin{aligned}
M(z) f & :=\int_{\mathbb{R}}\left(L^{A}(z-\lambda, i-\lambda) \otimes I_{\mathfrak{T}}\right) \widehat{E}_{T}(d \lambda) f \\
& =\int_{\mathbb{R}} \widehat{E}_{T}(d \lambda)\left(L^{A}(z-\lambda, i-\lambda) \otimes I_{\mathfrak{T}}\right) f, \quad z \in \mathbb{C}_{ \pm}
\end{aligned}
$$

exist for every $f \in \mathcal{H}^{A} \otimes \mathfrak{T}$, where $L^{A}(z, \zeta), z \in \mathbb{C}_{ \pm}, \zeta \in \mathbb{C}_{+}$, is given by (4.11).

Proof. We divide the proof in several steps. (i) Let $f \in \operatorname{dom}\left(I_{\mathcal{H}^{A}} \otimes T\right)$. Then

$$
\int_{\mathbb{R}} \lambda^{2} d\left\|\widehat{E}_{T}(\lambda) f\right\|^{2}<\infty
$$

Note that in accordance with (2.8),

$$
\begin{aligned}
& \left\|\left(\operatorname{Im}\left(M^{A}(i-\lambda)\right)\right)^{1 / 2} \otimes I_{\mathcal{T}}\right\|=O(|\lambda|) \quad \text { and } \\
& \left\|\left(\operatorname{Im}\left(M^{A}(i-\lambda)\right)\right)^{-1 / 2} \otimes I_{\mathcal{T}}\right\|=O(|\lambda|) \quad \text { as } \quad \lambda \rightarrow \infty .
\end{aligned}
$$

Therefore the convergence of the integrals in (4.16) and (4.17) is immediate from Proposition 3.7 with $\alpha=1$.

(ii) To prove (4.18) it suffices to show that

$$
\left\|\left(\operatorname{Im}\left(M^{A}(i-\lambda)\right)\right)^{-1 / 2} \operatorname{Re}\left(M^{A}(i-\lambda)\right)\right\|=O(|\lambda|) \quad \text { as } \quad \lambda \rightarrow \infty .
$$


Noting that

$$
\begin{aligned}
& \left(\operatorname{Im}\left(M^{A}(i-\lambda)\right)^{-1 / 2} M^{A}(i-\lambda)=\right. \\
& \quad\left(\operatorname{Im}\left(M^{A}(i-\lambda)\right)^{-1 / 2} \operatorname{Re}\left(M^{A}(i-\lambda)\right)+i\left(\operatorname{Im}\left(M^{A}(i-\lambda)\right)^{1 / 2}\right.\right.
\end{aligned}
$$

and taking estimate (2.8) into account one concludes that the required estimate (4.21) is equivalent to the following one

$$
\|\left(\operatorname{Im}\left(M^{A}(i-\lambda)\right)^{-1 / 2} M^{A}(i-\lambda) \|=O(|\lambda|) \quad \text { as } \quad \lambda \rightarrow \infty .\right.
$$

Further, in accordance with (2.5)

$$
\begin{aligned}
\operatorname{Im}\left(M^{A}(i-\lambda)\right) & =-\operatorname{Im}\left(M^{A}(-i-\lambda)\right)=\gamma^{A}(-i-\lambda)^{*} \gamma^{A}(-i-\lambda) \\
& =\gamma^{A}(i-\lambda)^{*} \gamma^{A}(i-\lambda), \quad \lambda \in \mathbb{R} .
\end{aligned}
$$

Hence there exists a family of isometries $V(\lambda \pm i)$ mapping $\mathcal{H}^{A}$ onto $\mathcal{N}_{A}( \pm i-\lambda)=$ $\operatorname{ker}\left(A^{*}+\lambda \mp i\right)$ and such that

$$
V(\lambda \pm i)\left(\operatorname{Im}\left(M^{A}(i-\lambda)\right)^{1 / 2}=\gamma^{A}( \pm i-\lambda), \quad \lambda \in \mathbb{R} .\right.
$$

Using (2.5), we get

$$
\begin{aligned}
M^{A}(i-\lambda)-M^{A}(i)^{*} & =(2 i-\lambda) \gamma^{A}(-i-\lambda)^{*} \gamma^{A}(-i) \\
& =(2 i-\lambda)\left(\operatorname{Im}\left(M^{A}(i-\lambda)\right)^{1 / 2} V(\lambda)^{*} \gamma^{A}(-i) .\right.
\end{aligned}
$$

Thus

$$
\begin{aligned}
& \left(\operatorname{Im}\left(M^{A}(i-\lambda)\right)^{-1 / 2} M^{A}(i-\lambda)\right. \\
& \quad=(2 i-\lambda) V(\lambda)^{*} \gamma^{A}(-i)+\left(\operatorname{Im}\left(M^{A}(i-\lambda)\right)^{-1 / 2} M^{A}(-i) .\right.
\end{aligned}
$$

Combining this relation with estimate (2.8) yields (4.22) as well as

$$
\left\|\left(\operatorname{Im}\left(M^{A}(i-\lambda)\right)\right)^{-1 / 2} \operatorname{Re}\left(M^{A}(i-\lambda)\right)\right\|=O(|\lambda|) .
$$

To prove (4.18) it remains to apply Proposition 3.7 with $\alpha=1$.

(iii) To prove the convergence of the integral (4.19) it suffices to show that

$$
\| \gamma^{A}(z-\lambda)\left(\operatorname{Im}\left(M^{A}(i-\lambda)\right)^{-1 / 2} \| \leq \varkappa(z), \quad \lambda \in \mathbb{R} .\right.
$$

with some positive constant $\varkappa(z)>0$. In accordance with (2.4)

$$
\gamma^{A}(z-\lambda)=\left(A_{0}+\lambda-i\right)\left(A_{0}+\lambda-z\right)^{-1} \gamma^{A}(i-\lambda), \quad z \in \mathbb{C}_{ \pm}, \quad \lambda \in \mathbb{R} .
$$

Moreover, it follows from (4.23) that

$$
\left(\operatorname{Im}\left(M^{A}(i-\lambda)\right)^{-1 / 2}=\left(\gamma^{A}(i-\lambda)\right)^{-1} V(i+\lambda), \quad \lambda \in \mathbb{R} .\right.
$$

Combining these relations yields

$$
\gamma^{A}(z-\lambda)\left(\operatorname{Im}\left(M^{A}(i-\lambda)\right)^{-1 / 2}=\left(A_{0}+\lambda-i\right)\left(A_{0}+\lambda-z\right)^{-1} V(i+\lambda),\right.
$$


$z \in \mathbb{C}_{ \pm}, \lambda \in \mathbb{R}$. On the other hand

$\left\|\left(A_{0}+\lambda-i\right)\left(A_{0}+\lambda-z\right)^{-1}\right\|=\left\|I+(z-i)\left(A_{0}+\lambda-z\right)^{-1}\right\| \leq 1+\frac{|z-i|}{|\operatorname{Im} z|}=: \varkappa(z)$.

Combining this estimate with identity (4.25) we arrive at the estimate (4.24). Proposition 3.7 with $\alpha=1$ completes the proof.

(iv) To prove the existence of the integral (4.20) it suffices to show that for each fixed $z \in \mathbb{C}_{ \pm}$

$$
\left\|L^{A}(z-\lambda, i-\lambda)\right\|=O(1) \quad \text { as } \quad \lambda \rightarrow \infty,
$$

and apply Proposition 3.7. It follows from (4.11) and identity (2.5) that

$$
\begin{aligned}
& L^{A}(z-\lambda, i-\lambda) \\
& =\frac{1}{\sqrt{\operatorname{Im}\left(M^{A}(i-\lambda)\right)}}\left(M^{A}(z-\lambda)-M^{A}(i-\lambda)\right) \frac{1}{\sqrt{\operatorname{Im}\left(M^{A}(i-\lambda)\right)}}+i I_{\mathcal{H}} \\
& =(z-i) \frac{1}{\sqrt{\operatorname{Im}\left(M^{A}(i-\lambda)\right)}} \gamma^{A}(-i-\lambda)^{*} \gamma^{A}(z-\lambda) \frac{1}{\sqrt{\operatorname{Im}\left(M^{A}(i-\lambda)\right)}}+i I_{\mathcal{H}},
\end{aligned}
$$

$z \in \mathbb{C}_{ \pm}, \lambda \in \mathbb{R}$. Inserting in this identity instead of $\gamma^{A}(-i-\lambda)^{*}$ its expression from (4.23) one gets

$$
\begin{aligned}
& L^{A}(z-\lambda, i-\lambda) \\
& \quad=(z-i) V(\lambda-i)^{*} \gamma^{A}(z-\lambda) \frac{1}{\sqrt{\operatorname{Im}\left(M^{A}(i-\lambda)\right)}}+i I_{\mathcal{H}} .
\end{aligned}
$$

Finally, combining this identity with (4.24) implies (4.26).

Remark 4.7 Combining estimates (4.21) and (2.8) we obtain

$$
\begin{aligned}
& \left\|\operatorname{Re}\left(M^{A}(i-\lambda)\right)\right\| \text { le }\left\|\left(\operatorname{Im}\left(M^{A}(i-\lambda)\right)\right)^{1 / 2}\right\| \times \\
& \quad \times\left\|\left(\operatorname{Im}\left(M^{A}(i-\lambda)\right)\right)^{-1 / 2} \operatorname{Re}\left(M^{A}(i-\lambda)\right)\right\|=O\left(|\lambda|^{2}\right) .
\end{aligned}
$$

as $\lambda \rightarrow \infty$. Simple examples show that even for a scalar Nevanlinna function $f \in R[\mathbb{C}]$ the function $\left\|(\operatorname{Im}(f(i-\lambda)))^{-1 / 2} \operatorname{Re}(f(i-\lambda))\right\|$ is not necessarily bounded.

Theorem 4.8 Let $\Pi_{A}=\left\{\mathcal{H}^{A}, \Gamma_{0}^{A}, \Gamma_{1}^{A}\right\}$ be a boundary triplet for $A^{*}$, let $M^{A}(\cdot)$ and $\gamma^{A}(\cdot)$ be the corresponding Weyl function and $\gamma$-field, respectively. Let also $T=T^{*} \in \mathcal{C}(\mathfrak{T}) \backslash \mathcal{B}(\mathfrak{T})$ and $S:=A \otimes I_{\mathfrak{T}}+I_{\mathfrak{H}} \otimes T$. Then:

(i) There exists a normalized boundary triplet $\widetilde{\Pi}_{S}=\left\{\widetilde{\mathcal{H}}^{S}, \widetilde{\Gamma}_{0}^{S}, \widetilde{\Gamma}_{1}^{S}\right\}$ for $S^{*}$ such that $\widetilde{\mathcal{H}}^{S}:=\mathcal{H}^{A} \otimes \mathfrak{T}$ and $S_{0}:=S^{*} \uparrow \operatorname{ker}\left(\widetilde{\Gamma}_{0}^{S}\right)=A_{0} \otimes I_{\mathfrak{T}}+I_{\mathfrak{H}} \otimes T$, and for any 
$f \in \mathfrak{D}:=\operatorname{dom}\left(S^{*}\right) \cap \operatorname{dom}\left(I_{\mathfrak{H}} \otimes T\right)\left(\subseteq \operatorname{dom}\left(S^{*}\right)\right)$

$$
\begin{aligned}
\widetilde{\Gamma}_{0}^{S} f & :=\left(\int_{\mathbb{R}} \widehat{E}_{T}(d \lambda) \sqrt{\operatorname{Im}\left(M^{A}(i-\lambda)\right)} \otimes I_{\mathfrak{T}}\right) \cdot\left(\Gamma_{0}^{A} \widehat{\otimes} I_{\mathfrak{T}}\right) f \\
\widetilde{\Gamma}_{1}^{S} f & :=\left(\int_{\mathbb{R}} \widehat{E}_{T}(d \lambda) \frac{1}{\sqrt{\operatorname{Im}\left(M^{A}(i-\lambda)\right)}} \otimes I_{\mathfrak{T}}\right) \cdot\left(\Gamma_{1}^{A} \widehat{\otimes} I_{\mathfrak{T}}\right) f \\
& -\left(\int_{\mathbb{R}} \widehat{E}_{T}(d \lambda) \frac{1}{\sqrt{\operatorname{Im}\left(M^{A}(i-\lambda)\right)}} \operatorname{Re}\left(M^{A}(i-\lambda)\right) \otimes I_{\mathfrak{T}}\right) \cdot\left(\Gamma_{0}^{A} \widehat{\otimes} I_{\mathfrak{T}}\right) f .
\end{aligned}
$$

(ii) The $\gamma$-field $\widetilde{\gamma}^{S}(\cdot)$ and Weyl function $\widetilde{M}^{S}(\cdot)$ corresponding to the triplet $\widetilde{\Pi}_{S}$ are given by

$$
\widetilde{\gamma}^{S}(z)=G(z) \quad \text { and } \quad \widetilde{M}^{S}(z)=M(z), \quad z \in \mathbb{C}_{ \pm},
$$

where $G(\cdot)$ and $M(\cdot)$ are defined by (4.19) and (4.20), respectively.

(iii) If $M^{A}(\cdot)$ is of scalar type, i.e. $M^{A}(\cdot)=m^{A}(\cdot) I_{\mathcal{H}^{A}}$, then representation (4.12) remains true.

Proof. (i) Clearly, $f \in \operatorname{dom}\left(A^{*} \otimes I_{\mathfrak{T}}\right)$. Let $\Delta_{n}:=[n, n+1), n \in \mathbb{Z}$. We set $\mathfrak{T}_{n}:=E_{T}\left(\Delta_{n}\right) \mathfrak{T}$ and $T_{n}=T E_{T}\left(\Delta_{n}\right), n \in \mathbb{Z}$. Notice that $\mathfrak{T}=\bigoplus_{n \in \mathbb{Z}} \mathfrak{T}_{n}$ and $T=\bigoplus_{n \in \mathbb{Z}} T_{n}$. Let also $R_{S_{n}}:=\sqrt{\operatorname{Im}\left(M^{S_{n}}(i)\right)}$ and $Q_{S_{n}}:=\operatorname{Re}\left(M^{S_{n}}(i)\right), n \in \mathbb{Z}$. Then, by Proposition 4.4, a triplet $\widetilde{\Pi}_{S_{n}}=\left\{\mathcal{H}^{S_{n}}, \widetilde{\Gamma}_{0}^{S_{n}}, \widetilde{\Gamma}_{1}^{S_{n}}\right\}$ with

$$
\begin{aligned}
& \mathcal{H}^{S_{n}}:=\mathcal{H}^{A} \otimes \mathfrak{T}_{n}, \quad \widetilde{\Gamma}_{0}^{S_{n}}=R_{S_{n}}\left(\Gamma_{0}^{A} \widehat{\otimes} I_{\mathfrak{T}_{n}}\right), \quad \text { and } \\
& \quad \widetilde{\Gamma}_{1}^{S_{n}}=R_{S_{n}}^{-1}\left(\Gamma_{1}^{S_{n}}-Q_{S_{n}} \Gamma_{0}^{S_{n}}\right)=R_{S_{n}}^{-1} \Gamma_{1}^{S_{n}}-R_{S_{n}}^{-1} Q_{S_{n}} \Gamma_{0}^{S_{n}},
\end{aligned}
$$

is a boundary triplet for $S_{n}^{*}$ for each $n \in \mathbb{Z}$. In turn, Theorem 2.7 ensures that the direct sum $\widetilde{\Pi}_{S}:=\bigoplus_{n \in \mathbb{Z}} \widetilde{\Pi}_{S_{n}}=\left\{\widetilde{\mathcal{H}}^{S}, \widetilde{\Gamma}_{0}^{S}, \widetilde{\Gamma}_{1}^{S}\right\}$ of boundary triplets is an ordinary (normalized) boundary triplet for $S^{*}=\bigoplus_{n \in \mathbb{Z}} S_{n}^{*}$.

Setting $R:=\bigoplus_{n} R_{S_{n}}$, applying formula (4.14) and noting that, by Lemma 4.6. the improper spectral integral (4.16) exists one gets that for any $h=$ $\bigoplus_{n} h_{n} \in \operatorname{dom}\left(I_{\mathcal{H}^{\mathrm{A}}} \otimes T\right)=\oplus_{n} \operatorname{dom}\left(I_{\mathcal{H}^{\mathrm{A}}} \otimes T_{n}\right)$

$$
\begin{aligned}
R h & =\bigoplus_{n \in \mathbb{Z}} R_{S_{n}} h_{n}=\bigoplus_{n \in \mathbb{Z}} \sqrt{\operatorname{Im}\left(M^{S_{n}}(i)\right)} h_{n} \\
& =\bigoplus_{n \in \mathbb{Z}} \int_{[n, n+1)} \widehat{E}_{T_{n}}(\lambda)\left(\sqrt{\operatorname{Im}\left(M^{A}(i-\lambda)\right.} \otimes I_{\mathfrak{T}_{n}}\right) h_{n} \\
& =\operatorname{s-}_{\substack{p \rightarrow+\infty \\
q \rightarrow-\infty}} \int_{[q, p)} \widehat{E}_{T}(\lambda)\left(\sqrt{\operatorname{Im}\left(M^{A}(i-\lambda)\right.} \otimes I_{\mathfrak{T}}\right) h \\
& =\int_{\mathbb{R}} \widehat{E}_{T}(\lambda)\left(\sqrt{\operatorname{Im}\left(M^{A}(i-\lambda)\right.} \otimes I_{\mathfrak{T}}\right)=G_{0} h .
\end{aligned}
$$


Note that applying formula (4.14) we have replaced the integral $\int_{[n, n+1]}$ by $\int_{[n, n+1)}$. The latter is possible since $n+1 \notin \sigma_{p}\left(T_{n}\right)$ for each $n \in \mathbb{Z}$.

Next, similarly to (4.29) and using the convergence of the improper spectral integral (4.17) one gets from (4.14)

$$
\begin{aligned}
R^{-1} h & =\bigoplus_{n \in \mathbb{Z}} R_{S_{n}}^{-1} h_{n}=\bigoplus_{n \in \mathbb{Z}}\left(\sqrt{\operatorname{Im}\left(M^{S_{n}}(i)\right)}\right)^{-1} h_{n} \\
& =\int_{\mathbb{R}} \widehat{E}_{T}(d \lambda)\left(\frac{1}{\sqrt{\operatorname{Im}\left(M^{A}(i-\lambda)\right)}} \otimes I_{\mathfrak{T}}\right) h=G_{1} h .
\end{aligned}
$$

Further, setting $Q:=\bigoplus_{n} Q_{S_{n}}:=\bigoplus_{n} \operatorname{Re}\left(M^{S_{n}}(i)\right)$, applying formula (4.15) with $\Delta_{n}$ in place of $\Delta$, and noting that by Lemma 4.6 the improper spectral integral (4.18) exists, we derive

$$
\begin{aligned}
R^{-1} Q h & =\bigoplus_{n \in \mathbb{Z}} R_{S_{n}}^{-1} \operatorname{Re}\left(M^{S_{n}}(i)\right) h_{n} \\
= & \left.\bigoplus_{n \in \mathbb{Z}} \int_{[n, n+1)} \widehat{E}_{T_{n}}(\lambda)\left(\frac{1}{\sqrt{\operatorname{Im}\left(M^{A}(i-\lambda)\right)}} \operatorname{Re}\left(M^{A}(i-\lambda)\right) \otimes I_{\mathfrak{T}_{n}}\right)\right) h_{n} \\
= & \left.\int_{\mathbb{R}} \widehat{E}_{T}(\lambda)\left(\frac{1}{\sqrt{\operatorname{Im}\left(M^{A}(i-\lambda)\right)}} \operatorname{Re}\left(M^{A}(i-\lambda)\right) \otimes I_{\mathfrak{T}}\right)\right) h=G_{2} h .
\end{aligned}
$$

Further, let $f=\left\{f_{n}\right\}_{n \in \mathbb{Z}} \in \mathfrak{D} \subseteq \operatorname{dom}\left(A^{*} \otimes I_{\mathfrak{T}}\right), f_{n} \in \mathfrak{H}_{A} \otimes \mathfrak{T}_{n}, n \in \mathbb{Z}$. Note that $f \in \operatorname{dom}\left(\Gamma_{0}^{A} \widehat{\otimes} I_{\mathfrak{T}}\right) \cap \operatorname{dom}\left(\Gamma_{1}^{A} \widehat{\otimes} I_{\mathfrak{T}}\right)$ because $f \in \operatorname{dom}\left(A^{*} \otimes I_{\mathfrak{T}}\right)$. Hence

$$
\left(\Gamma_{0}^{A} \widehat{\otimes} I_{\mathfrak{T}}\right) f=\bigoplus_{n \in \mathbb{Z}}\left(\Gamma_{0}^{A} \widehat{\otimes} I_{\mathfrak{T}_{n}}\right) f_{n} \quad \text { and } \quad\left(\Gamma_{1}^{A} \widehat{\otimes} I_{\mathfrak{T}}\right) f=\bigoplus_{n \in \mathbb{Z}}\left(\Gamma_{1}^{A} \widehat{\otimes} I_{\mathfrak{T}_{n}}\right) f_{n} .
$$

On the other hand, by Theorem 2.7 (see formula (2.13) )

$$
\widetilde{\Gamma}_{0}^{S} f=R\left(\Gamma_{1}^{A} \widehat{\otimes} I_{\mathfrak{T}}\right) f \quad \text { and } \quad \widetilde{\Gamma}_{1}^{S} f=R^{-1}\left(\Gamma_{1}^{A} \widehat{\otimes} I_{\mathfrak{T}}\right) f+R^{-1} Q\left(\Gamma_{0}^{A} \widehat{\otimes} I_{\mathfrak{T}}\right) f,
$$

$f \in \mathfrak{D}$. Inserting in these relations instead of $R, R^{-1}$, and $R^{-1} Q$ their expressions from (4.29), (4.30), and (4.31), one arrives at formulas (4.27).

(ii) In accordance with Proposition 4.4(ii) the $\gamma$-field and Weyl function corresponding to the triplet $\widetilde{\Pi}_{S_{n}}=\left\{\mathcal{H}^{S_{n}}, \widetilde{\Gamma}_{0}^{S_{n}}, \widetilde{\Gamma}_{1}^{S_{n}}\right\}$ are given by

$$
\widetilde{\gamma}^{S_{n}}(z)=\int_{[n, n+1)}\left(\gamma^{A}(z-\lambda) \frac{1}{\sqrt{\operatorname{Im}\left(M^{A}(i-\lambda)\right)}} \otimes I_{\mathfrak{T}_{n}}\right) \widehat{E}_{T_{n}}(d \lambda), \quad z \in \mathbb{C}_{ \pm},
$$

and

$$
\begin{aligned}
\widetilde{M}^{S_{n}}(z) & =\int_{[n, n+1)}\left(L^{A}(z-\lambda, i-\lambda) \otimes I_{\mathfrak{T}_{n}}\right) \widehat{E}_{T_{n}}(d \lambda) \\
& =\int_{[n, n+1)} \widehat{E}_{T_{n}}(d \lambda)\left(L^{A}(z-\lambda, i-\lambda) \otimes I_{\mathfrak{T}_{n}}\right), \quad z \in \mathbb{C}_{ \pm},
\end{aligned}
$$


respectively. Here $L^{A}(z, \zeta)$ is given by (4.11). Further, applying Theorem 2.7 (see formula (2.14) ) and taking into account formulas (4.19) and (4.20), we arrive at (4.28).

(iii) This statement is now immediate from formula (4.12) and representation $T=\bigoplus_{n \in \mathbb{Z}} T_{n}$ with $T_{n} \in \mathcal{B}\left(\mathfrak{T}_{n}\right)$.

\section{Remark 4.9}

(i) If $T$ is pure point, $\sigma(T)=\sigma_{p p}(T)=\left\{\lambda_{k}\right\}_{k \in \mathbb{Z}}$, then the boundary space $\mathcal{H}^{S}$ admits the representation $\mathcal{H}^{S}=\bigoplus_{k \in \mathbb{Z}} \mathcal{H}_{k}$, where $\mathcal{H}_{k}=\mathcal{H}^{A} \otimes \mathfrak{T}_{k}$ and $\mathfrak{T}_{k}$ is the eigenspace which corresponds to $\lambda_{k}$. One easily checks that the Weyl function admits the representation

$$
M^{S}(z)=\bigoplus_{k \in \mathbb{Z}}\left(L\left(z-\lambda_{k}, i-\lambda_{k}\right) \otimes I_{\mathfrak{T}_{k}}\right), \quad z \in \mathbb{C}_{ \pm} .
$$

(ii) The set $\mathfrak{D}:=\operatorname{dom}\left(S^{*}\right) \cap \operatorname{dom}\left(I_{\mathfrak{H}_{A}} \otimes T\right) \subseteq \operatorname{dom}\left(S^{*}\right)$ is a core for $S^{*}$. Equivalently this means that $\mathfrak{D}$ regarded as a subset $\widehat{\mathfrak{D}}$ of $\mathfrak{H}_{+}\left(S^{*}\right)$ is dense in the Hilbert space $\mathfrak{H}_{+}\left(S^{*}\right)$. Let $J_{S^{*}}: \mathfrak{H}_{+}\left(S^{*}\right) \longrightarrow \mathfrak{H}_{S}=\mathfrak{H}_{A} \otimes \mathfrak{T}$ be the embedding operator. We set $\widehat{\Gamma}_{j}^{S}:=\Gamma_{j}^{S} J_{S^{*}}: \mathfrak{H}_{+}\left(S^{*}\right) \longrightarrow \mathcal{H}^{S}, j \in\{0,1\}$. The operator $\widehat{\Gamma}_{j}^{S}$, $j \in\{0,1\}$, is bounded. Hence

$$
\widehat{\Gamma}_{j}^{S}=\overline{\Gamma_{j}^{S} J_{S^{*}} \uparrow \widehat{\mathfrak{D}}}, \quad j \in\{0,1\} .
$$

In other words, the closure of the operator $\Gamma_{j}^{S} \uparrow \mathfrak{D}, j \in\{0,1\}$, with respect to the topology of $\mathfrak{H}_{+}\left(S^{*}\right)$ gives $\Gamma_{j}^{S}, j \in\{0,1\}$.

Remark 4.10 The case of a scalar type Weyl function can be slightly extended. Let us assume that there is a boundary triplet $\Pi_{A}=\left\{\mathcal{H}^{A}, \Gamma_{0}^{A}, \Gamma_{1}^{A}\right\}$ of $A^{*}$ such that $\mathcal{H}^{A}=\bigoplus_{k=1}^{n(A)} \mathcal{H}_{k}^{A}, \mathcal{H}_{k}^{A}:=\mathbb{C}, n(A):=n_{ \pm}(A)$. With respect to this decomposition we suppose that the Weyl function $M^{A}(\cdot)$ is diagonal, that is, it admits the representation

$$
\begin{aligned}
& M^{A}(z)=\operatorname{diag}\left(m_{1}(z), m_{2}(z), \ldots, m_{n(A)}(z)\right)
\end{aligned}
$$

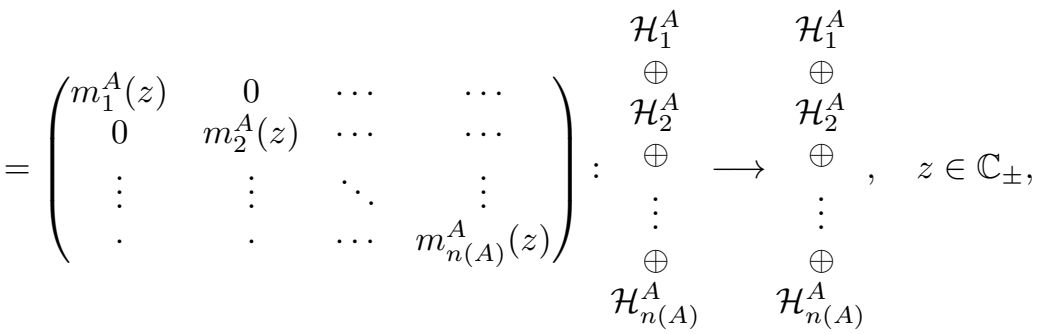

where $m_{k}(\cdot), k=1,2, \ldots, n(A)$, are scalar Nevanlinna functions. If the Weyl function of a boundary triplet has this structure, then it is called of quasi scalar type. We are going to compute the boundary triplet $\Pi_{S}$ as well $\gamma$-field $\gamma^{S}(\cdot)$ and Weyl function $M^{S}(\cdot)$ for the quasi scalar type case. We set

$$
\Gamma_{j k}^{A}:=P_{\mathcal{H}_{k}^{A}}^{\mathcal{H}^{A}} \Gamma_{j}: \operatorname{dom}\left(A^{*}\right) \longrightarrow \mathcal{H}_{k}^{A}, \quad j=0,1, \quad k=1,2, \ldots, n(A) .
$$


Obviously, we have

$$
\Gamma_{1 k}^{A} f_{z}=m_{k}(z) \Gamma_{0 k}^{A} f_{z}, \quad f_{z} \in \operatorname{ker}\left(A^{*}-z\right), \quad k=1,2, \ldots, n(A) .
$$

Let us introduce the operator $\Gamma_{j k}^{A} \widehat{\otimes} I_{\mathfrak{T}}: \operatorname{dom}\left(A^{*} \otimes I_{\mathfrak{T}}\right) \longrightarrow \mathcal{H}_{k}^{S}:=\mathcal{H}_{k}^{A} \otimes \mathfrak{T}=\mathfrak{T}$, $j=0,1, k=1,2, \ldots, n(A)$. Notice that

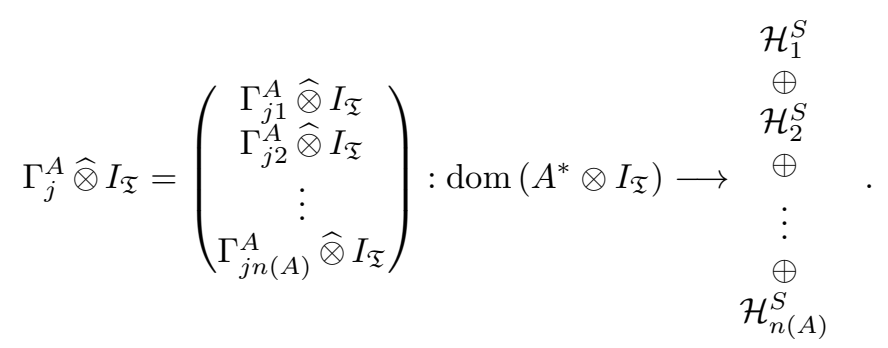

Notice that $\mathcal{H}^{S}=\mathcal{H}^{A} \otimes \mathfrak{T}=\bigoplus_{k=1}^{n(A)} \mathcal{H}_{k}^{S}$. Setting $\Gamma_{j k}^{S}:=P_{\mathcal{H}_{k}^{S}}^{\mathcal{H}^{S}} \Gamma_{j}^{S}, j \in\{0,1\}$, $k \in\{1,2, \ldots, n(A)\}$, we get $\Gamma_{j}^{S}=\left(\Gamma_{j 1}^{S}, \Gamma_{j 2}^{S}, \ldots, \Gamma_{j n(A)}^{S}\right)^{t}, j \in\{0,1\}$. Using (4.27) we get

$$
\begin{aligned}
\Gamma_{0 k}^{S} f & =\sqrt{\operatorname{Im}\left(m_{k}(i-T)\right)}\left(\Gamma_{0 k}^{A} \widehat{\otimes} I_{\mathfrak{T}}\right) f \\
\Gamma_{1 k}^{S} f & =\frac{1}{\sqrt{\operatorname{Im}\left(m_{k}(i-T)\right)}}\left(\Gamma_{1 k}^{A} \widehat{\otimes} I_{\mathfrak{T}}-\operatorname{Re}\left(m_{k}(i-T)\right)\left(\Gamma_{0 k}^{A} \widehat{\otimes} I_{\mathfrak{T}}\right)\right) f,
\end{aligned}
$$

$f \in \operatorname{dom}\left(A^{*} \otimes I_{\mathfrak{T}}\right) \cap \operatorname{dom}\left(I_{\mathfrak{H}_{A}} \otimes T\right), k \in\{1,2, \ldots, n(A)\}$.

To compute the $\gamma$-field we set

$$
\gamma_{k}^{A}(z):=\gamma^{A}(z)\left\lceil\mathcal{H}_{k}^{A}, \quad \gamma^{A}(z)=\left(\gamma_{1}^{A}(z), \gamma_{2}^{A}(z), \ldots, \gamma_{n(A)}^{A}(z)\right),\right.
$$

$z \in \mathbb{C}_{ \pm}$, and

$$
\gamma_{k}^{S}(\cdot)=\gamma^{S}(\cdot) \uparrow \mathcal{H}_{k}^{S}, \quad \gamma^{S}(z)=\left(\gamma_{1}^{S}(z), \gamma_{2}^{S}(z), \ldots, \gamma_{n(A)}^{S}(z)\right),
$$

$z \in \mathbb{C}_{ \pm}$, where $\mathcal{H}_{k}^{S}:=\mathcal{H}_{k}^{A} \otimes \mathfrak{T}=\mathfrak{T}, k \in\{1,2, \ldots, n(A)\}$. From (4.19) we find

$$
\gamma_{k}^{S}(z)=\gamma_{k}^{A}(z-T) \frac{1}{\sqrt{\operatorname{Im}\left(m_{k}(i-T)\right)}}, \quad z \in \mathbb{C}_{ \pm}, \quad k \in\{1,2, \ldots, n(A)\} .
$$

Finally, the Weyl function takes the form

$$
\begin{aligned}
& M^{S}(z)= \\
& \operatorname{diag}\left(\frac{m_{1}^{A}(z-T)-\operatorname{Re}\left(m_{1}(i-T)\right)}{\operatorname{Im}\left(m_{1}(i-T)\right)}, \ldots, \frac{m_{n(A)}^{A}(z-T)-\operatorname{Re}\left(m_{n(A}(i-T)\right)}{\operatorname{Im}\left(m_{n(A)}(i-T)\right)}\right) \\
& z \in \mathbb{C}_{ \pm} .
\end{aligned}
$$




\section{Sums of tensor products with non- negative summands}

\subsection{Boundary triplets in the case of non-negative opera- tors $A$ and $T$}

Here we complete previous results assuming the operators $A$ and $T$ to be nonnegative. We denote by $\widehat{A}_{\mathrm{F}}$ and $\widehat{A}_{\mathrm{K}}$ the Friedrich's and Krein's extension of $A$, respectively.

Theorem 5.1 Let $A$ be a non-negative symmetric operator in $\mathfrak{H}$ and let $\Pi_{A}=$ $\left\{\mathcal{H}^{A}, \Gamma_{0}^{A}, \Gamma_{1}^{A}\right\}$ be a boundary triplet for $A^{*}$ such that $A_{0}:=A^{*} \uparrow \operatorname{ker}\left(\Gamma_{0}^{A}\right)=$ $\widehat{A}_{F}$. Let also $M^{A}(\cdot)$ and $\gamma^{A}(\cdot)$ be the corresponding Weyl function and $\gamma$-field, respectively. Let also $T=T^{*} \in \mathcal{B}(\mathfrak{T}), T \geq 0$ and let $S=A \otimes I_{\mathfrak{T}}+I_{\mathfrak{H}} \otimes T$. Finally, let $\widehat{E}_{T}(\cdot):=I_{\mathcal{H}^{A}} \otimes E_{T}(\cdot)$, where $E_{T}(\cdot)$ is the spectral measure of $T$. Then:

(i) $\Pi_{S}=\left\{\mathcal{H}^{S}, \Gamma_{0}^{S}, \Gamma_{1}^{S}\right\}:=\Pi_{A} \widehat{\otimes} I_{\mathfrak{T}}:=\left\{\mathcal{H}^{A} \otimes \mathfrak{T}, \Gamma_{0}^{A} \widehat{\otimes} I_{\mathfrak{T}}, \Gamma_{1}^{A} \widehat{\otimes} I_{\mathfrak{T}}\right\}$ is a boundary triplet for $S^{*}$ such that

$$
S_{0}:=S^{*} \uparrow \operatorname{ker}\left(\Gamma_{0}^{S}\right)=\widehat{S}_{F}=\widehat{A}_{F} \otimes I_{\mathfrak{T}}+I_{\mathfrak{H}} \otimes T .
$$

(ii) The $\gamma$-field $\gamma^{S}(\cdot)$ and Weyl function $M^{S}(\cdot)$ of $\Pi_{S}$ admit the following representations

$$
\gamma^{S}(z)=\int_{\Delta}\left(\gamma^{A}(z-\lambda) \otimes I_{\mathfrak{T}}\right) \widehat{E}_{T}(d \lambda), \quad z \in \mathbb{C} \backslash \Delta
$$

and

$$
\begin{aligned}
M^{S}(z) & =\int_{\Delta} \widehat{E}_{T}(d \lambda)\left(M^{A}(z-\lambda) \otimes I_{\mathfrak{T}}\right) \\
& =\int_{\Delta}\left(M^{A}(z-\lambda) \otimes I_{\mathfrak{T}}\right) \widehat{E}_{T}(d \lambda), \quad z \in \mathbb{C} \backslash \Delta,
\end{aligned}
$$

where $\Delta$ is the smallest closed interval containing the spectrum $\sigma(T)$.

(iii) If the Weyl function $M^{A}(\cdot)$ is of scalar type, $M^{A}(\cdot)=m^{A}(\cdot) I_{\mathcal{H}^{A}}$, then

$$
M^{S}(z)=I_{\mathcal{H}^{A}} \otimes m^{A}(z-T), \quad z \in \mathbb{C}_{ \pm} .
$$

In particular, the latter holds whenever $n_{ \pm}(A)=1$.

Proof. (i) It is immediate from the definition that $S_{0}=S^{*} \uparrow \operatorname{ker}\left(\Gamma_{0}^{S}\right)=$ $A_{0} \otimes I_{\mathfrak{T}}+I_{\mathfrak{H}} \otimes T$. It remains to apply Proposition 5.6.

Statements (ii) and (iii) are immediate from Theorem 4.1 
Lemma 5.2 Let $A$ be a densely defined closed non-negative symmetric operator in $\mathfrak{H}$ and let $\Pi_{A}=\left\{\mathcal{H}^{A}, \Gamma_{0}^{A}, \Gamma_{1}^{A}\right\}$ be a boundary triplet for $A^{*}$ and let $A_{0} \geq$ 0 . Let also $M^{A}(\cdot)$ and $\gamma^{A}(\cdot)$ be the corresponding Weyl function and $\gamma$-field, respectively. Further, let $T$ be a non-negative self-adjoint operator on $\mathfrak{T}$, let $E_{T}(\cdot)$ be its spectral measure, and let $\widehat{E}_{T}(\cdot):=I_{\mathcal{H}^{A}} \otimes E_{T}(\cdot)$. Then the following improper spectral integrals

$$
\begin{aligned}
G_{0}^{+} f:=\int_{\mathbb{R}_{+}} \widehat{E}_{T}(d \lambda)\left(\sqrt{\left(\left(M^{A}\right)^{\prime}(a-\lambda)\right)} \otimes I_{\mathfrak{T}}\right) f, \quad a<0, \\
G_{1}^{+} f:=\int_{\mathbb{R}_{+}} \widehat{E}_{T}(d \lambda)\left(\frac{1}{\sqrt{\left(\left(M^{A}\right)^{\prime}(a-\lambda)\right)}} \otimes I_{\mathfrak{T}}\right) f, \quad a<0, \\
G_{2}^{+} f:=\int_{\mathbb{R}_{+}} \widehat{E}_{T}(d \lambda)\left(\frac{1}{\sqrt{\left(M^{A}\right)^{\prime}(a-\lambda)}} M^{A}(a-\lambda) \otimes I_{\mathfrak{T}}\right) f, \quad a<0 .
\end{aligned}
$$

exist for each $f \in \operatorname{dom}\left(I_{\mathcal{H}^{A}} \otimes T\right)$. Moreover, the following improper spectral integrals

$$
G(z) f:=\int_{\mathbb{R}_{+}}\left(\gamma^{A}(z-\lambda) \frac{1}{\sqrt{\left(M^{A}\right)^{\prime}(a-\lambda)}} \otimes I_{\mathfrak{T}}\right) \widehat{E}_{T}(d \lambda) f,
$$

$z \in \mathbb{C} \backslash \mathbb{R}_{+}, a<0$, and

$$
\begin{aligned}
M(z) f & :=\int_{\mathbb{R}_{+}}\left(L^{A}(z-\lambda, a-\lambda) \otimes I_{\mathfrak{T}}\right) \widehat{E}_{T}(d \lambda) f \\
& =\int_{\mathbb{R}_{+}} \widehat{E}_{T}(d \lambda)\left(L^{A}(z-\lambda, a-\lambda) \otimes I_{\mathfrak{T}}\right) f, \quad z \in \mathbb{C} \backslash \mathbb{R}_{+},
\end{aligned}
$$

converge for every $f \in \mathcal{H}^{A} \otimes \mathfrak{T}$, where

$$
L^{A}(z, a):=\frac{1}{\sqrt{\left(M^{A}\right)^{\prime}(a)}}\left(M^{A}(z)-M^{A}(a)\right) \frac{1}{\sqrt{\left(M^{A}\right)^{\prime}(a)}},
$$

$z \in \rho\left(A_{0}\right), a \in \mathbb{R}_{-}$.

Proof. (i) First we prove the convergence of integral in (5.6). It follows from (2.5) that $\left(M^{A}\right)^{\prime}(z)=\gamma^{A}(\bar{z})^{*} \gamma^{A}(z)$. Hence

$$
\left(M^{A}\right)^{\prime}(a-\lambda)=\gamma^{A}(a-\lambda)^{*} \gamma^{A}(a-\lambda), \quad \lambda \in \mathbb{R}_{+}, \quad a<0 .
$$

This identity implies the existence of an isometry $V(a-\lambda)$ mapping $\mathcal{H}$ onto $\mathfrak{N}_{a-\lambda}(A)$ and such that

$$
V(a-\lambda) \sqrt{\left(M^{A}\right)^{\prime}(a-\lambda)}=\gamma^{A}(a-\lambda), \quad \lambda \in \mathbb{R}_{+},
$$


Further, in accordance with (2.4)

$$
\begin{aligned}
\gamma^{A}(z-\lambda) & =\left(A_{0}-a+\lambda\right)\left(A_{0}-z+\lambda\right)^{-1} \gamma^{A}(a-\lambda) \\
& =U(a-\lambda, z-\lambda) \gamma^{A}(a-\lambda),
\end{aligned}
$$

where $U(a-\lambda, z-\lambda):=\left(A_{0}-a+\lambda\right)\left(A_{0}-z+\lambda\right)^{-1} \uparrow \mathfrak{N}_{a-\lambda}(A)$. It is easily checked that $U(a-\lambda, z-\lambda)$ isomorphically maps $\mathfrak{N}_{a-\lambda}(A)$ onto $\mathfrak{N}_{z-\lambda}(A)$. Combining relation (5.11) with (5.10) yields

$$
\begin{array}{r}
\left\|\gamma^{A}(z-\lambda)\left(\left(M^{A}\right)^{\prime}(a-\lambda)\right)^{-1 / 2}\right\|=\|U(a-\lambda, z-\lambda) V(a-\lambda)\| \\
=\left\|I+(z-a)\left(A_{0}-z+\lambda\right)^{-1}\right\| \leq \begin{cases}1+|z-a| \cdot|\operatorname{Im} z|^{-1}, & z \in \mathbb{C} \backslash \mathbb{R}, \\
1+|x-a| \cdot|x|^{-1}, & x \in \mathbb{R}_{-} .\end{cases}
\end{array}
$$

Here we have taken into account that $|x| \leq|x-\lambda|=|x|+\lambda$. The latter estimate implies boundedness of the integrand in (5.6) for each $z \in \mathbb{C} \backslash \mathbb{R}_{+}$. It remains to apply Proposition 3.7 with $\alpha=0$.

(ii) Let us prove that

$$
C(z, a):=\sup _{\lambda \in \mathbb{R}_{+}}\left\|L^{A}(z-\lambda, a-\lambda)\right\|<\infty \quad \text { for each } \quad z \in \mathbb{C} \backslash \mathbb{R}_{+} \quad \text { and } \quad a<0 .
$$

Combining identity (2.5) with (5.11) yields

$$
\begin{array}{r}
M^{A}(z-\lambda)-M^{A}(a-\lambda)=(z-a) \gamma^{A}(a-\lambda)^{*} \gamma^{A}(z-\lambda) \\
=(z-a) \gamma^{A}(a-\lambda)^{*} U(a-\lambda, z-\lambda) \gamma^{A}(a-\lambda) .
\end{array}
$$

In turn, inserting this identity in (5.8) and using (5.10) one derives

$$
\begin{aligned}
& L^{A}(z-\lambda, a-\lambda)=(z-a) \frac{1}{\sqrt{\left(M^{A}\right)^{\prime}(a-\lambda)}} \gamma^{A}(a-\lambda)^{*} \times \\
& \times U(a-\lambda, z-\lambda) \gamma^{A}(a-\lambda) \frac{1}{\sqrt{\left(M^{A}\right)^{\prime}(a-\lambda)}} \\
&=(z-a) V(a-\lambda)^{*} U(a-\lambda, z-\lambda) V(a-\lambda), \quad \lambda \in \mathbb{R}_{+} .
\end{aligned}
$$

Noting that $V(a-\lambda)$ is an isometry for each $\lambda \in \mathbb{R}_{+}$and using estimate (5.12) one arrives at estimate (5.13). To prove convergence of the integral (5.7) for each $f \in \mathcal{H}^{A} \otimes \mathfrak{T}$, it remains to apply Proposition 3.7 with $\alpha=0$.

(iii) Let us prove convergence of integrals (5.3) and (5.4). Since $A_{0} \geq 0$, integral representation (2.6) implies

$$
\left(M^{A}\right)^{\prime}(a-\lambda)=\int_{\mathbb{R}_{+}} \frac{d \Sigma_{A}(t)}{(t-a+\lambda)^{2}}, \quad \lambda \in \mathbb{R}_{+}, \quad a<0 .
$$

Using this representation instead of (2.7) one proves the following analog of estimate (2.8)

$$
C_{1}\left(1+|\lambda|^{2}\right)^{-1} \operatorname{Im} M(i) \leq\left(M^{A}\right)^{\prime}(a-\lambda) \leq C_{2}\left(1+|\lambda|^{2}\right) \operatorname{Im} M(i),
$$


$\lambda \in \mathbb{R}_{+}$. Combining this estimate with inequality $\int_{\mathbb{R}_{+}} \lambda^{2} d\left\|\widehat{E}_{T}(\lambda) f\right\|^{2}<\infty$ characterizing $f \in \operatorname{dom}\left(I_{\mathcal{H}^{A}} \otimes T\right)$, and applying Proposition 3.7 with $\alpha=1$ yields convergence of both integrals (5.3) and (5.4).

(iv) Due to Proposition 3.7 (with $\alpha=1$ ) to prove (5.5) it suffices to show that

$$
\left\|\left(\left(M^{A}\right)^{\prime}(a-\lambda)\right)^{-1 / 2} M^{A}(a-\lambda)\right\|=O(|\lambda|) \quad \text { as } \quad \lambda \rightarrow \infty .
$$

In accordance with (2.5)

$$
M^{A}(a-\lambda)=M^{A}(a)-\lambda \gamma^{A}(a-\lambda)^{*} \gamma^{A}(a) .
$$

Combining this identity with (5.9) we derive

$$
\begin{aligned}
& \left\|\left(\left(M^{A}\right)^{\prime}(a-\lambda)\right)^{-1 / 2} M^{A}(a-\lambda)\right\| \\
& \quad \leq\left\|M^{A}(a)\right\| \cdot\left\|\left(\left(M^{A}\right)^{\prime}(a-\lambda)\right)^{-1 / 2}\right\|+|\lambda| \cdot\left\|V^{*}(a-\lambda) \cdot \gamma^{A}(a)\right\| .
\end{aligned}
$$

Noting that $V(a-\lambda)$ is an isometry and taking (5.16) into account we arrive at estimate (5.17).

Theorem 5.3 Let $\Pi_{A}=\left\{\mathcal{H}^{A}, \Gamma_{0}^{A}, \Gamma_{1}^{A}\right\}$ be a boundary triplet for $A^{*}, A_{0}:=A^{*} \uparrow$ $\operatorname{ker}\left(\Gamma_{0}^{A}\right)$, let $M^{A}(\cdot)$ and $\gamma^{A}(\cdot)$ be the corresponding Weyl function and $\gamma$-field, respectively. Let also $T=T^{*} \in \mathcal{C}(\mathfrak{T})$ be an unbounded self-adjoint operator in $\mathfrak{T}$ and $S:=A \otimes I_{\mathfrak{T}}+I_{\mathfrak{H}} \otimes T$. Then:

(i) There exists a boundary triplet $\widetilde{\Pi}_{S}=\left\{\widetilde{\mathcal{H}}^{S}, \widetilde{\Gamma}_{0}^{S}, \widetilde{\Gamma}_{1}^{S}\right\}$ for $S^{*}$ such that $\widetilde{\mathcal{H}}^{S}:=$ $\mathcal{H}^{A} \otimes \mathfrak{T}$ and $S_{0}:=S^{*} \uparrow \operatorname{ker}\left(\widetilde{\Gamma}_{0}^{S}\right)=A_{0} \otimes I_{\mathfrak{T}}+I_{\mathfrak{H}} \otimes T$. If $f \in \mathfrak{D}:=\operatorname{dom}\left(S^{*}\right) \cap$ $\operatorname{dom}\left(I_{\mathfrak{H}} \otimes T\right) \subseteq \operatorname{dom}\left(S^{*}\right)$, then $f \in \operatorname{dom}\left(S^{*}\right) \cap \operatorname{dom}\left(A^{*} \otimes I_{\mathfrak{T}}\right)$ and

$$
\begin{aligned}
\widetilde{\Gamma}_{0}^{S} f & :=\left(\int_{\mathbb{R}_{+}} \widehat{E}_{T}(d \lambda) \sqrt{\left.\left(M^{A}\right)^{\prime}(a-\lambda)\right)} \otimes I_{\mathfrak{T}}\right) \cdot\left(\Gamma_{0}^{A} \widehat{\otimes} I_{\mathfrak{T}}\right) f \\
\widetilde{\Gamma}_{1}^{S} f & :=\left(\int_{\mathbb{R}_{+}} \widehat{E}_{T}(d \lambda) \frac{1}{\sqrt{\left(M^{A}\right)^{\prime}(a-\lambda)}} \otimes I_{\mathfrak{T}}\right)\left(\Gamma_{1}^{A} \widehat{\otimes} I_{\mathfrak{T}}\right) f \\
& -\left(\int_{\mathbb{R}_{+}} \widehat{E}_{T}(d \lambda)\left(\frac{1}{\sqrt{\left(M^{A}\right)^{\prime}(a-\lambda)}} M^{A}(a-\lambda) \otimes I_{\mathfrak{T}}\right)\right) \cdot\left(\Gamma_{0}^{A} \widehat{\otimes} I_{\mathfrak{T}}\right) f .
\end{aligned}
$$

(ii) The $\gamma$-field $\widetilde{\gamma}^{S}(\cdot)$ and Weyl function $\widetilde{M}^{S}(\cdot)$ corresponding to $\widetilde{\Pi}_{S}$ are given by

$$
\widetilde{\gamma}^{S}(z)=G(z) \quad \text { and } \quad \widetilde{M}^{S}(z)=M(z), \quad z \in \rho\left(S_{0}\right),
$$

where $G(\cdot)$ and $M(\cdot)$ are defined by (5.6) and (5.7), respectively.

(iii) If $M^{A}(\cdot)$ is a scalar type function, i.e. $M^{A}(\cdot)=m^{A}(\cdot) I_{\mathcal{H}^{A}}$, then representation (4.12) remains true. 
Proof. (i) First we let $\Delta_{n}:=[n-1, n), \mathfrak{T}_{n}:=E_{T}\left(\Delta_{n}\right) \mathfrak{T}$, and $T_{n}=T E_{T}\left(\Delta_{n}\right)$, $n \in \mathbb{N}$. Clearly, $\mathfrak{T}=\bigoplus_{n \in \mathbb{Z}} \mathfrak{T}_{n}$ and $T=\bigoplus_{n \in \mathbb{Z}} T_{n}$. We also put $S_{n}:=A \otimes I_{\mathfrak{T}_{n}}+$ $I_{\mathfrak{H}} \otimes T_{n} \in \mathcal{C}\left(\mathfrak{H} \otimes \mathfrak{T}_{n}\right)$. Clearly, each $T_{n}$ is bounded and $\sigma\left(T_{n}\right) \subset[n-1, n]$.

By Theorem 5.1, $\Pi_{S_{n}}=\left\{\mathcal{H}^{S_{n}}, \Gamma_{0}^{S_{n}}, \Gamma_{1}^{S_{n}}\right\}:=\Pi_{A} \widehat{\otimes} I_{\mathfrak{T}_{n}}:=\left\{\mathcal{H}^{A} \otimes\right.$ $\left.\mathfrak{T}_{n}, \Gamma_{0}^{A} \widehat{\otimes} I_{\mathfrak{T}_{n}}, \Gamma_{1}^{A} \widehat{\otimes} I_{\mathfrak{T}_{n}}\right\}$ is a boundary triplet for $S_{n}^{*}$ such that

$$
S_{0 n}:=S^{*}\left\lceil\operatorname{ker}\left(\Gamma_{0}^{S_{n}}\right)=A_{0} \otimes I_{\mathfrak{T}_{n}}+I_{\mathfrak{H}} \otimes T_{n}, \quad n \in \mathbb{N} .\right.
$$

Let also $M^{S_{n}}(\cdot)$ be the corresponding Weyl function. It follows from (5.2) that

$$
\left(M^{S_{n}}\right)^{\prime}(z)=\int_{\Delta_{n}}\left(\left(M^{A}\right)^{\prime}(z-\lambda) \otimes I_{\mathfrak{T}_{n}}\right) \widehat{E}_{T}(d \lambda), \quad z \in \mathbb{C} \backslash \Delta_{n} .
$$

Since the function $\varphi(\cdot)=\sqrt{ }$ is continuous on $\mathbb{R}_{+}$, then in accordance with Proposition 3.3 (iii) the compositions $\left(\left(M^{A}\right)^{\prime}(a-\lambda)\right)^{1 / 2} \otimes I_{\mathfrak{T}_{n}}$ and $\left(\left(M^{A}\right)^{\prime}(a-\lambda)\right)^{-1 / 2} \otimes I_{\mathfrak{T}_{n}}$ are $\widehat{E}_{T_{n}}$-admissible. Therefore combining representation (5.22) with Proposition 3.3(iii) yields

$$
\begin{aligned}
R_{n} & :=\sqrt{\left(M^{S_{n}}\right)^{\prime}(a)}=\int_{\Delta_{n}}\left(\sqrt{\left(M^{A}\right)^{\prime}(a-\lambda)} \otimes I_{\mathfrak{T}_{n}}\right) \widehat{E}_{T}(d \lambda), \\
R_{n}^{-1} & =\frac{1}{\sqrt{\left(M^{S_{n}}\right)^{\prime}(a)}}=\int_{\Delta_{n}}\left(\frac{1}{\sqrt{\left(M^{A}\right)^{\prime}(a-\lambda)}} \otimes I_{\mathfrak{T}_{n}}\right) \widehat{E}_{T}(d \lambda),
\end{aligned}
$$

$a<0$. Similarly, using representations (5.2) and (5.23) and applying Proposition 3.2 yields

$$
\begin{aligned}
& R_{n}^{-1} M_{n}^{A}(a)=\frac{1}{\sqrt{\left(M^{S_{n}}\right)^{\prime}(a)}} M_{n}^{A}(a) \\
& \quad=\int_{\Delta_{n}}\left(\frac{1}{\sqrt{\left(M^{A}\right)^{\prime}(a-\lambda)}} M^{A}(a-\lambda) \otimes I_{\mathfrak{T}_{n}}\right) \widehat{E}_{T}(d \lambda), \quad a<0 .
\end{aligned}
$$

Setting $\mathcal{H}_{S_{n}}:=\mathcal{H}^{A} \otimes \mathfrak{T}_{n}$,

$$
\begin{aligned}
& \widetilde{\Gamma}_{0}^{S_{n}}=\sqrt{\left(M^{S_{n}}\right)^{\prime}(a)} \Gamma_{0}^{S_{n}} \quad \text { and } \\
& \left.\widetilde{\Gamma}_{1}^{S_{n}}=\frac{1}{\sqrt{\left(M^{S_{n}}\right)^{\prime}(a)}}\left(\Gamma_{1}^{S_{n}}-M^{S_{n}}(a)\right) \Gamma_{0}^{S_{n}}\right),
\end{aligned}
$$

we obtain an ordinary boundary triplet $\widetilde{\Pi}_{S_{n}}=\left\{\mathcal{H}_{S_{n}}, \widetilde{\Gamma}_{0}^{S_{n}}, \widetilde{\Gamma}_{1}^{S_{n}}\right\}$ for $S_{n}^{*}$. Inserting formulas (5.23) and (5.22) in (5.25) yields (5.20) with $\Delta_{n}$ in place of $\mathbb{R}_{+}$. Now applying Proposition 2.8 (see formula (2.16) ) one gets that the direct sum $\widetilde{\Pi}_{S}:=$ $\bigoplus_{n \in \mathbb{N}} \widetilde{\Pi}_{S_{n}}$ is an ordinary boundary triplet for $S^{*}$. In particular, for any $f \in$ 


$$
\begin{aligned}
\mathfrak{D}=\operatorname{dom} & \left(S^{*}\right) \cap \operatorname{dom}\left(A^{*} \otimes I_{\mathfrak{T}}\right) \\
\widetilde{\Gamma}_{0}^{S} f & :=\bigoplus_{n=1}^{\infty} \widetilde{\Gamma}_{0}^{S_{n}} f \\
& =\bigoplus_{n=1}^{\infty}\left(\int_{[n-1, n)}\left(\sqrt{\left(M^{A}\right)^{\prime}(a-\lambda)} \otimes I_{\mathfrak{T}_{n}}\right) \widehat{E}_{T_{n}}(d \lambda)\right) \cdot\left(\Gamma_{0}^{A} \widehat{\otimes} I_{\mathfrak{T}_{n}}\right) f \\
& =\left(\int_{\mathbb{R}_{+}}\left(\sqrt{\left(M^{A}\right)^{\prime}(a-\lambda)} \otimes I_{\mathfrak{T}}\right) \widehat{E}_{T_{n}}(d \lambda)\right) \cdot\left(\Gamma_{0}^{A} \widehat{\otimes} I_{\mathfrak{T}}\right) f
\end{aligned}
$$

which proves the first formula in (5.20). Note that convergence of the last integral for every $f \in \mathfrak{D}$ (cf. (5.3) ) is guaranteed by Lemma 5.2. Formula (5.20) for $\widetilde{\Gamma}_{1}^{S}$ is proved similarly.

(ii) It easily follows from (5.25) that the Weyl function $\widetilde{M}^{S_{n}}(\cdot)$ corresponding to the triplet $\widetilde{\Pi}_{S_{n}}$ is

$$
\begin{aligned}
\widetilde{M}^{S_{n}}(z) & =R_{n}^{-1}\left(M^{S_{n}}(z)-M^{S_{n}}(a)\right) R_{n}^{-1} \\
& =\frac{1}{\sqrt{\left(M^{S_{n}}\right)^{\prime}(a)}}\left(M^{S_{n}}(z)-M^{S_{n}}(a)\right) \frac{1}{\sqrt{\left(M^{S_{n}}\right)^{\prime}(a)}}
\end{aligned}
$$

Inserting formulas (5.23) and (5.2) into (5.27) and applying Proposition 3.2 we arrive at the following representation

$$
\begin{aligned}
\widetilde{M}^{S_{n}}(z)= & \int_{\Delta_{n}}\left(\frac{1}{\sqrt{\left(M^{A}\right)^{\prime}(a-\lambda)}} \times\right. \\
& \left.\times\left(M^{A}(z-\lambda)-M^{A}(a-\lambda)\right) \frac{1}{\sqrt{\left(M^{A}\right)^{\prime}(a-\lambda)}}\right) \widehat{E}_{T}(d \lambda),
\end{aligned}
$$

$z \in \mathbb{C}_{ \pm}$. Finally applying Proposition 2.8 and taking notation (5.8) into account we arrive at formula for the Weyl function $\widetilde{M}^{S}(\cdot)$ corresponding to $\widetilde{\Pi}_{S}$,

$$
\begin{aligned}
\widetilde{M}^{S}(z) f & =\bigoplus_{n \in \mathbb{N}} \widetilde{M}^{S_{n}}(z) f=\bigoplus_{n \in \mathbb{N}} \int_{\Delta_{n}}\left(L^{A}(z-\lambda, a-\lambda) \otimes I_{\mathfrak{T}}\right) \widehat{E}_{T}(d \lambda) f \\
& =\int_{\mathbb{R}_{+}}\left(L^{A}(z-\lambda, a-\lambda) \otimes I_{\mathfrak{T}}\right) \widehat{E}_{T}(d \lambda) f, \quad z \in \mathbb{C} \backslash \mathbb{R}_{+}
\end{aligned}
$$

exist for every $f \in \mathcal{H}^{A} \otimes \mathfrak{T}$ and any $z \in \mathbb{C} \backslash \mathbb{R}_{+}$. Note that Lemma 5.2 ensures convergence of the last integral for every $f \in \mathcal{H}^{A} \otimes \mathfrak{T}$. Comparison with (5.7) proves the second equality in (5.21). The first one is extracted by combining the first formula in (2.14) with (5.23) and applying Proposition 3.2

\subsection{Friedrichs and Krein extensions of $S:=A \otimes I_{\mathfrak{T}}+I_{\mathfrak{H}} \otimes T$.}

In this section we assume that both a symmetric operator $A \in \mathcal{C}(\mathfrak{H})$ and the operator $T=T^{*}$ are non-negative. Then the set $\operatorname{Ext}_{\mathrm{A}}[0, \infty)$ of non-negative 
self-adjoint extensions of $A$ is non-empty (see [2, 12, 38]). Moreover, according to the Krein result 45 the set $\operatorname{Ext}_{\mathrm{A}}[0, \infty)$ contains two extremal extensions: a maximal non-negative extension $\widehat{A}_{\mathrm{F}}$ (also called Friedrichs' or hard extension) and a minimal non-negative extension $\widehat{A}_{\mathrm{K}}$ (Krein's or soft extension). The latter are uniquely determined by the following inequalities

$$
\left(\widehat{A}_{F}+x\right)^{-1} \leq(\widetilde{A}+x)^{-1} \leq\left(\widehat{A}_{K}+x\right)^{-1}, \quad x \in(0, \infty), \quad \widetilde{A} \in \operatorname{Ext}_{\mathrm{A}}(0, \infty)
$$

(for detail we refer the reader to [2, 38]).

Recall the following statements.

Proposition 5.4 ([28]) Let $A \geq 0$ and let $\Pi=\left\{\mathcal{H}, \Gamma_{0}, \Gamma_{1}\right\}$ be a boundary triplet for $A^{*}$ such that $A_{0}\left(=A^{*} \uparrow \operatorname{ker} \Gamma_{0}\right) \geq 0$. Let $M(\cdot)$ be the corresponding Weyl function. Then $A_{0}=\widehat{A}_{\mathrm{F}}\left(A_{0}=\widehat{A}_{\mathrm{K}}\right)$ if and only if

$$
\lim _{x \downarrow-\infty}(M(x) f, f)=-\infty, \quad\left(\lim _{x \uparrow 0}(M(x) f, f)=+\infty\right), \quad f \in \mathcal{H} \backslash\{0\} .
$$

Next we describe the Friedrichs extension $\widehat{S}_{F}$ of $S$ by means of the extension $\widehat{A}_{F}$ of $A$. We start with the following simple algebraic lemma.

Lemma 5.5 Let $\left\{X_{k}\right\}_{1}^{n}$ be a sequence of positive definite operators in $\mathcal{H}, X_{k} \geq$ $d I_{\mathcal{H}}>0, d>0$, and let $E_{T}(\cdot)$ be a spectral measure of the operator $T=T^{*} \in$ $\mathcal{B}(\mathfrak{T})$. Then for any partition $\left\{\Delta_{k}\right\}_{1}^{n}$ of $[a, b](\sigma(T) \subset[a, b])$ one has

$$
X:=\sum_{k} X_{k} \otimes E_{T}\left(\Delta_{k}\right) \geq d I_{\mathcal{H} \otimes \mathfrak{T}}
$$

Proof. Since $X_{k} \geq d I_{\mathcal{H}}>0$, the operator $\left(X_{k}-d I_{\mathcal{H}}\right) \otimes E_{T}\left(\Delta_{k}\right)$ is non-negative. Hence

$$
\begin{aligned}
X & =\sum_{k} X_{k} \otimes E_{T}\left(\Delta_{k}\right) \geq d \sum_{k} I_{\mathcal{H}} \otimes E_{T}\left(\Delta_{k}\right) \\
& =d I_{\mathcal{H}} \otimes\left(\sum_{k} E_{T}\left(\Delta_{k}\right)\right)=d I_{\mathcal{H}} \otimes I_{\mathfrak{T}}=d I_{\mathcal{H} \otimes \mathfrak{T}}
\end{aligned}
$$

This inequality proves the result.

Proposition 5.6 Let $A$ be a non-negative symmetric operator in $\mathfrak{H}$, let $T=$ $T^{*} \geq 0$ and let $S:=A \otimes I_{\mathfrak{T}}+I_{\mathfrak{H}} \otimes T$. Then:

$$
\widehat{S}_{F}=\widehat{A}_{F} \otimes I_{\mathfrak{T}}+I_{\mathfrak{H}} \otimes T \quad \text { and } \quad \widehat{S}_{K}=\widehat{A}_{K} \otimes I_{\mathfrak{T}}+I_{\mathfrak{H}} \otimes T .
$$

Proof. (i) Assume for the beginning that $T$ is bounded, $T \in \mathcal{B}(\mathfrak{T})$. Let $\Pi_{A}=$ $\left\{\mathcal{H}^{A}, \Gamma_{0}^{A}, \Gamma_{1}^{A}\right\}$ be a boundary triplet for $A^{*}$ such that $A_{0}=\widehat{A}_{F}$. Then, by Theorem 5.1] $\Pi_{S}=\left\{\mathcal{H}^{S}, \Gamma_{0}^{S}, \Gamma_{1}^{S}\right\}:=\Pi_{A} \widehat{\otimes} I_{\mathfrak{T}}$ is a boundary triplet for $S^{*}$ satisfying $S_{0}:=S^{*} \uparrow \operatorname{ker}\left(\Gamma_{0}^{S}\right)=A_{0} \otimes I_{\mathfrak{T}}+I_{\mathfrak{H}} \otimes T$, and the corresponding Weyl function $M^{S}(\cdot)$ is given by (5.2). 
To prove the first relation in (5.30) it suffices to check condition (5.28) for $M^{S}(\cdot)$. Let $h:=\sum_{j=1}^{n} h_{j}^{\prime} \otimes h_{j}^{\prime \prime}$ where $h_{j}^{\prime} \in \mathcal{H}^{A}, h_{j}^{\prime \prime} \in \mathfrak{T}$, let $\mathcal{H}_{n}^{A}:=\operatorname{span}\left\{h_{j}^{\prime}: 1 \leq\right.$ $j \leq n\}$ and let $P_{n}$ be the orthogonal projection on $\mathcal{H}_{n}^{A}$ in $\mathcal{H}^{A}$.

Since $A_{0}=\widehat{A}_{F}$, the Weyl function $M^{A}(\cdot)$ satisfies condition (5.28). Setting $M_{n}^{A}(\cdot)=P_{n} M(\cdot)\left\lceil\mathcal{H}_{n}^{A}\right.$ we note that due to the compactness of the finitedimensional ball condition (5.28) is uniform on each $\mathcal{H}_{n}^{A}$. In other words, for each $N>0$ there exists $x_{N}<0$ such that

$$
-M_{n}^{A}(x) \geq N \quad \text { for } \quad x \leq x_{N} .
$$

Since $A_{0} \geq 0$, Theorem 5.1 ensures that the Weyl function $M^{A}(\cdot)$ being a holomorphic in $\mathbb{C} \backslash \mathbb{R}_{+}$admits the integral representation (5.2) for any $z=x<0$ and $\lambda>0$. Let $\pi=\left\{\Delta_{k}\right\}_{1}^{p}$ be a partition of $\Delta=[a, b]$, let $\lambda_{k} \in \Delta_{k}$, and let

$$
S_{p}(\pi)=\sum_{k=1}^{p} M^{A}\left(x_{N}-\lambda_{k}\right) \otimes E_{T}\left(\Delta_{k}\right)
$$

be an integral sum for the integral (5.2) with $x=x_{N}$. Setting $Y_{k}=M_{n}^{A}\left(x_{N}-\right.$ $\left.\lambda_{k}\right), k \in\{1, \ldots, p\}$, one gets

$$
\begin{array}{r}
\left(P_{n} \otimes I_{\mathfrak{T}}\right) S_{p}(\pi) h=\sum_{k=1}^{p} \sum_{j=1}^{n} P_{n} M^{A}\left(x_{N}-\lambda_{k}\right) h_{j}^{\prime} \otimes E_{T}\left(\Delta_{k}\right) h_{j}^{\prime \prime} \\
=\sum_{k=1}^{p} \sum_{j=1}^{n} Y_{k} h_{j}^{\prime} \otimes E_{T}\left(\Delta_{k}\right) h_{j}^{\prime \prime}=\sum_{k=1}^{p}\left(Y_{k} \otimes E_{T}\left(\Delta_{k}\right)\right) h .
\end{array}
$$

Combining this relation with (5.31) and noting that $h \in \mathcal{H}_{n}^{A} \otimes \mathfrak{T}$ and $x_{N}-\lambda_{k}<$ $x_{N}$ one gets from Lemma 5.5 that

$$
\left(S_{p}(\pi) h, h\right)=\left(\left(P_{n} \otimes I_{\mathfrak{T}}\right) S_{p}(\pi) h, h\right) \leq-N
$$

Passing here to the limit as the diameter $|\pi|$ of partition $\pi$ tends to zero and taking formula (5.2) for the Weyl function into account and setting $M_{n}^{S}(\cdot)=$ $\left(P_{n} \otimes I_{\mathfrak{T}}\right) M(\cdot)\left\lceil\mathcal{H}_{n}^{A} \otimes I_{\mathfrak{T}}\right.$, one derives

$$
\left(M^{S}(x) h, h\right)=\left(M_{n}^{S}(x) h, h\right) \leq-N \quad \text { for } \quad x \leq x_{N} .
$$

Since finite tensors $h=\sum_{j=1}^{n} h_{j}^{\prime} \otimes h_{j}^{\prime \prime}$ are dense in $\mathcal{H}^{A} \otimes \mathfrak{T}$, this inequality yields condition (5.28) for $M(\cdot)=M^{S}(\cdot)$ and arbitrary $h \in \mathcal{H}^{A} \otimes \mathfrak{T}$.

(ii) Let $T \in \mathcal{C}(\mathcal{H}) \backslash \mathcal{B}(\mathcal{H})$. Then $T$ admits a decomposition

$$
T=\bigoplus_{n \in \mathbb{N}} T_{n}
$$

where $T_{n}:=T E_{T}[n-1, n) \in \mathcal{B}\left(\mathcal{H}_{n}\right)$ and $\mathcal{H}_{n}:=E_{T}[n-1, n) \mathcal{H}$. Hence

$$
S=\bigoplus_{n \in \mathbb{N}} S_{n} \quad \text { where } \quad S_{n}:=A \otimes I_{\mathcal{H}_{n}}+I_{\mathfrak{H}} \otimes T_{n} .
$$


Clearly, $S_{n}$ is a non-negative symmetric operator in $\mathfrak{H} \otimes \mathcal{H}_{n}$. According to [50, Corollary 3.10]

$$
\widehat{S}_{F}=\bigoplus_{n \in \mathbb{N}} \widehat{S}_{n, F} \quad \text { and } \quad \widehat{S}_{K}=\bigoplus_{n \in \mathbb{N}} \widehat{S}_{n, K},
$$

where $\widehat{S}_{n, F}$ and $\widehat{S}_{n, K}$ denote the Friedrichs' and Krein's extensions of the symmetric non-negative operator $S_{n}$, respectively. Combining representations (5.35) with representations (5.30) with bounded $T_{n} \in \mathcal{B}\left(\mathcal{H}_{n}\right)$ in place of $T \in \mathcal{B}(\mathcal{H})$ proved at the previous step, implies

$$
\begin{aligned}
\widehat{S}_{F} & =\bigoplus_{n \in \mathbb{N}} \widehat{S}_{n, F}=\bigoplus_{n \in \mathbb{N}}\left(\widehat{A}_{F} \otimes I_{\mathcal{H}_{n}}+I_{\mathfrak{H}} \otimes T_{n}\right) \\
& =\widehat{A}_{F} \otimes I_{\mathcal{H}}+\bigoplus_{n \in \mathbb{N}}\left(I_{\mathfrak{H}} \otimes T_{n}\right)=\widehat{A}_{F} \otimes I_{\mathcal{H}}+I_{\mathfrak{H}} \otimes T .
\end{aligned}
$$

The representation for $S_{K}$ is proved similarly.

Next we are going to discuss semibounded extensions of the operator $S=A \otimes$ $I_{\mathfrak{T}}+I_{\mathfrak{H}} \otimes T$. It is known that under the conditions of Proposition 5.4 the following implication holds: $\widetilde{A}=\widetilde{A}^{*}=A_{\Theta}$ is semi-bounded below then $\Theta$ is semi-bounded below. The equivalence does not hold in general.

Definition 5.7 Let $A \geq 0$ be a non-negative symmetric operator in $\mathfrak{H}$ and let $\Pi=\left\{\mathcal{H}, \Gamma_{0}, \Gamma_{1}\right\}$ be a boundary triplet for $A^{*}$ such that $A_{0}=\widetilde{A}_{F}$. We say that $A$ satisfies LSB-property (abbreviation of lower semi-boundedness) if the following equivalence holds:

$$
A_{\Theta}=A_{\Theta}^{*} \text { is lower semi-bounded } \Longleftrightarrow \Theta=\Theta^{*} \text { is lower semi-bounded. }
$$

To describe the operators with LSB-property we introduce the following definition.

Definition $5.8([28])$ It is said that $M(\cdot)$ uniformly tends to $-\infty$ (in symbols $M(\cdot) \rightrightarrows-\infty)$ if for any $N>0$ there exists $x_{N}$ such that

$$
(M(x) h, h) \leq-N \cdot\|h\|^{2} \quad \text { for } x \leq x_{N}, \quad h \in \mathcal{H} .
$$

Clearly, (5.36) implies (5.28) but not vice versa.

Proposition $5.9([28])$ Let $A \geq 0$ and let $\Pi=\left\{\mathcal{H}, \Gamma_{0}, \Gamma_{1}\right\}$ be a boundary triplet for $A^{*}$ such that $A_{0}=\widetilde{A}_{F}$. Then the following statements are equivalent:

(i) A satisfies LSB property;

(ii) $M(x) \rightrightarrows-\infty$ as $x \rightarrow-\infty$.

Proposition 5.10 Let $A$ be a non-negative symmetric operator in $\mathfrak{H}$ and let $\Pi_{A}=\left\{\mathcal{H}^{A}, \Gamma_{0}^{A}, \Gamma_{1}^{A}\right\}$ be a boundary triplet for $A^{*}$ such that $A_{0}:=A^{*} \mid$ $\operatorname{ker}\left(\Gamma_{0}^{A}\right)=\widehat{A}_{F}$. Let also $T=T^{*} \in \mathcal{B}(\mathfrak{T}), T \geq 0$ and let $S=A \otimes I_{\mathfrak{T}}+I_{\mathfrak{H}} \otimes T$. If $A$ satisfies the LSB-property, then the operator $S$ also satisfies the LSB-property. 
Proof. Consider a boundary triplet $\widetilde{\Pi}_{S}=\left\{\widetilde{\mathcal{H}}^{S}, \widetilde{\Gamma}_{0}^{S}, \widetilde{\Gamma}_{1}^{S}\right\}$ for $S^{*}$ given by (5.20). By Theorem 5.1(i),

$$
S_{0}=S^{*} \uparrow \operatorname{ker}\left(\Gamma_{0}^{S}\right)=\widehat{S}_{F}=\widehat{A}_{F} \otimes I_{\mathfrak{T}}+I_{\mathfrak{H}} \otimes T .
$$

Let also $M^{A}(\cdot)$ and $\gamma^{A}(\cdot)$ be the Weyl function and $\gamma$-field, respectively, corresponding to the triplet $\Pi_{A}$. Since $A$ satisfies the LSB-property and $A_{0}=\widehat{A}_{F}$, Theorem [5.9] ensures that the Weyl function $M^{A}(\cdot)$ tends to $-\infty$ uniformly, i.e. $M^{A}(x) \rightrightarrows-\infty$ as $x \rightarrow-\infty$. In other words, for each $N>0$ there exists $x_{N}<0$ such that $-M^{A}(x) \geq N$ for $x \leq x_{N}$.

By Theorem [5.1(i) the Weyl function $M^{S}(\cdot)$ corresponding to $\Pi_{S}$ is given by (5.2). Let $\pi=\left\{\Delta_{k}\right\}_{1}^{p}$ be a partition of $\Delta=[a, b]$ and let $\lambda_{k} \in \Delta_{k}$. Then applying Lemma 5.5 to the integral sum (5.32) we get

$$
-S_{p}(\pi)=-\sum_{k=1}^{p} M^{A}\left(x_{N}-\lambda_{k}\right) \otimes E_{T}\left(\Delta_{k}\right) \geq N .
$$

Passing here to the limit as $|\pi| \rightarrow 0$ one obtains

$$
-M^{S}(x)=\int_{\Delta} \widehat{E}_{T}(d \lambda)\left(M^{A}(z-\lambda) \otimes I_{\mathfrak{T}}\right) \geq N \quad \text { for } \quad x \leq x_{N} .
$$

The latter amounts to saying that $M^{S}(x) \rightrightarrows-\infty$ as $x \rightarrow-\infty$. By Theorem 5.9 this property implies (in fact is equivalent to) the LSB-property of $S$.

\section{Examples}

In what follows the operator $T$ is arbitrary (not necessarily bounded) self-adjoint operator acting on a separable Hilbert space $\mathfrak{T}$.

\subsection{Schrödinger operators and bosons in 1D}

\subsubsection{Schrödinger operators on half-lines}

Let $v_{r} \in \mathbb{R}, b \in \mathbb{R}$, and let $H_{r}=-\frac{d^{2}}{d x^{2}}+v_{r}$ denote a minimal operator in $\mathfrak{H}_{r}:=$ $L^{2}\left(\Delta_{r}\right), \Delta_{r}=(b, \infty)$. Clearly, $\operatorname{dom}\left(H_{r}\right)=W_{0}^{2,2}\left(\Delta_{r}\right):=\left\{f \in W_{2}^{2}((b, \infty)):\right.$ $\left.f(b)=f^{\prime}(b)=0\right\}$ and $H_{r}$ is a closed densely defined symmetric operator with $n_{ \pm}\left(H_{r}\right)=1$. The adjoint operator is given by the same expression $H_{r}^{*}=$ $-\frac{d^{2}}{d x^{2}}+v_{r}$ on the domain $\operatorname{dom}\left(H_{r}^{*}\right)=W^{2,2}\left(\Delta_{r}\right)$. One easily checks that a triplet $\Pi_{H_{r}}=\left\{\mathcal{H}^{H_{r}}, \Gamma_{0}^{H_{r}}, \Gamma_{1}^{H_{r}}\right\}$ with

$$
\mathcal{H}^{H_{r}}:=\mathbb{C}, \quad \Gamma_{0}^{H_{r}} f=f(b), \quad \text { and } \quad \Gamma_{1}^{H_{r}} f=f^{\prime}(b), \quad f \in \operatorname{dom}\left(H_{r}{ }^{*}\right),
$$

is a boundary triplet for $H_{r}^{*}$. The corresponding $\gamma$-field $\gamma^{H_{r}}(\cdot)$ and Weyl function $M^{H_{r}}(\cdot)$ are given by

$$
\left(\gamma^{H_{r}}(z) \xi\right)(x)=e^{i \sqrt{z-v_{r}}(x-b)} \xi, \quad \xi \in \mathbb{C}, \quad x \in \Delta_{r}, \quad z \in \mathbb{C}_{ \pm},
$$


and

$$
M^{H_{r}}(z)=m^{H_{r}}(z)=i \sqrt{z-v_{r}}, \quad z \in \mathbb{C}_{ \pm},
$$

respectively. The function $\sqrt{\cdot}$ is defined on $\mathbb{C}$ with the cut along the positive semi-axis $\mathbb{R}_{+}$. Its branch is fixed by the condition $\sqrt{1}=1$. Clearly, the Weyl function $M^{H_{r}}(\cdot)$ is a scalar function.

Let us consider the closed densely defined symmetric operator

$$
S_{r}=\overline{H_{r} \otimes I_{\mathfrak{T}}+I_{\mathfrak{H}_{r}} \otimes T}
$$

on the Hilbert space $\mathfrak{K}_{r}:=\mathfrak{H}_{r} \otimes \mathfrak{T}=L_{2}\left(\Delta_{r}, \mathfrak{T}\right)$. In the following we use the notation $\vec{f}(x), x \in \Delta_{r}$ for elements of $\mathfrak{K}_{r}=L_{2}\left(\Delta_{r}, \mathfrak{T}\right)$. In accordance with Theorem 4.8 (iii) there is a boundary triplet $\Pi_{S_{r}}=\left\{\mathcal{H}^{S_{r}}, \Gamma_{0}^{S_{r}}, \Gamma_{1}^{S_{r}}\right\}$ for $S_{r}{ }^{*}$ such that $\mathcal{H}^{S_{r}}=\mathcal{H}^{H_{r}} \otimes \mathfrak{T}=\mathfrak{T}$,

$$
\begin{aligned}
& \Gamma_{0}^{S_{r}} \vec{f}=\sqrt{\operatorname{Im}\left(m^{H_{r}}(i-T)\right)} \vec{f}(b), \\
& \Gamma_{1}^{S_{r}} \vec{f}=\frac{1}{\sqrt{\operatorname{Im}\left(m^{H_{r}}(i-T)\right)}}\left(\vec{f}^{\prime}(b)-\operatorname{Re}\left(m^{H_{r}}(i-T)\right) \vec{f}(b)\right),
\end{aligned}
$$

$\vec{f} \in \operatorname{dom}\left(H_{r}{ }^{*} \otimes I_{\mathfrak{T}}\right) \cap \operatorname{dom}\left(I_{\mathfrak{H}_{r}} \otimes T\right)=W^{2,2}\left(\Delta_{r}, \mathfrak{T}\right) \cap \operatorname{dom}\left(I_{\mathfrak{H}_{r}} \otimes T\right) \subseteq \operatorname{dom}\left(S_{r}^{*}\right)$. The corresponding $\gamma$-field $\gamma^{S_{r}}(\cdot): \mathfrak{T} \longrightarrow \mathfrak{K}_{r}$ and Weyl function $M^{S_{r}}(\cdot): \mathfrak{T} \longrightarrow \mathfrak{T}$ are given by

$$
\left(\gamma^{S_{r}} \xi\right)(x)=e^{i \sqrt{z-v_{r}-T}(x-b)} \frac{1}{\sqrt{\operatorname{Im}\left(m^{H_{r}}(i-T)\right)}} \xi, \quad \xi \in \mathfrak{T}, \quad x \in \Delta_{r},
$$

and

$$
M^{S_{r}}(z)=\frac{m^{H_{r}}(z-T)-\operatorname{Re}\left(m^{H_{r}}(i-T)\right)}{\operatorname{Im}\left(m^{H_{r}}(i-T)\right)}, \quad z \in \mathbb{C}_{ \pm} .
$$

Of course, the considerations are similar for the interval $\Delta_{l}=(-\infty, a)$, $a \in \mathbb{R}$. Let $H_{l}=-\frac{d^{2}}{d x^{2}}+v_{l}, v_{l} \in \mathbb{R}$, with domain $\operatorname{dom}\left(H_{l}\right):=W_{0}^{2,2}\left(\Delta_{l}\right)$ defined on $\mathfrak{H}_{l}:=L^{2}\left(\Delta_{l}, \mathfrak{T}\right)$. One checks that $\Pi_{H_{l}}=\left\{\mathcal{H}^{H_{l}}, \Gamma_{0}^{H_{l}}, \Gamma_{1}^{H_{l}}\right\}$,

$$
\mathcal{H}^{H_{l}}:=\mathbb{C}, \quad \Gamma_{0}^{H_{l}} f=f(a), \quad \text { and } \quad \Gamma_{1}^{H_{l}} f=-f^{\prime}(a), \quad f \in \operatorname{dom}\left(H_{l}{ }^{*}\right),
$$

is a boundary triplet for $H_{l}^{*}$. The $\gamma$-field and Weyl function are given by

$$
\left(\gamma^{H_{l}}(z) \xi\right)(x)=e^{i \sqrt{z-v_{l}}(a-x)} \xi, \quad \xi \in \mathbb{C}, \quad x \in \Delta_{l}, \quad z \in \mathbb{C}_{ \pm},
$$

and

$$
M^{H_{l}}(z)=m^{H_{l}}(z)=i \sqrt{z-v_{l}}, \quad z \in \mathbb{C}_{ \pm} .
$$

Let us consider the closed densely defined symmetric operator $S_{l}=$ $\overline{H_{l} \otimes I_{\mathfrak{T}}+I_{\mathfrak{H}_{l}} \otimes T}$ acting in $\mathfrak{K}_{l}:=\mathfrak{H}_{l} \otimes \mathfrak{T}=L^{2}\left(\Delta_{l}, \mathfrak{T}\right)$. As above one finds

$$
\begin{aligned}
& \Gamma_{0}^{S_{l}} \vec{f}=\sqrt{\operatorname{Im}\left(m^{H_{l}}(i-T)\right)} \vec{f}(a), \\
& \left.\Gamma_{1}^{S_{l}} f=\frac{1}{\sqrt{\operatorname{Im}\left(m^{H_{l}}(i-T)\right)}}\left(-\vec{f}^{\prime}(a)-\operatorname{Re}\left(m^{H_{l}}(i-T)\right) \vec{f}(a)\right)\right)
\end{aligned}
$$


$\vec{f} \in \operatorname{dom}\left(H_{l}{ }^{*} \otimes I_{\mathfrak{T}}\right) \cap \operatorname{dom}\left(I_{\mathfrak{H}_{l}} \otimes T\right)=W^{2,2}\left(\Delta_{l}, \mathfrak{T}\right) \cap \operatorname{dom}\left(I_{\mathfrak{H}_{l}} \otimes T\right) \subseteq \operatorname{dom}\left(S_{l}^{*}\right)$ as well as

$$
\left(\gamma^{S_{l}} \xi\right)(x)=e^{i \sqrt{z-v_{l}-T}(a-x)} \frac{1}{\sqrt{\operatorname{Im}\left(m^{H_{l}}(i-T)\right)}} \xi, \quad \xi \in \mathfrak{T}, \quad x \in \Delta_{r},
$$

and

$$
M^{S_{l}}(z)=\frac{m^{H_{l}}(z-T)-\operatorname{Re}\left(m^{H_{l}}(i-T)\right)}{\operatorname{Im}\left(m^{H_{l}}(i-T)\right)}, \quad z \in \mathbb{C}_{ \pm} .
$$

\subsubsection{Schrödinger operators on bounded intervals}

Let $\Delta_{c}=(a, b)$ and $v_{c} \in \mathbb{R}$. Consider a minimal Sturm-Liouville operator $H_{c}$ in $\mathfrak{H}_{c}=L^{2}\left(\Delta_{c}\right)$ given by

$$
\begin{gathered}
\left(H_{c} f\right)(x)=-\frac{d^{2}}{d x^{2}} f(x)+v_{c} f(x), \quad x \in \Delta_{c} \\
f \in \operatorname{dom}\left(H_{c}\right)=\left\{\begin{array}{l}
f(a)=f(b)=0 \\
f \in W^{2,2}\left(\Delta_{c}\right): \\
f^{\prime}(a)=f^{\prime}(b)=0
\end{array}\right\} .
\end{gathered}
$$

Clearly, $H_{c}$ is a closed symmetric operator with the deficiency indices $n_{ \pm}(A)=$ 2. Its adjoint $H_{c}^{*}$ is given by

$$
\left(H_{c}^{*} f\right)(x)=-\frac{d^{2}}{d x^{2}} f(x)+v_{c} f(x), \quad f \in \operatorname{dom}\left(H_{c}^{*}\right)=W^{2,2}\left(\Delta_{c}\right) .
$$

Consider the extension (Dirichlet realization) $H_{c}^{D}$ of the minimal operator $H_{c}$ defined by

$$
H_{c}^{D}=-\frac{d^{2}}{d x^{2}}+v_{c}, \quad \operatorname{dom}\left(H_{c}^{D}\right)=\left\{f \in W^{2,2}\left(\Delta_{c}\right): f(a)=f(b)=0\right\} .
$$

The Neumann extension (realization) $H_{N}$ is fixed by

$$
H_{c}^{N}=-\frac{d^{2}}{d x^{2}}+v_{c}, \quad \operatorname{dom}\left(H_{c}^{N}\right)=\left\{f \in W^{2,2}\left(\Delta_{c}\right): f^{\prime}(a)=f^{\prime}(b)=0\right\} .
$$

One easily checks that the triplet $\Pi_{H_{c}}:=\left\{\mathcal{H}^{H_{c}}, \Gamma_{0}^{H_{c}}, \Gamma_{1}^{H_{c}}\right\}$ with

$$
\mathcal{H}^{H_{c}}:=\mathbb{C}^{2}, \quad \Gamma_{0}^{H_{c}} f=\frac{1}{\sqrt{2}}\left(\begin{array}{c}
f(a)+f(b) \\
f(a)-f(b)
\end{array}\right), \quad \Gamma_{1}^{H_{c}} f=\frac{1}{\sqrt{2}}\left(\begin{array}{c}
f^{\prime}(a)-f^{\prime}(b) \\
f^{\prime}(a)+f^{\prime}(b)
\end{array}\right),
$$

$f \in \operatorname{dom}\left(H_{c}^{*}\right)$, is a boundary triplet for $H_{c}^{*}$. Clearly, $H_{c}^{D}=H_{c}^{*}\left\lceil\operatorname{ker}\left(\Gamma_{0}^{H_{c}}\right)\right.$ and $H_{c}^{N}=H_{c}^{*}\left\lceil\operatorname{ker}\left(\Gamma_{1}^{H_{c}}\right)\right.$. The corresponding $\gamma$-field $\gamma^{H_{c}}(\cdot)$ and Weyl function $M^{H_{c}}(\cdot)$ are given by

$$
\left(\gamma^{H_{c}}(z) \xi\right)(x)=\frac{1}{\sqrt{2}}\left(\frac{\cos \left(\sqrt{z-v_{c}}(x-\nu)\right)}{\cos \left(\sqrt{z-v_{c}} d\right)},-\frac{\sin \left(\sqrt{z-v_{c}}(x-\nu)\right)}{\sin \left(\sqrt{z-v_{c}} d\right)}\right) \cdot\left(\begin{array}{l}
\xi_{1} \\
\xi_{2}
\end{array}\right),
$$


$z \in \mathbb{C}_{ \pm}, x \in(a, b), \nu:=\frac{a+b}{2}, d:=\frac{b-a}{2}$, and

$$
M^{H_{c}}(z)=\left(\begin{array}{cc}
m_{1}^{H_{c}}(z) & 0 \\
0 & m_{2}^{H_{c}}(z)
\end{array}\right), \quad z \in \mathbb{C}_{ \pm},
$$

where

$$
\begin{aligned}
& m_{1}^{H_{c}}(z):=\sqrt{z-v_{c}} \tan \left(\sqrt{z-v_{c}} d\right), \\
& m_{2}^{H_{c}}(z):=-\sqrt{z-v_{c}} \cot \left(\sqrt{z-v_{c}} d\right),
\end{aligned} \quad z \in \mathbb{C}_{ \pm} .
$$

Notice that the Weyl function $M^{H_{c}}(\cdot)$ is of quasi scalar type.

We consider the closed densely defined symmetric operator

$$
S_{c}:=\overline{H_{c} \otimes I_{\mathfrak{T}}+I_{\mathfrak{H}_{H}} \otimes T} .
$$

defined on $\mathfrak{K}_{c}:=\mathfrak{H}_{c} \otimes \mathfrak{T}=L^{2}\left(\Delta_{c}, \mathfrak{T}\right)$. Elements of $L^{2}\left(\Delta_{c}, \mathfrak{T}\right)$ are denoted by $\vec{f}(x), x \in \Delta_{c}$. Obviously, the self-adjoint operators $S_{c}^{D}:=\overline{H_{c}^{D} \otimes I_{\mathfrak{T}}+I_{\mathfrak{H}_{H}} \otimes T}$ and $S_{c}^{N}:=\overline{H_{c}^{N} \otimes I_{\mathfrak{T}}+I_{\mathfrak{H}_{H}} \otimes T}$ are self-adjoint extensions of $S_{c}$.

Let us introduce the subspaces $\mathcal{H}_{1}^{H_{c}}:=\mathbb{C}$ and $\mathcal{H}_{2}^{H_{c}}:=\mathbb{C}$. Notice that $\mathcal{H}^{H_{c}}=\left(\mathcal{H}_{1}^{H_{c}} \oplus_{\mathcal{H}_{2}}^{H_{c}}\right)^{t}$. It follows from (4.32) that there is a boundary triplet $\Pi_{S_{c}}=\left\{\mathcal{H}^{S_{c}}, \Gamma_{0}^{S_{c}}, \Gamma_{1}^{S_{c}}\right\}$ for $S_{c}^{*}$ such that

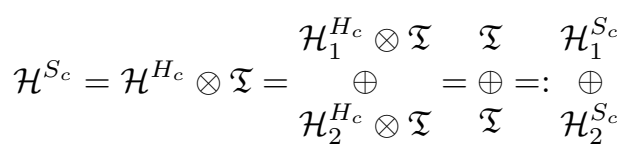

and

$$
\begin{aligned}
& \Gamma_{0}^{S_{c}} \vec{f}=\frac{1}{\sqrt{2}}\left(\begin{array}{c}
\sqrt{\operatorname{Im}\left(m_{1}^{H_{c}}(i-T)\right.}(\vec{f}(a)+\vec{f}(b) \\
\sqrt{\operatorname{Im}\left(m_{2}^{H_{c}}(i-T)\right)}(\vec{f}(a)-\vec{f}(b))
\end{array}\right) \\
& \Gamma_{1}^{S_{c}}(z) \vec{f}= \\
& \frac{1}{\sqrt{2}}\left(\begin{array}{c}
\frac{1}{\sqrt{\operatorname{Im}\left(m_{1}^{H_{c}}(i-T)\right.}}\left(\vec{f}^{\prime}(a)-\vec{f}^{\prime}(b)-\operatorname{Re}\left(m_{1}^{H_{c}}(i-T)\right)(\vec{f}(a)+\vec{f}(b))\right. \\
\left.\frac{1}{\sqrt{\operatorname{Im}\left(m_{2}^{H_{c}}(i-T)\right)}}\left(\vec{f}^{\prime}(a)+\vec{f}^{\prime}(b)\right)-\operatorname{Re}\left(m_{2}^{H_{c}}(i-T)\right)(\vec{f}(a)-\vec{f}(b))\right)
\end{array}\right)
\end{aligned}
$$

$\vec{f} \in \operatorname{dom}\left(H_{c}^{*} \otimes I_{\mathfrak{T}}\right) \cap \operatorname{dom}\left(I_{\mathfrak{H}_{c}} \otimes T\right)=W^{2,2}\left(\Delta_{c}, \mathfrak{T}\right) \cap \operatorname{dom}\left(I_{\mathfrak{H}_{c}} \otimes T\right) \subseteq \operatorname{dom}\left(S_{c}^{*}\right)$.

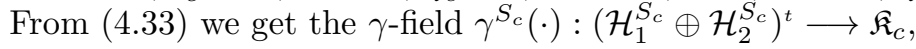

$$
\begin{aligned}
\left(\gamma^{S_{c}}(z) \vec{\xi}\right)(x)= & \frac{\cos \left(\sqrt{z-T-v_{c}}(x-\nu)\right)}{\sqrt{2} \cos \left(\sqrt{z-T-v_{c}} d\right) \sqrt{\operatorname{Im}\left(m_{1}^{H_{c}}(i-T)\right)}} \vec{\xi}_{1} \\
& -\frac{\sin \left(\sqrt{z-T-v_{c}}(x-\nu)\right)}{\sqrt{2} \sin \left(\sqrt{z-T-v_{c}} d\right) \sqrt{\operatorname{Im}\left(m_{2}^{H_{c}}(i-T)\right)}} \vec{\xi}_{2},
\end{aligned}
$$




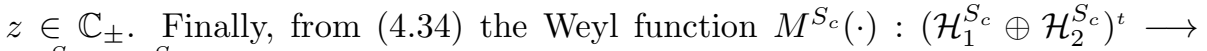
$\left(\mathcal{H}_{1}^{S_{c}} \oplus \mathcal{H}_{2}^{S_{c}}\right)^{t}$ is computed by

$$
M^{S_{c}}(z)=\left(\begin{array}{cc}
\frac{m_{1}^{H_{c}}(z-T)-\operatorname{Re}\left(m_{1}^{H_{c}}(i-T)\right)}{\operatorname{Im}\left(m_{1}^{H_{c}}(i-T)\right)} & 0 \\
0 & \frac{m_{2}^{H_{c}}(z-T)-\operatorname{Re}\left(m_{2}^{H_{c}}(i-T)\right)}{\operatorname{Im}\left(m_{2}^{H_{c}}(i-T)\right)}
\end{array}\right), \quad z \in \mathbb{C}_{ \pm} .
$$

Remark 6.1 Sturm-Liouville operators $S_{c}$ with operator-valued potential $T=$ $T^{*} \in \mathcal{C}(\mathfrak{T})$ have first been treated on a finite interval in the pioneering paper by M.L. Gorbachuk 31. Clearly, the corresponding minimal operator $S_{c}$ admits representation (6.8). In particular, a boundary triplet for $S_{c}^{*}$ was first constructed in 31] (see also 32]). A construction of a boundary triplet for $S_{r}^{*}$ in the case of semi-axis has first been proposed in [28, Section 9]. However, our construction (6.3) of the boundary triplet for $S_{r}^{*}$ is borrowed from [50] where a representation of $S$ as a direct sum $S=\bigoplus_{j} S_{j}$ with bounded $T_{j}$ in place of unbounded $T$ was first proposed and the regularization procedure for direct sums was invented and applied to the operator $S_{r}$.

After appearance of the work 31 the spectral theory of self-adjoint and dissipative extensions of $S_{c}$ in $L^{2}\left(\Delta_{c}, \mathfrak{T}\right)$ has intensively been investigated. The results are summarized in [32, Chapter 4] where one finds, in particular, criteria for discreteness of the spectra, asymptotic formulas for the eigenvalues, resolvent comparability results, etc. Spectral properties of self-adjoint extensions of $S_{r}$ was investigated in [50], see also [49. In particular, a criterion for all selfadjoint extensions of $S_{r}$ to have absolutely continuous non-negative spectra was also obtained there.

\subsection{Dirac operators and bosons in 1D}

In the following we consider the Dirac operator instead of the Schrödinger operator, cf. [20], [13].

\subsubsection{Dirac operators on half-lines}

In the Hilbert space $\mathfrak{D}_{r}=L^{2}\left(\Delta_{r}, \mathbb{C}^{2}\right), \Delta_{r}=(b, \infty)$, let us consider the Dirac operator

$$
\begin{gathered}
D_{r} f:=\left(-i c \frac{d}{d x} \otimes \sigma_{1}\right) f+\left(\frac{c^{2}}{2} \otimes \sigma_{3}\right) f \\
f \in \operatorname{dom}\left(D_{r}\right):=W_{0}^{1,2}\left(\Delta_{r}, \mathbb{C}^{2}\right):=\left\{f \in W^{1,2}\left(\Delta_{r}, \mathbb{C}^{2}\right): f(b)=0\right\} .
\end{gathered}
$$

Here

$$
\sigma_{1}:=\left(\begin{array}{ll}
0 & 1 \\
1 & 0
\end{array}\right) \quad \text { and } \quad \sigma_{3}:=\left(\begin{array}{cc}
1 & 0 \\
0 & -1
\end{array}\right)
$$

Notice that

$$
D_{r}^{*} f=\left(-i c \frac{d}{d x} \otimes \sigma_{1}\right) f+\left(\frac{c^{2}}{2} \otimes \sigma_{3}\right) f, \quad f \in \operatorname{dom}\left(D_{r}^{*}\right)=W^{1,2}\left(\Delta_{r}, \mathbb{C}^{2}\right) .
$$


One easily checks that $n_{ \pm}\left(D_{r}\right)=1$. From Lemma 3.3 of [20] we get that $\Pi_{D_{r}}=\left\{\mathcal{H}^{D_{r}}, \Gamma_{0}^{D_{r}}, \Gamma_{1}^{D_{r}}\right\}$

$$
\mathcal{H}^{D_{r}}:=\mathbb{C}, \quad \Gamma_{0}^{D_{r}} f:=f_{1}(b), \quad \Gamma_{1}^{D_{r}} f:=i c f_{2}(b)
$$

is a boundary triplet for $D_{r}^{*}$. The $\gamma$-field $\gamma^{D_{r}}(\cdot)$ and Weyl function are given by

$$
\left(\gamma^{D_{r}}(z) \xi\right)(x)=\left(\begin{array}{c}
e^{i k(z)(x-b)} \xi \\
k_{1}(z) e^{i k(z)(x-b)} \xi
\end{array}\right), \quad x \in \Delta_{r}, \quad z \in \mathbb{C}_{ \pm}, \quad \xi \in \mathcal{H}^{D_{r}} .
$$

and

$$
M^{D_{r}}(z)=m^{D_{r}}(z) I_{\mathcal{H}^{D_{r}}}, \quad m_{r}(z):=i c k_{1}(z), \quad z \in \mathbb{C}_{ \pm} .
$$

Here

$$
k(z):=\frac{1}{c} \sqrt{z^{2}-\frac{c^{4}}{4}}, \quad z \in \mathbb{C},
$$

where the branch of the multifunction $k(\cdot)$ is fixed by the condition $k(x)>0$ for $x>\frac{c^{2}}{2}$. Notice that $k(\cdot)$ is holomorphic on $\mathbb{C} \backslash\left\{\left(-\infty,-\frac{c^{2}}{2}\right] \cup\left[\frac{c^{2}}{2}, \infty\right)\right\}$. Further, let

$$
k_{1}(z):=\frac{c k(z)}{z+\frac{c^{2}}{2}}, \quad z \in \mathbb{C}
$$

Clearly, $k_{1}(\cdot)$ is also holomorphic on $\mathbb{C} \backslash\left\{\left(-\infty,-\frac{c^{2}}{2}\right] \cup\left[\frac{c^{2}}{2}, \infty\right)\right\}$ and admits the representation

$$
k_{1}(z)=\sqrt{\frac{z-\frac{c^{2}}{2}}{z+\frac{c^{2}}{2}}}, \quad z \in \mathbb{C},
$$

where the branch of $\sqrt{\frac{z-\frac{c^{2}}{2}}{z+\frac{c^{2}}{2}}}$ is fixed by the condition $\sqrt{\frac{x-\frac{c^{2}}{2}}{x+\frac{c^{2}}{2}}}>0$ for $x>\frac{c^{2}}{2}$.

Let us consider the closed densely defined symmetric operator

$$
S_{r}=\overline{D_{r} \otimes I_{\mathfrak{T}}+I_{\mathfrak{D}_{r}} \otimes T},
$$

which is defined on

$$
\mathfrak{K}_{r}:=\mathfrak{D}_{r} \otimes \mathfrak{T}=L^{2}\left(\Delta_{r},(\mathfrak{T} \oplus \mathfrak{T})^{t}\right) .
$$

In the following we denote elements of $\mathfrak{K}_{r}$ by $\vec{f}=\left(\vec{f}_{1}, \vec{f}_{2}\right)^{t}$. Since $D_{r}$ is not semi-bounded from below the sum $\overline{D_{r} \otimes I_{\mathfrak{T}}+I_{\mathfrak{D}_{r}} \otimes T}$ is also not semibounded from below. Nevertheless, by Theorem 4.8(iii) a boundary triplet $\Pi_{S_{r}}=\left\{\mathcal{H}^{S_{r}}, \Gamma_{0}^{S_{r}}, \Gamma_{1}^{S_{r}}\right\}$ for $S_{r}^{*}$ can be chosen in the form

$$
\mathcal{H}^{S_{r}}=\mathcal{H}^{D_{r}} \otimes \mathfrak{T}=\mathfrak{T}
$$

and

$$
\begin{aligned}
& \Gamma_{0}^{S_{r}} \vec{f}=\sqrt{\operatorname{Im}\left(m^{D_{r}}(i-T)\right)} \vec{f}_{1}(b), \\
& \Gamma_{1}^{S_{r}} \vec{f}=\frac{1}{\sqrt{\operatorname{Im}\left(m^{D_{r}}(i-T)\right)}}\left(i c \vec{f}_{2}(b)-\operatorname{Re}\left(m_{1}^{D_{r}}(i-T)\right) \vec{f}_{1}(b)\right),
\end{aligned}
$$


$\vec{f} \in \operatorname{dom}\left(D_{r}^{*} \otimes I_{\mathfrak{T}}\right) \cap \operatorname{dom}\left(I_{\mathfrak{D}_{r}} \otimes T\right)=W^{2,2}\left(\Delta_{r},(\mathfrak{T} \oplus \mathfrak{T})^{t}\right) \cap \operatorname{dom}\left(I_{\mathfrak{D}_{r}} \otimes T\right) \subseteq$ $\operatorname{dom}\left(S_{r}^{*}\right)$. The $\gamma$-field $\gamma^{L_{r}}(\cdot): \mathfrak{T} \longrightarrow \mathfrak{K}_{r}$ and Weyl function $M^{S_{r}}(\cdot): \mathfrak{T} \longrightarrow \mathfrak{T}$ are given by

$$
\left(\gamma^{S_{r}}(z) \xi\right)(x)=\left(\begin{array}{c}
e^{i k(z-T)(x-b)} \frac{1}{\operatorname{Im}\left(m^{\left.D_{r}(i-T)\right)}\right.} \xi \\
k_{1}(z-T) e^{i k(z-T)(x-b)} \frac{1}{\operatorname{Im}\left(m^{D_{r}}(i-T)\right)} \xi
\end{array}\right), \quad \xi \in \mathfrak{T},
$$

$x \in \Delta_{r}, z \in \mathbb{C}_{ \pm}$and

$$
M^{S_{r}}(z)=\frac{m^{D_{r}}(z-T)-\operatorname{Re}\left(m^{D_{r}}(i-T)\right)}{\operatorname{Im}\left(m^{D_{r}}(i-T)\right)}, \quad z \in \mathbb{C}_{ \pm} .
$$

The Dirac operator on the half-axis $(-\infty, a)$ is treated similarly.

\subsubsection{Dirac operators on bounded intervals}

Let us consider the closed densely defined symmetric operator

$$
\begin{gathered}
D_{c} f:=\left(-i c \frac{d}{d x} \otimes \sigma_{1}\right) f+\left(\frac{c^{2}}{2} \otimes \sigma_{3}\right) f, \\
f \in \operatorname{dom}\left(D_{c}\right):=W_{0}^{1,2}\left(\Delta_{c}, \mathbb{C}^{2}\right):=\left\{f \in W^{1,2}\left(\Delta_{c}, \mathbb{C}^{2}\right): f(a)=f(b)=0\right\},
\end{gathered}
$$

where $\Delta_{c}=(a, b)$, acting in the Hilbert space $\mathfrak{D}_{c}:=L^{2}\left(\Delta_{c}, \mathbb{C}^{2}\right)$. Notice that $n_{ \pm}\left(D_{c}\right)=2$. The adjoint operator $D_{c}^{*}$ is given by

$$
D_{c}^{*} f=\left(-i c \frac{d}{d x} \otimes \sigma_{1}\right) f+\left(\frac{c^{2}}{2} \otimes \sigma_{3}\right) f, \quad f \in \operatorname{dom}\left(D_{c}^{*}\right)=W^{1,2}\left(\Delta_{c}, \mathbb{C}^{2}\right) .
$$

The triplet $\Pi_{D_{c}}=\left\{\mathcal{H}^{D_{c}}, \Gamma_{0}^{D_{c}}, \Gamma_{1}^{D_{c}}\right\}, \mathcal{H}^{D_{c}}:=\mathbb{C}^{2}$,

$$
\begin{aligned}
& \Gamma_{0}^{D_{c}}\left(\begin{array}{l}
f_{1} \\
f_{2}
\end{array}\right):=\frac{1}{\sqrt{2}}\left(\begin{array}{l}
f_{1}(a)+f_{1}(b) \\
f_{1}(a)-f_{1}(b)
\end{array}\right), \\
& \Gamma_{1}^{D_{c}}\left(\begin{array}{l}
f_{1} \\
f_{2}
\end{array}\right):=\frac{i c}{\sqrt{2}}\left(\begin{array}{l}
f_{2}(a)-f_{2}(b) \\
f_{2}(a)+f_{2}(b)
\end{array}\right),
\end{aligned}
$$

$f \in \operatorname{dom}\left(D_{c}^{*}\right)$, forms a boundary triplet for $D_{c}^{*}$. The $\gamma$-field and the Weyl function are given by

$$
\gamma^{D_{c}}(z)\left(\begin{array}{l}
\xi_{1} \\
\xi_{2}
\end{array}\right)=\frac{1}{\sqrt{2}}\left(\begin{array}{cc}
\frac{\cos (k(z)(x-\nu))}{\cos (k(z) d)} & \frac{\sin (k(z)(x-\nu))}{\sin (k(z) d)} \\
i k_{1}(z) \frac{\sin (k(z)(x-\nu))}{\cos (k(z) d} & i k_{1}(z) \frac{\cos (k(z)(x-\nu))}{\sin (k(z) d)}
\end{array}\right)\left(\begin{array}{l}
\xi_{1} \\
\xi_{2}
\end{array}\right),
$$

$z \in \mathbb{C}_{ \pm}$, and

$$
M^{D_{c}}(z)=\left(\begin{array}{cc}
m_{1}^{D_{c}}(z) & 0 \\
0 & m_{2}^{D_{c}}(z)
\end{array}\right), \quad z \in \mathbb{C}_{ \pm}
$$

where

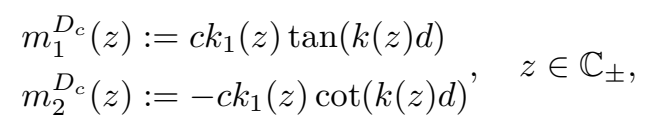


and $d:=\frac{b-a}{2}, \nu:=\frac{b+a}{2}$. Notice that the Weyl function $M^{D_{c}}(\cdot)$ is of quasi scalar type. The self-adjoint extension $D_{c}^{(1)}:=D_{c}^{*} \uparrow \operatorname{ker}\left(\Gamma_{0}^{D_{c}}\right)$ has the domain

$$
\operatorname{dom}\left(D_{c}^{(1)}\right)=\left\{f \in W^{1,2}\left(\Delta_{c}, \mathbb{C}^{2}\right): f_{1}(a)=f_{1}(b)=0\right\}
$$

while the extension $D_{c}^{(2)}:=D_{c}^{*}\left\lceil\operatorname{ker}\left(\Gamma_{1}^{D_{c}}\right)\right.$ has the domain

$$
\operatorname{dom}\left(D_{c}^{(2)}\right)=\left\{f \in W^{1,2}\left(\Delta_{c}, \mathbb{C}^{2}\right): f_{2}(a)=f_{2}(b)=0\right\} .
$$

We consider the closed symmetric operator

$$
S_{c}:=\overline{D_{c} \otimes I_{\mathfrak{T}}+I_{\mathfrak{D}_{c}} \otimes T}
$$

which is defined on $\mathfrak{K}_{c}:=\mathfrak{D}_{c} \otimes \mathfrak{T}=L^{2}\left(\Delta_{c},(\mathfrak{T} \oplus \mathfrak{T})^{t}\right)$. In the following we denote elements of of $\mathfrak{K}_{c}$ by $\vec{f}$. In particular, we use the notation

$$
\vec{f}=\left(\begin{array}{c}
\overrightarrow{f_{1}} \\
\overrightarrow{f_{2}}
\end{array}\right), \quad \overrightarrow{f_{j}} \in L^{2}\left(\Delta_{c}, \mathfrak{T}\right), \quad j=1,2 .
$$

Let us construct the boundary triplet $\Pi_{S_{c}}=\left\{\mathcal{H}^{S_{c}}, \Gamma_{0}^{S_{c}}, \Gamma_{1}^{S_{c}}\right\}$ for $S_{c}^{*}$. Since the Weyl function $M^{D_{c}}(\cdot)$ is of quasi scalar type we follow Remark 4.10, To this end we introduce the subspaces $\mathcal{H}_{1}^{D_{c}}:=\mathbb{C}$ and $\mathcal{H}_{2}^{D_{c}}=\mathbb{C}$. This yields $\mathcal{H}_{1}^{S_{c}}=\mathfrak{T}$ and $\mathcal{H}_{2}^{S_{c}}=\mathfrak{T}$ as well as

$$
\begin{aligned}
& \mathcal{H}^{S_{c}}=\mathcal{H}_{1}^{S_{c}}=\stackrel{\mathfrak{T}}{\oplus}=\stackrel{\oplus}{ } . \\
& \mathcal{H}_{2}^{S_{c}} \quad \mathfrak{T}
\end{aligned}
$$

Furthermore, we have

$$
\Gamma_{0}^{S_{c}} \vec{f}=\frac{1}{\sqrt{2}}\left(\begin{array}{l}
\sqrt{\operatorname{Im}\left(m_{1}^{D_{c}}(i-T)\right)}\left(\vec{f}_{1}(a)+\vec{f}_{1}(b)\right) \\
\sqrt{\operatorname{Im}\left(m_{2}^{D_{c}}(i-T)\right)}\left(\vec{f}_{1}(a)-\vec{f}_{1}(b)\right)
\end{array}\right)
$$

and

$$
\begin{aligned}
& \Gamma_{1}^{S_{c}} \vec{f}=
\end{aligned}
$$

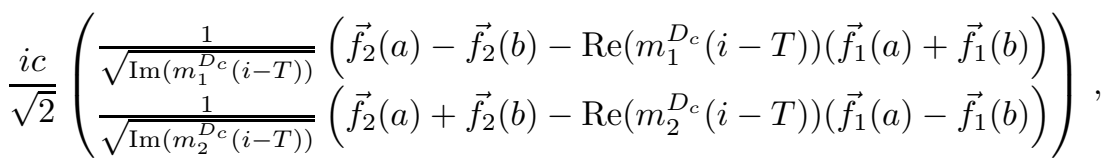

$\vec{f} \in \operatorname{dom}\left(D_{c}^{*} \otimes I_{\mathfrak{T}}\right) \cap \operatorname{dom}\left(I_{\mathfrak{D}_{c}} \otimes T\right)$. The $\gamma$-field $\gamma^{S_{c}}(\cdot): \mathcal{H}^{S_{c}} \longrightarrow \mathfrak{K}_{c}$ is computed by

$$
\begin{aligned}
& \left(\gamma^{S_{c}}(z) \vec{\xi}\right)(x)= \\
& \quad \frac{1}{\sqrt{2}}\left(\begin{array}{cc}
\frac{\cos (k(z-T)(x-\nu))}{\cos (k(z-T) d) \sqrt{\operatorname{Im}\left(m_{1}^{D_{c}}(i-T)\right)}} & -\frac{\sin (k(z-T)(x-\nu))}{\sin (k(z-T) d) \sqrt{\operatorname{Im}\left(m_{2}^{D_{c}}(i-T)\right)}} \\
i \frac{k_{1}(z-T) \sin (k(z-T)(x-\nu))}{\cos (k(z-T) d) \sqrt{\operatorname{Im}\left(m_{1}^{D_{c}}(i-T)\right)}} & i \frac{k_{1}(z-T) \cos (k(z-T)(x-\nu))}{\sin (k(z-T) d) \sqrt{\operatorname{Im}\left(m_{2}^{D_{c}}(i-T)\right)}}
\end{array}\right)\left(\begin{array}{l}
\xi_{1} \\
\xi_{2}
\end{array}\right),
\end{aligned}
$$


$z \in \mathbb{C}_{ \pm}$. The Weyl function $M^{S_{c}}(\cdot): \mathcal{H}^{S_{c}} \longrightarrow \mathcal{H}^{S_{c}}$ is given by

$$
M^{S_{c}}(z)=\left(\begin{array}{cc}
\frac{m_{1}^{D_{c}}(z-T)-\operatorname{Re}\left(m_{1}^{D_{c}}(i-T)\right)}{\operatorname{Im}\left(m_{1}^{D_{c}}(i-T)\right)} & 0 \\
0 & \frac{m_{2}^{D_{c}}(z-T)-\operatorname{Re}\left(m_{2}^{D_{c}}(i-T)\right)}{\operatorname{Im}\left(m_{2}^{D_{c}}(i-T)\right)}
\end{array}\right), \quad z \in \mathbb{C}_{ \pm} .
$$

Remark 6.2 Let us note that for the Dirac operator $D_{c}$ on a bounded interval the boundary triplet $\Pi_{D_{c}} \widehat{\otimes} I_{\mathfrak{T}}=\left\{\widehat{\mathcal{H}}^{S}, \widehat{\Gamma}_{0}^{S}, \widehat{\Gamma}_{1}^{S}\right\}$ where $\widehat{\mathcal{H}}^{S}:=\mathbb{C}^{2} \otimes \mathfrak{T}, \widehat{\Gamma}_{0}^{S}:=$ $\Gamma_{0}^{D_{c}} \widehat{\otimes} I_{\mathfrak{T}}$ and $\widehat{\Gamma}_{1}^{S}:=\Gamma_{1}^{D_{c}} \widehat{\otimes} I_{\mathfrak{T}}$ is already a boundary triplet for $S_{c}^{*}$ where $S_{c}=$ $D_{c} \otimes I_{\mathfrak{T}}+I_{\mathfrak{D}_{c}} \otimes T$, see [13]. In other words it is not necessary to regularize the boundary triplet $\Pi_{D_{c}} \widehat{\otimes} I_{\mathfrak{T}}$.

\section{A model for electronic transport through a boson cavity}

Let us propose a simple model describing the electronic transport through an optical cavity, cf. [52, 53. We consider the Hilbert space $\mathfrak{H}=L^{2}\left(\mathbb{R}_{-}\right) \oplus L^{2}\left(\mathbb{R}_{+}\right)$ where $\mathbb{R}_{-}=(-\infty, 0)$ and $\mathbb{R}_{+}=(0, \infty)$. On the subspaces $\mathfrak{H}_{l}:=L^{2}\left(\mathbb{R}_{-}\right)$and $\mathfrak{H}_{r}:=L^{2}\left(\mathbb{R}_{+}\right)$we consider the closed symmetric operators $H_{l}=-\frac{d^{2}}{d x^{2}}+v_{l}$ and $H_{r}=-\frac{d^{2}}{d x^{2}}+v_{r}$ of Subsection 6.1.1. We set $\mathfrak{H}:=\mathfrak{H}_{l} \oplus \mathfrak{H}_{r}=L^{2}(\mathbb{R})$ and $H:=H_{l} \oplus H_{r}$. Notice that $H$ can be regarded as the symmetric operator

$$
A=-\frac{d^{2}}{d x^{2}}+v(x), \quad v(x):= \begin{cases}v_{l} & x \in \mathbb{R}_{-} \\ v_{r} & x \in \mathbb{R}_{+}\end{cases}
$$

with domain $\operatorname{dom}(A)=W_{0}^{2,2}(\mathbb{R}):=\left\{f \in W^{2,2}(\mathbb{R}): f(0)=f^{\prime}(0)=0\right\}$. The operator $A$ is symmetric and has deficiency indices $n_{ \pm}(A)=2$. For simplicity we assume that

$$
0 \leq v_{r} \leq v_{l}
$$

Another one is the extension $A^{D}=H_{l}^{D} \oplus H_{r}^{D}$ where $H_{l}^{D}$ and $H_{r}^{D}$ are the extensions of $H_{l}$ and $H_{r}$, respectively, with Dirichlet boundary conditions at zero. One easily checks that the triple $\Pi_{A}=\left\{\mathcal{H}^{A}, \Gamma_{0}^{A}, \Gamma_{1}^{A}\right\}$ with

$$
\mathcal{H}^{A}:=\underset{\mathcal{H}^{H_{l}}}{\oplus}=\underset{\mathcal{H}^{H_{r}}}{\stackrel{\mathbb{C}}{\mathbb{C}}} \quad \underset{\mathbb{C}}{\mathbb{C}} \quad \Gamma_{0}^{A} f:=\left(\begin{array}{c}
f(-0) \\
f(+0)
\end{array}\right), \quad \Gamma_{1}^{A}:=\left(\begin{array}{c}
-f^{\prime}(-0) \\
f^{\prime}(+0)
\end{array}\right),
$$

defines a boundary triplet for $A^{*}$, cf. Subsection 6.1.1. The Weyl function $M^{A}(z)$ of the boundary triplet $\Pi_{A}$ is given by

$$
M^{A}(z)=\left(\begin{array}{cc}
m^{H_{l}}(z) & 0 \\
0 & m^{H_{r}}(z)
\end{array}\right)=\left(\begin{array}{cc}
i \sqrt{z-v_{l}} & 0 \\
0 & i \sqrt{z-v_{r}}
\end{array}\right), \quad z \in \rho\left(H^{D}\right) .
$$


where $A^{D}=A_{0}:=A^{*}\left\lceil\operatorname{ker}\left(\Gamma_{0}^{A}\right)\right.$.

Any other self-adjoint extension of $A$ is given by a self-adjoint relation $\Theta=$ $G(B)$ in $\mathbb{C}^{2}$, where $B$ is a self-adjoint operator given by

$$
B=\left(\begin{array}{ll}
\alpha & \gamma \\
\bar{\gamma} & \beta
\end{array}\right)
$$

cf. Proposition 2.3. The self-adjoint extension $A_{B}:=A^{*} \uparrow \operatorname{ker}\left(\Gamma_{1}^{A}-B \Gamma_{0}^{A}\right)$ corresponds to the boundary conditions

$$
\begin{aligned}
& f^{\prime}(-0)=-\alpha f(-0)-\gamma f(+0) \\
& f^{\prime}(+0)=\bar{\gamma} f(-0)+\beta f(+0)
\end{aligned}, \quad f \in \operatorname{dom}\left(A^{*}\right)=W^{2,2}\left(\mathbb{R}_{-}\right) \oplus W^{2,2}\left(\mathbb{R}_{+}\right) .
$$

If the matrix $B$ is diagonal, then there is no coupling between the left and right quantum system, i.e. the operator $A_{B}$ decomposes into a direct sum of two self-adjoint operators acting on $\mathfrak{H}_{l}$ and $\mathfrak{H}_{r}$, respectively. If $\gamma \neq 0$, then in some sense the left and right system interact.

Let us view the point zero as a quantum dot or quantum cavity. In particular, the Hilbert space $\mathcal{H}^{A}=\mathbb{C}^{2}$ is viewed as the state space of the quantum dot and the self-adjoint operator $B$ as the Hamiltonian of the dot. The Hamiltonian $B$ describes a two level system to which we are going to couple to bosons. The state space of the bosons is the Hilbert space $\mathfrak{T}=l_{2}\left(\mathbb{N}_{0}\right), \mathbb{N}_{0}:=\{0,1,2 \ldots\}$. The boson operator $T$ is given by

$$
\begin{aligned}
T \vec{\xi} & =T\left\{\xi_{k}\right\}_{k \in \mathbb{N}_{0}}=\left\{k \xi_{k}\right\}_{k \in \mathbb{N}_{0}}, \\
\vec{\xi} & =\left\{\xi_{k}\right\}_{k \in \mathbb{N}_{0}} \in \operatorname{dom}(T):=\left\{\left\{\xi_{k}\right\}_{k \in \mathbb{N}_{0}} \in l_{2}\left(\mathbb{N}_{0}\right):\left\{k \xi_{k}\right\}_{k \in \mathbb{N}_{0}} \in l_{2}\left(\mathbb{N}_{0}\right)\right\} .
\end{aligned}
$$

The Hamiltonian $T$ describes a system of bosons which do not interact mutually. The number of bosons is not fixed and varies from zero to infinity. The Hilbert space $\mathfrak{T}$ has a natural basis given by $e_{k}=\left\{\delta_{k j}\right\}_{j \in \mathbb{N}_{0}}$. Let us introduce the creation and annihilation operator $b^{*}$ and $b$, respectively, defined by

$$
b^{*} e_{k}=\sqrt{k+1} e_{k+1}, \quad k \in \mathbb{N}_{0}, \quad \text { and } \quad b e_{k}=\sqrt{k} e_{k-1}, \quad k \in \mathbb{N}_{0},
$$

where $e_{-1}=0$. One easily checks that $T=b^{*} b$.

Let us consider the compound system $\left\{\mathcal{H}^{C}, C\right\}$ consisting of the two level quantum system $\left\{\mathcal{H}^{A}, B\right\}$ and of the boson system $\left\{l_{2}\left(\mathbb{N}_{0}\right), T\right\}$. Its state space is given by $\mathcal{H}^{C}:=\mathcal{H}^{A} \otimes l_{2}\left(\mathbb{N}_{0}\right)=l_{2}\left(\mathbb{N}_{0}, \mathcal{H}^{A}\right)=l_{2}\left(\mathbb{N}_{0}, \mathbb{C}^{2}\right)$ and the compound Hamiltonian $C:=\overline{B \otimes I_{\mathfrak{T}}+I_{\mathcal{H}^{A}} \otimes T}$ where obviously $\operatorname{dom}(C)=\operatorname{dom}\left(I_{\mathcal{H}^{A}} \otimes\right.$ $T)$. The Hamiltonian $C$ does not describe any interaction between the system $\left\{\mathcal{H}^{A}, B\right\}$ and the bosons. To introduce such an interaction we consider the so-called Jaynes-Cumming Hamiltonian, cf. 37. To this end we consider the eigenvalues $\lambda_{0}^{B}$ and $\lambda_{1}^{B}$ of $\mathrm{B}$ and the corresponding normalized eigenvectors $e_{0}^{B}$ and $e_{1}^{B}$. We assume that $\lambda_{0}^{B}<\lambda_{1}^{B}$. Notice that $B$ admits the representation

$$
B=\lambda_{0}^{B}\left(\cdot, e_{0}^{B}\right) e_{0}^{B}+\lambda_{1}^{B}\left(\cdot, e_{1}^{B}\right) e_{1}^{B} .
$$


Let us define the matrices

$$
\begin{aligned}
\sigma_{+}^{B} e_{0}^{B}=e_{1}^{B}, & \sigma_{+}^{B} e_{1}^{B}=0 \\
\sigma_{-}^{B} e_{0}^{B}=0, & \sigma_{-}^{B} e_{1}^{B}=e_{0}^{B} .
\end{aligned}
$$

One easily checks that

$$
B=\lambda_{1}^{B} \sigma_{+}^{B} \sigma_{-}^{B}+\lambda_{0}^{B} \sigma_{-}^{B} \sigma_{+}^{B} .
$$

We set

$$
V_{J C}:=\sigma_{+}^{B} \otimes b+\sigma_{-}^{B} \otimes b^{*}, \quad \operatorname{dom}\left(V_{J C}\right)=\operatorname{dom}\left(I_{\mathcal{H}^{A}} \otimes \sqrt{T}\right),
$$

and define the Jaynes-Cummings Hamiltonian $C_{J C}$ by setting

$$
C_{J C}=B \otimes I_{\mathfrak{T}}+I_{\mathcal{H}^{A}} \otimes T+\tau V_{J C}, \quad \tau \in \mathbb{R} .
$$

One easily checks that the perturbation $V_{J C}$ is infinitesimally small with respect to $I_{\mathcal{H}^{A}} \otimes T$ which yields that $C_{J C}$ is self-adjoint with domain $\operatorname{dom}\left(C_{J C}\right)=$ $\operatorname{dom}\left(I_{\mathcal{H}^{A}} \otimes T\right)$.

Let us consider the closed symmetric operator

$$
S:=A \otimes I_{\mathfrak{T}}+I_{\mathfrak{H}} \otimes T
$$

in the Hilbert space $\mathfrak{K}:=\mathfrak{H} \otimes \mathfrak{T}$. Setting

$$
\begin{array}{ll}
\mathfrak{K}_{l}:=\mathfrak{H}_{l} \otimes \mathfrak{T}, & S_{l}:=H_{l} \otimes I_{\mathfrak{T}}+I_{\mathfrak{H}_{l}} \otimes T, \\
\mathfrak{K}_{r}:=\mathfrak{H}_{r} \otimes \mathfrak{T}, & S_{r}:=H_{r} \otimes I_{\mathfrak{T}}+I_{\mathfrak{H}_{r}} \otimes T,
\end{array}
$$

we obtain

$$
\mathfrak{K}=\mathfrak{K}_{l} \oplus \mathfrak{K}_{r} \quad \text { and } \quad S=S_{l} \oplus S_{r}
$$

It is desirable to define the operator $\widetilde{S}=\widetilde{S}^{*}$ describing the point contact of the quantum system $\left\{\mathfrak{H}, A_{B}\right\}$ with $A_{B}:=A^{*}\left\lceil\operatorname{ker}\left(\Gamma_{1}^{A}-B \Gamma_{0}^{A}\right)\right.$, to the boson reservoir treating $C_{J C}$ as the boundary operator with respect to a triplet $\Pi_{S}=$ $\Pi_{A} \widehat{\otimes} I_{\mathfrak{T}}$, i.e. by setting $\widetilde{S}:=S_{C_{J C}}$. However, the triplet $\Pi_{S}=\Pi_{A} \widehat{\otimes} I_{\mathfrak{T}}=$ $\Pi_{H_{l}} \widehat{\otimes} I_{\mathfrak{T}} \oplus \Pi_{H_{r}} \widehat{\otimes} I_{\mathfrak{T}}$ is in general not a boundary triplet for $S^{*}$ if $T$ is unbounded. Therefore the above treatment is incorrect. To get a boundary triplet for $S^{*}$ one has to regularize the triplet $\Pi_{S}$ in accordance with Theorem 4.8, This leads to the boundary triplet $\widetilde{\Pi}_{S}=\left\{\mathcal{H}^{S}, \widetilde{\Gamma}_{0}^{S}, \Gamma_{1}^{S}\right\}=\Pi_{S_{l}} \oplus \Pi_{S_{r}}$, where $\Pi_{S_{r}}$ and $\Pi_{S_{l}}$ are the normalized boundary triplets (6.3) and (6.6), respectively. The corresponding Weyl function $M^{S}(\cdot)$ is given by

$$
M^{S}(z)=\left(\begin{array}{cc}
M^{S_{l}}(z) & 0 \\
0 & M^{S_{r}}(z)
\end{array}\right), \quad z \in \rho\left(S_{0}\right), \quad S_{0}=S_{l}^{D} \oplus S_{r}^{D},
$$

where $M^{S_{r}}(\cdot)$ and $M^{S_{l}}(\cdot)$ are defined by (6.4) and (6.7), respectively. Moreover, $S_{l}^{D}=H_{l}^{D} \otimes I_{\mathfrak{T}}+I_{\mathfrak{H}_{l}} \otimes T$ and $S_{r}^{D}=H_{r}^{D} \otimes I_{\mathfrak{T}}+I_{\mathfrak{H}_{l}} \otimes T$. 
The boundary operator $\widetilde{C}_{J C}$ corresponding to the extension $\widetilde{S}:=S_{C_{J C}}$ in the boundary triplet $\widetilde{\Pi}_{S}$ is the following regularization of $C_{J C}$ :

$$
\widetilde{C}_{J C}:=R^{-1}\left(C_{J C}-Q\right) R^{-1}, \quad \operatorname{dom}\left(\widetilde{C}_{J C}\right)=\operatorname{dom}\left(T^{3 / 2}\right),
$$

with

$$
Q:=\left(\begin{array}{cc}
Q_{l} & 0 \\
0 & Q_{r}
\end{array}\right)=\left(\begin{array}{cc}
\operatorname{Re}\left(m^{H_{l}}(i-T)\right) & 0 \\
0 & \operatorname{Re}\left(m^{H_{r}}(i-T)\right)
\end{array}\right)
$$

and

$$
R:=\left(\begin{array}{cc}
R_{l} & 0 \\
0 & R_{r}
\end{array}\right)=\left(\begin{array}{cc}
\sqrt{\operatorname{Im}\left(m^{H_{l}}(i-T)\right)} & 0 \\
0 & \sqrt{\operatorname{Im}\left(m^{H_{r}}(i-T)\right)}
\end{array}\right),
$$

where $m^{H_{r}}(\cdot)$ and $m^{H_{l}}(\cdot)$ are given by (6.1) and (6.5).

Next we show that the operator $\widetilde{C}_{J C}$ is well-defined and self-adjoint. We set

$$
\widetilde{C}:=R^{-1}(C-Q) R^{-1} \quad \text { and } \quad \widetilde{V}_{J C}:=R^{-1} V_{J C} R^{-1}
$$

and consider the representation $\widetilde{C}_{J C}=\widetilde{B}+\widetilde{T}+\tau \widetilde{V}_{J C}=\widetilde{C}+\tau \widetilde{V}_{J C}$ where

$$
\widetilde{C}:=\widetilde{B}+\widetilde{T} \quad \text { and } \quad \widetilde{B}:=R^{-1}\left(B \otimes I_{\mathfrak{T}}\right) R^{-1}, \quad \widetilde{T}:=R^{-1}\left(I_{\mathcal{H}^{A}} \otimes T-Q\right) R^{-1} .
$$

It follows with account of (17.3) and (17.4) that

$$
\begin{aligned}
\widetilde{T}:=R^{-1} & \left(I_{\mathcal{H}^{A}} \otimes T-Q\right) R^{-1} \\
& =\left(\begin{array}{cc}
R_{l}^{-1}\left(T-Q_{l}\right) R_{l}^{-1} & 0 \\
0 & R_{r}^{-1}\left(T-Q_{r}\right) R_{r}^{-1}
\end{array}\right)=:\left(\begin{array}{cc}
\widetilde{T}_{l} & 0 \\
0 & \widetilde{T}_{r}
\end{array}\right) .
\end{aligned}
$$

Let us introduce the operators

$$
\begin{aligned}
& Z_{l}:=\sqrt{\sqrt{I_{\mathfrak{T}}+\left(T+v_{l}\right)^{2}}+T+v_{l}} \geq I_{\mathfrak{T}}, \\
& Z_{r}:=\sqrt{\sqrt{I_{\mathfrak{T}}+\left(T+v_{r}\right)^{2}}+T+v_{r}} \geq I_{\mathfrak{T}} .
\end{aligned}
$$

Clearly, these operators are self-adjoint and $\operatorname{dom}\left(Z_{l}\right)=\operatorname{dom}\left(Z_{r}\right)=\operatorname{dom}\left(T^{1 / 2}\right)$. A straightforward computation shows that

$$
R=\frac{1}{\sqrt[4]{2}}\left(\begin{array}{cc}
Z_{l}^{-1 / 2} & 0 \\
0 & Z_{r}^{-1 / 2}
\end{array}\right)
$$

and

$$
Q=-\frac{1}{\sqrt{2}}\left(\begin{array}{cc}
Z_{l} & 0 \\
0 & Z_{r}
\end{array}\right)
$$

It follows that

$$
\widetilde{T}=\left(\begin{array}{cc}
\sqrt{2} T Z_{l}+Z_{l}^{2} & 0 \\
0 & \sqrt{2} T Z_{r}+Z_{r}^{2}
\end{array}\right)
$$


It is easily seen that $\operatorname{dom}(\widetilde{T})=\operatorname{dom}\left(T^{3 / 2}\right) \oplus \operatorname{dom}\left(T^{3 / 2}\right)$ and $\widetilde{T}=\widetilde{T}^{*}$. Moreover, it satisfies $\widetilde{T} \geq I_{\mathcal{H}^{A} \otimes \mathfrak{T}}$. The operator $\widetilde{B}=R^{-1}\left(B \otimes I_{\mathfrak{T}}\right) R^{-1}$ takes the form

$$
\widetilde{B}=\sqrt{2}\left(\begin{array}{cc}
\alpha Z_{l} & \gamma Z_{l}^{1 / 2} Z_{r}^{1 / 2} \\
\gamma Z_{r}^{1 / 2} Z_{l}^{1 / 2} & \beta Z_{r}
\end{array}\right) .
$$

This operator is symmetric on the natural domain $\operatorname{dom}\left(T^{1 / 2}\right) \oplus \operatorname{dom}\left(T^{1 / 2}\right)$. Moreover, one easily checks that $\widetilde{B}$ is infinitesimally small with respect to $\widetilde{T}$. Hence the sum $\widetilde{C}:=\widetilde{T}+\widetilde{B}$ is self-adjoint on the domain $\operatorname{dom}\left(T^{3 / 2}\right) \oplus \operatorname{dom}\left(T^{3 / 2}\right)$. Furthermore, a straightforward computation shows that the operator $\widetilde{V}_{J C}$ is symmetric on $\operatorname{dom}(T)$. Moreover, $\widetilde{V}_{J C}$ is infinitesimally small with respect to $\widetilde{T}$. Therefore the operator sum $\widetilde{C}_{J C}=\widetilde{B}+\widetilde{T}+\widetilde{V}_{J C}$ is well-defined on $\operatorname{dom}\left(T^{3 / 2}\right) \oplus \operatorname{dom}\left(T^{3 / 2}\right)$ and self-adjoint.

According to (4.27) the boundary triplet $\widetilde{\Pi}_{S}:=\left\{\mathcal{H}^{S}, \widetilde{\Gamma}_{0}^{S}, \widetilde{\Gamma}_{1}^{S}\right\}$ takes the form

$$
\widetilde{\Gamma}_{0}^{S} f=R\left(\Gamma_{0}^{H} \widehat{\otimes} I_{\mathfrak{T}}\right) f, \quad \text { and } \quad \widetilde{\Gamma}_{1}^{S} f=R^{-1}\left(\Gamma_{1}^{H} \widehat{\otimes} I_{\mathfrak{T}}-Q \Gamma_{0}^{H} \widehat{\otimes} I_{\mathfrak{T}}\right) f
$$

for $f \in \mathfrak{D}=\operatorname{dom}\left(S^{*}\right) \cap \operatorname{dom}\left(I_{\mathfrak{H}} \otimes T\right)$. Hence the Hamiltonian $\widetilde{S}$ describing the contact to the reservoir is given by

$$
\begin{aligned}
\widetilde{S}=S_{\widetilde{C}_{J C}} & :=S^{*}\left\lceil\operatorname{dom}\left(S_{\widetilde{C}_{J C}}\right),\right. \\
\operatorname{dom}\left(S_{\widetilde{C}_{J C}}\right) & :=\left\{f \in \operatorname{dom}\left(S^{*}\right): \widetilde{\Gamma}_{1}^{S} f=\widetilde{C}_{J C} \widetilde{\Gamma}_{0}^{S} f\right\} .
\end{aligned}
$$

Inserting (7.5) into (7.6) one rewrites the last relation as

$$
\operatorname{dom}\left(S_{\widetilde{C}_{J C}}\right) \cap \mathfrak{D}=\left\{f \in \mathfrak{D}: \Gamma_{1}^{H} \widehat{\otimes} I_{\mathfrak{T}} f=C_{J C} \Gamma_{0}^{H} \widehat{\otimes} I_{\mathfrak{T}} f\right\}
$$

which coincides with the original boundary condition.

The system $\left\{\mathfrak{K}, S_{\widetilde{C}_{J C}}\right\}$ can be regarded as the Jaynes-Cummings model coupled to leads. It describes the electronic transport through a dot or cavity where the electrons interact with bosons. Notice that the interaction of electrons to bosons is restricted only to one point.

Remark 7.1 Let us make some comments.

(i) The system $\left\{\mathfrak{K}, S_{0}\right\}, S_{0}:=S^{*}\left\lceil\operatorname{ker}\left(\Gamma_{0}^{S}\right)=A^{D} \otimes I_{\mathfrak{T}}+I_{\mathfrak{H}_{H}} \otimes T\right.$ describes a situation where the left system $\left\{\mathfrak{K}_{l}, S_{l}^{D}\right\}, S_{l}^{D}:=S_{l}^{*} \uparrow \operatorname{ker}\left(\Gamma_{0}^{S_{l}}\right)$, and right system $\left\{\mathfrak{K}_{r}, S_{r}^{D}\right\}, S_{r}^{D}:=S_{r}^{*} \uparrow \operatorname{ker}\left(\Gamma_{0}^{S_{r}}\right)$ are completely decoupled.

(ii) If $\tau=0$, then $\widetilde{C}_{J C}=\widetilde{C}$. The system $\left\{\mathfrak{K}, S_{\widetilde{C}}\right\}$ describes a situation where the left and right systems $\left\{\mathfrak{K}_{l}, S_{l}^{D}\right\}$ and $\left\{\mathfrak{K}_{r}, S_{r}^{D}\right\}$ are coupled by a point interaction at zero but not by a boson-electron interaction. In particular, if $B$ is diagonal, then again the left and right systems $\left\{\mathfrak{K}_{l}, S_{l}^{D}\right\}$ and $\left\{\mathfrak{K}_{r}, S_{r}^{D}\right\}$ are decoupled.

(iii) If $\tau \neq 0$, then the system $\left\{\mathfrak{K}, S_{\widetilde{C}_{J C}}\right\}$ can be viewed as fully coupled: the left and right systems are coupled by point interaction at zero as well as by a boson-electron interaction at zero. 
(iv) If $\tau \neq 0$ and $B$ is diagonal, then the left and right systems are only coupled by the boson-electron interaction at zero.

(v) The model above can be viewed as a simple model of a solar cell or a light emitting diode (LED).

(vi) Using the result of subsection 6.2.1 one can introduce a similar model where the Schrödinger operators $H_{l}$ and $H_{r}$ are replaced by Dirac operators $D_{l}$ and $D_{r}$, respectively.

(vii) Finally, we mention that the self-adjoint operators $C_{J C}$ and $\widetilde{C}_{J C}$ have Jacobi structure in a basis formed by the orthogonal systems $\left\{e_{0}^{B} \otimes e_{k}, e_{1}^{B} \otimes\right.$ $\left.e_{k}\right\}_{k \in \mathbb{N}_{0}}$ in $\mathcal{H}^{A} \otimes \mathfrak{T}$. To see this we note first that the subspaces spanned by $\left\{e_{0}^{B} \otimes e_{k+1}\right\}_{k \in \mathbb{N}_{0}}$ and $\left\{e_{1}^{B} \otimes e_{k}\right\}_{k \in \mathbb{N}_{0}}$, respectively, leave invariant the self-adjoint operators $T+\tau V_{J C}$ and $\widetilde{T}+\tau \widetilde{V}_{J C}$. Secondary, the operators $B \otimes I_{\mathfrak{T}}$ and $\widetilde{B}$ leave invariant the subspace spanned by the systems $\left\{e_{0}^{B} \otimes e_{k}\right\}_{k \in \mathbb{N}_{0}}$ and $\left\{e_{1}^{B} \otimes e_{k}\right\}_{k \in \mathbb{N}_{0}}$, respectively. Both statements ensures the Jacobi structure of both operators $C_{J C}$ and $\widetilde{C}_{J C}$.

\section{Acknowledgments}

This work was financially supported by the Ministry of Education and Science of the Russian Federation (the Agreement number No. 02.A03.21.0008), by the Government of the Russian Federation (grant 074-U01), by grant MK-5161.2016.1 of the President of the Russian Federation, by DFG grants NE 1439/3-1 and BR 1686/3-1, and by grant 16-11-10330 of the Russian Science Foundation. The preparation of the paper was supported by the European Research Council via ERC-2010-AdG no 267802 ("Analysis of Multiscale Systems Driven by Functionals"). I. P. and A. B. thank WIAS (Berlin) for hospitality and I. P. also TU Clausthal. H. N. thanks the ITMO University of St. Petersburg (Russia) for financial support and hospitality.

\section{References}

[1] N.R. Abdullah, Chi-S. Tang, A. Manolescu, and V. Gudmundsson. Electron transport through a quantum dot assisted by cavity photons. Journal of Physics: Condensed Matter, 25(46):465302, 2013.

[2] N.I. Achieser and I.M. Glasmann. Theorie der linearen Operatoren im Hilbert-Raum. Verlag Harri Deutsch, Thun, eighth edition, 1981.

[3] V. Adamyan, H. Langer, R. Mennicken, and J. Saurer. Spectral components of self-adjoint block operator matrices with unbounded entries. Math. Nachr., 178:43-80, 1996. 
[4] S. Albeverio, J.F. Brasche, M.M. Malamud, and H. Neidhardt. Inverse spectral theory for symmetric operators with several gaps: scalar-type Weyl functions. J. Funct. Anal., 228(1):144-188, 2005.

[5] S. Albeverio, F. Gesztesy, R. Høegh-Krohn, and H. Holden. Solvable models in quantum mechanics. AMS Chelsea Publishing, Providence, RI, 2005.

[6] W. Aschbacher, V. Jaksic, Y. Pautrat, and C.-A. Pillet. Transport properties of quasi-free fermions. J. Math. Phys., 48:032101, 2007.

[7] H. Baumgärtel and M. Wollenberg. Mathematical scattering theory. Akademie-Verlag, Berlin, 1983.

[8] J. Behrndt, M. Langer, V. Lotoreichik. Schrödinger operators with $\delta$ and $\delta^{\prime}$-potentials supported on hypersurfaces. Ann. Henri Poincaré 14: $385-$ $423,2013$.

[9] J. Behrndt, M. Langer, I. Lobanov, V. Lotoreichik, I.Yu. Popov. A remark on Schatten-von Neumann properties of resolvent differences of generalized Laplacians on bounded domains. J. Math. Anal. Appl. 371: 750-758, 2010.

[10] J. Behrndt, M.M. Malamud, and H. Neidhardt. Scattering matrices and Weyl functions. Proc. Lond. Math. Soc. (3), 97, 2008.

[11] J. Behrndt, M.M. Malamud, and H. Neidhardt. Scattering matrices and Dirichlet-to-Neumann maps. J. Funct. Anal., 273(6):1970-2025, 2017.

[12] M.Š. Birman and M.Z. Solomjak. Spectral theory of self-adjoint operators in Hilbert space. D. Reidel Publishing Co., Dordrecht, 1987.

[13] A.A. Boitsev, H. Neidhardt, and I.Y. Popov. Dirac operator coupled to bosons. Nanosyst., Phys. Chem. Math., 7(2):332-339, 2016.

[14] A.A. Boitsev, H. Neidhardt, and I.Yu. Popov. Weyl function for sum of operators tensor products. Nanosystems: Physics, Chemistry, Mathematics, 4(6):747-757, 2013.

[15] J.F. Brasche, M.M. Malamud, and H. Neidhardt. Weyl function and spectral properties of self-adjoint extensions. Integral Equations Operator Theory, 43(3):264-289, 2002.

[16] B.M. Brown, G. Grubb, I. Wood, M-functions for closed extensions of adjoint pairs of operators with applications to elliptic boundary problems. Math. Nachr., 282: 314-347, 2009.

[17] B.M. Brown, M. Marletta, S. Naboko, I. Wood, Boundary triplets and Mfunctions for non-self-adjoint operators, with applications to elliptic PDEs and block operator matrices. J. Lond. Math. Soc. (2) 77: 700-718, 2009. 
[18] J. Brüning, V. Geyler, and K. Pankrashkin. Spectra of self-adjoint extensions and applications to solvable Schrödinger operators. Rev. Math. Phys., 20(1):1-70, 2008.

[19] M. Büttiker, Y. Imry, R. Landauer, and S. Pinhas. Generalized manychannel conductance formula with application tosmall rings. Phys. Rev. B, 31(10):6207-6215, 1985.

[20] R. Carlone, M. Malamud, and A. Posilicano. On the spectral theory of Gesztesy-Šeba realizations of 1-D Dirac operators with point interactions on a discrete set. J. Differential Equations, 254(9):3835-3902, 2013.

[21] H.D. Cornean, H. Neidhardt, L. Wilhelm, and V.A. Zagrebnov. The Cayley transform applied to non-interacting quantum transport. J. Funct. Anal., 266(3):1421-1475, 2014.

[22] E.B. Davies. Quantum theory of open systems. Academic Press, LondonNew York, 1976.

[23] J. Dereziński and R. Früboes. Fermi golden rule and open quantum systems. In Open quantum systems. III, volume 1882 of Lecture Notes in Math., pages 67-116. Springer, Berlin, 2006.

[24] J. Dereziński and V. Jakšić. On the nature of Fermi golden rule for open quantum systems. J. Statist. Phys., 116(1-4):411-423, 2004.

[25] V. Derkach, S. Hassi, M. Malamud, and H. de Snoo. Boundary triplets and Weyl functions. Recent developments. In Operator methods for boundary value problems, volume 404 of London Math. Soc. Lecture Note Ser., pages 161-220. Cambridge Univ. Press, Cambridge, 2012.

[26] V.A. Derkach, S. Hassi, M.M. Malamud, and H.S.V. de Snoo. Boundary relations and their Weyl families. Trans. Amer. Math. Soc., 358(12):53515400 (electronic), 2006.

[27] V.A. Derkach and M.M. Malamud. The extension theory of Hermitian operators and the moment problem. J. Math. Sci., 73(2):141-242, 1995.

[28] V.A. Derkach and M.M. Malamud. Generalized resolvents and boundary value problems for Hermitian operators with gaps. J. Funct. Anal., 95(1):195, 1991.

[29] V.A. Derkach and M.M. Malamud. On the Weyl function and Hermite operators with lacunae. Dokl. Akad. Nauk SSSR, 293(5):1041-1046, 1987.

[30] J. Eckhardt, A. Kostenko, M. Malamud, and G. Teschl. One-dimensional Schrödinger operators with $\delta^{\prime}$-interactions on Cantor-type sets. J. Differential Equations, 257(2):415-449, 2014. 
[31] M.L. Gorbachuk. self-adjoint boundary problems for a second-order differential equation with unbounded operator coefficient. Funct. Anal. Appl., $5: 9-18,1971$.

[32] V.I. Gorbachuk and M.L. Gorbachuk. Boundary value problems for operator differential equations. Kluwer Academic Publishers Group, Dordrecht, 1991.

[33] G. Grubb, A characterization of the non local boundary value problems associated with an elliptic operator, Ann. Scuola Normale Superiore de Pisa, 22(3): 425-513, 1968.

[34] G. Grubb, Distributions and Operators, Vol 552, Grad. Texts in Math., Springer, New York, 2009.

[35] G. Grubb, The mixed boundary value problem, Kreın resolvent formula and spectral asymptotic estimates, J. Math. Anal. and Appl., 382: 339-363, 2011.

[36] Q. Hu. Photon-assisted quantum transport in quantum point contacts. Applied physics letters, 62(8):837-839, 1993.

[37] E.T. Jaynes and F.W. Cummings. Comparison of quantum and semiclassical radiation theories with application to the beam maser. Proceedings of the IEEE, 51(1):89-109, 1963.

[38] T. Kato. Perturbation theory for linear operators. Springer-Verlag, Berlin, second edition, 1976. Grundlehren der Mathematischen Wissenschaften, Band 132.

[39] A.N. Kočubeř. Symmetric operators and nonclassical spectral problems. Mat. Zametki, 25(3):425-434, 477, 1979.

[40] A. Kostenko and M. Malamud. 1-D Schrödinger operators with local point interactions: a review. Proc. Sympos. Pure Math., 87:235-262, 2013.

[41] A. Kostenko and M. Malamud. Spectral theory of semibounded Schrödinger operators with $\delta^{\prime}$-interactions. Ann. Henri Poincaré, 15(3):501-541, 2014.

[42] A.S. Kostenko and M.M. Malamud. 1-D Schrödinger operators with local point interactions on a discrete set. J. Differential Equations, 249(2):253304, 2010.

[43] A.S. Kostenko, M.M. Malamud, and D.D. Natyagaullo. The matrix Schrödinger operator with $\delta$-interactions. Mat. Zametki, 100(1):59-77, 2016.

[44] L.P. Kouwenhoven, S. Jauhar, K. McCormick, D. Dixon, P.L. McEuen, Yu.V. Nazarov, N.C. van der Vaart, and C.T. Foxon. Photon-assisted tunneling through a quantum dot. Phys. Rev. B, 50:2019-2022, 1994. 
[45] M.G. Krein. The theory of self-adjoint extensions of semi-bounded Hermitian transformations and its applications. I. Rec. Math. [Mat. Sbornik] N.S., 20(62):431-495, 1947.

[46] R. Landauer. Spatial Variation of Currents and Fields Due to Localized Scatterers in Metallic Conduction. IBM J. Res. Develop., 1(3):223-231, 1957.

[47] M.M. Malamud. Some classes of extensions of a Hermitian operator with lacunae. Ukraïn. Mat. Zh., 44(2):215-233, 1992.

[48] M.M. Malamud, Spectral theory of elliptic operators in exterior domains. Russ. J. Math. Phys. 17(1): 96-125, 2010.

[49] M.M. Malamud and H. Neidhardt. On the unitary equivalence of absolutely continuous parts of self-adjoint extensions. J. Funct. Anal., 260(3):613-638, 2011.

[50] M.M. Malamud and H. Neidhardt. Sturm-Liouville boundary value problems with operator potentials and unitary equivalence. J. Differential Equations, 252(11):5875-5922, 2012. (arXiv:0907.0650v1 [math-ph], 2009).

[51] M.M. Malamud and K. Schmüdgen. Spectral theory of Schrödinger operators with infinitely many point interactions and radial positive definite functions. J. Funct. Anal., 263(10):3144-3194, 2012.

[52] H. Neidhardt, L. Wilhelm, and V.A. Zagrebnov. A new model of quantum dot light emitting-absorbing devices. Zh. Mat. Fiz. Anal. Geom., 10(3):350$385,2014$.

[53] H. Neidhardt, L. Wilhelm, and V.A. Zagrebnov. A new model for quantum dot light emitting-absorbing devices: proofs and supplements. Nanosystems: Physics, Chemistry, Mathematics, 6(1):6-45, 2015.

[54] B.S. Pavlov. A model of zero-radius potential with internal structure. Teoret. Mat. Fiz., 59(3):345-353, 1984.

[55] B.S. Pavlov. The theory of extensions, and explicitly solvable models. Uspekhi Mat. Nauk, 42(6(258)):99-131, 247, 1987.

[56] B.S. Pavlov and A.A. Shushkov. The theory of extensions, and null-range potentials with internal structure. Mat. Sb. (N.S.), 137(179)(2):147-183, $271,1988$.

[57] M.H. Pedersen and M. Büttiker. Scattering theory of photon-assisted electron transport. Phys. Rev. B, 58:12993-13006, Nov 1998.

[58] K. Schmüdgen. Unbounded self-adjoint operators on Hilbert space, volume 265 of Graduate Texts in Mathematics. Springer, Dordrecht, 2012. 
[59] J. von Neumann. Allgemeine Eigenwerttheorie Hermitescher Funktionaloperatoren. Math. Ann., 102:49-131, 1929.

[60] K. Yosida. Functional analysis, volume 123 of Grundlehren der Mathematischen Wissenschaften. Springer-Verlag, Berlin-New York, sixth edition, 1980 .

\section{Addresses:}

A.A. Boitsev,

St. Petersburg National Research University of Information Technologies, Mechanics and Optics, 49 Kronverkskiy, St. Petersburg 197101, Russia

E-mail: boitsevanton@gmail.com

J.F. Brasche

Institut für Mathematik, TU Clausthal, Erzstr. 1, D-38678 Clausthal-Zellerfeld, Germany

E-mail:johannes.brasche@tu-clausthal.de

M.M. Malamud

Institute of Applied Mathematics and Mechanics, NAS Ukraine,

Peoples Friendship University of Russia (RUDN University), 6 MiklukhoMaklaya Street, Moscow, 117198, Russia

E-mail: malamud3m@gmail.com

H. Neidhardt, Weierstrass Institute of Applied Analysis and Stoachstics, Mohrenstr. 39, D-10117 Berlin, Germany

E-mail: hagen.neidhardt@wias-berlin.de

I.Yu. Popov

St. Petersburg National Research University of Information Technologies, Mechanics and Optics, 49 Kronverkskiy, St. Petersburg 197101, Russia

E-mail: popov1955@gmail.com 